\title{
The Effect of Attenuation from Fish on Long-Range Active and Passive Acoustic Sensing in the Ocean
}

\author{
by \\ Daniel Michael Duane \\ B.S., The College of William \& Mary (2015) \\ Submitted to the Department of Mechanical Engineering \\ and the Joint Program in Applied Ocean Science \& Engineering \\ in partial fulfillment of the requirements for the degree of \\ Doctor of Philosophy \\ at the \\ MASSACHUSETTS INSTITUTE OF TECHNOLOGY \\ and the \\ WOODS HOLE OCEANOGRAPHIC INSTITUTION \\ February 2022 \\ (C)2022 Daniel Duane. \\ All rights reserved.
}

The author hereby grants to MIT permission to reproduce and to distribute publicly

paper and electronic copies of this thesis document in whole or in part in any medium now known or hereafter created.

Author

Joint Program in Applied Ocean Science \& Engineering Massachusetts Institute of Technology \& Woods Hole Oceanographic Institution

October 5, 2021

Certified by

Nicholas Makris

Professor of Mechanical and Ocean Engineering Massachusetts Institute of Technology

Thesis Supervisor

Accepted by

Nicolas Hadjiconstantinou

Professor of Mechanical Engineering Chair, Department Committee on Graduate Students, Massachusetts Institute of Technology

Accepted by

David Ralston

Associate Scientist with Tenure, Applied Ocean Physics \& Engineering Department Chair, Joint Committee for Applied Ocean Science \& Engineering Woods Hole Oceanographic Institution 


\title{
The Effect of Attenuation from Fish on Long-Range Active and Passive Acoustic Sensing in the Ocean
}

\author{
by \\ Daniel Michael Duane \\ Submitted to the Joint Program in Applied Ocean Science \& Engineering
Massachusetts Institute of Technology
\& Woods Hole Oceanographic Institution
on October 5,2021 , in partial fulfillment of the
requirements for the degree of
Doctor of Philosophy
}

\begin{abstract}
Attenuation from fish can reduce the intensity of acoustic signals and significantly decrease detection range for long-range active and passive sensing in the ocean. This makes it important to understand the relevant mechanisms and accurately predict attenuation from fish in underwater acoustic sensing. Formulations for predicting attenuation from fish, however, depend on the accurate characterization of population density and spatial distribution of fish groups along long-range propagation paths, which is difficult to achieve using conventional survey methods. In previous investigations of attenuation from fish, population densities were inferred from reductions in the intensity of long-range acoustic signals caused by diel or seasonal shoaling patterns of fish groups. Here, Ocean Acoustic Waveguide Remote Sensing (OAWRS) is used to instantaneously image massive Norwegian herring shoals that stretch for thousands of square kilometers and simultaneously measure attenuation from these shoals within the active OAWRS transmissions, as well as attenuation to ship-radiated tonals detected by Passive Ocean Acoustic Waveguide Remote Sensing (POAWRS). Reductions in signal intensity are predicted using a normal-mode-based analytical theory derived from first principles for acoustic propagation and scattering through inhomogeneities in an ocean waveguide. The predictions of the waveguide attenuation formulation are in agreement with measured reductions from attenuation, where the position, size, and population density of the fish groups are characterized using OAWRS imagery as well as in situ echosounder measurements of the specific shoals occluding the propagation path. Common heuristic formulations that employ free space scattering assumptions for attenuation from fish groups are not in agreement with measurements here, and waveguide scattering theory is found to be necessary for accurate predictions. It is experimentally and theoretically shown that attenuation can be significant when the sensing frequency is near the resonance frequency of the shoaling fish, where scattering losses from the fish swimbladders and damping from fish flesh is most significant. Negligible attenuation was observed in previous OAWRS and POAWRS surveys because the frequency of the acoustic signals was sufficiently far from the swimbladder resonance peak of the shoaling fish or the packing densities of the fish shoals were not sufficiently high.
\end{abstract}

Thesis Supervisor: Nicholas Makris

Title: Professor of Mechanical and Ocean Engineering 
Massachusetts Institute of Technology 


\section{Acknowledgements}

I would like to express my appreciation to Prof. Nicholas Makris for the guidance and mentorship, and to my committee members Prof. Purnima Ratilal, Prof. George Barbastathis, and Dr. Daniel Zitterbart for their feedback on my research.

I would also like to thank the other members of the Laboratory for Undersea Remote Sensing for being so generous with their time, including Byung Gu Cho, Dong Hoon Yi, Wenjun Zhang, Shourav Pednekar, Arun Krishnadas, and Chenyang Zhu. Thank you to Geoffrey Fox for providing administrative support.

Thank you to all my friends I met at MIT, including Affi, Bitzy, Chika, Drew, Ian, Indy,

Jackie, Jenny, Margaret, PJ, Quantum, Rebecca, Sarah, Walker, and Xiaoyu. Thank you also to Tim and Deborah.

Most of all, thank you to mom, dad, and my brother Solomon for the nonstop love and support. 


\section{Funding Sources}

This work was supported by:

- Office of Naval Research under grant number N00014-17-1-2197.

- Office of Naval Research via the Graduate Traineeship Award under grant number N00014-18-1-2085. 


\section{Contents}

1 Introduction $\quad 35$

2 The Effect of Attenuation from Fish Shoals on Long-Range, Wide-Area Acoustic Sensing in the Ocean $\quad 39$

2.1 Introduction . . . . . . . . . . . . . . . . . . . . . . 39

2.2 Materials and Methods . . . . . . . . . . . . . . . . . . . 41

2.3 Results and Discussion . . . . . . . . . . . . . . . . . . . . . 48

2.3.1 Consistency between Backscattered Returns and Attenuation . . . . . 48

2.3.2 Attenuation Prediction and Frequency Selection . . . . . . . . . . . . 52

2.3.3 Estimating Scattering Strength with Attenuation . . . . . . . . . 55

2.4 Discussion . . . . . . . . . . . . . . . . . . . . . 56

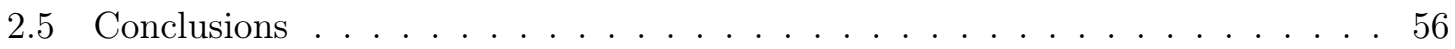

3 Quantification of Wide-Area Norwegian Spring-Spawning Herring Population Density with Ocean Acoustic Waveguide Remote Sensing $\quad 59$

3.1 Introduction . . . . . . . . . . . . . . . . . . . . . 59

3.2 Wide-Area OAWRS population density measurements without significant attenuation from herring ........................ 61

3.3 Correcting for attenuation from herring in wide-area OAWRS population density maps . . . . . . . . . . . . . . . . . . . . . . . . 62

3.4 Predicting sensing range reductions due to attenuation from fish . . . . . . . . 68

3.5 Spatial undersampling in echosounder surveys . . . . . . . . . . . . . . 70

3.6 Discussion . . . . . . . . . . . . . . . . . . . . . 71

3.7 Conclusions . . . . . . . . . . . . . . . . . . . . . . 75 
4 The Effect of Attenuation from Fish on Passive Ocean Acoustic Waveguide $\begin{array}{ll}\text { Remote Sensing of Surface Vessels in the Ocean } & 77\end{array}$

4.1 Introduction . . . . . . . . . . . . . . . . . . 77

4.2 Materials and Methods . . . . . . . . . . . . . . . . . . 79

4.2.1 Measuring Reductions in Ship Tonal Intensity due to Fish-Attenuation 79

4.2.2 Predicting Reductions in Ship Tonal Intensity due to Fish-Attenuation 81

4.3 Results. . . . . . . . . . . . . . . . . . . . . . 82

4.4 Discussion . . . . . . . . . . . . . . . . . . . . . . . . . 89

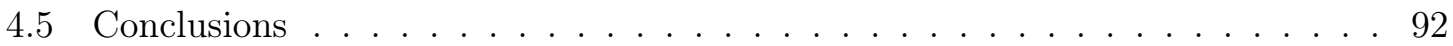

A Measurement of Scattering Strength in OAWRS Images $\quad 97$

B Evaluating whether Attenuation Effects are Present 101

C Modeling the Scatter Function of Individual Fish 103

D Modeling Two-Way Attenuation in a Waveguide Environment 105

$\begin{array}{ll}\text { E Derivation of Log-Likelihood Function } & 107\end{array}$

$\begin{array}{lr}\text { F Sound Speed Profiles } & 109\end{array}$

G Calibration of Target Strength $\quad 111$

H Measuring Sensing Range 115

I Correcting OAWRS transmissions for attenuation from fish 119

J The Effect of Attenuation from Fish on Ambient Noise 121

K Predicting Sensing Range in the Presence of Attenuation from Fish 125

L Synoptic Echosounder Measurements of Herring Areal Density and Depth $\begin{array}{ll}\text { Distribution } & 127\end{array}$

L.1 Measurements of herring population from OAWRS population density maps . 131

$\begin{array}{ll}\text { M Measured wind speed variations } & 135\end{array}$ 


\section{List of Figures}

2-1 Attenuation from multiple forward scattering is observable in Ocean Acoustic Waveguide Remote Sensing (OAWRS) scattering strength images of herring in Ålesund waters. Variation in bathymetry near Ålesund is shown in (A). The yellow dashed circle shows $60-\mathrm{km}$ diameter OAWRS areal coverage in 40 s. The red rectangular box represents the area investigated here. OAWRS scattering strength images assuming no scattering losses are shown from 21 February 2014 at 04:50:49 (B) and 04:33:19 (C). When the propagation path from the monostatic sensing system (black dot) to the distant shoal (Easting: 5-6 km, Northing: 0-4 km) has no occluding shoal, no attenuation is observable, as shown in (B). When an occluding shoal (Easting: 2-3 km, Northing: 2-4 km) is in the propagation path, the scattered returns from the same distant shoal are attenuated, as shown in (C) . . . . . . . . . . . . 46 
2-2 When an occluding fish shoal is uniformly distributed through the water column, attenuation in a waveguide has a form similar to that in free space in that the effect of attenuation appears as a single exponential factor in received intensity, as in Equation (2.14). In the example shown here, attenuation of each propagating mode $(\mathrm{A}-\mathrm{C})$ differs from free space attenuation by less than $0.5 \mathrm{~dB} / \mathrm{km}(\mathrm{D})$ and the decrease in sound pressure level due to attenuation $(\triangle S P L)$ matches the free-space-like factored approximation within $0.5 \mathrm{~dB}$ at any receiver depth (E). Here, attenuation during one-way propagation is modeled for Ålesund herring with expected scatter function $\langle S\rangle=-0.03+0.10 i$ in a shoal uniformly distributed between the sea surface and the seafloor (0-120 $\mathrm{m}$ ) with width $2 \mathrm{~km}$ and areal population density $2.5 \mathrm{fish} / \mathrm{m}^{2}$ estimated from echosounding data, with a source at depth $60 \mathrm{~m}$ with frequency $955 \mathrm{~Hz}$ and Ålesund sound speed profiles shown in Appendix F. Water density is modeled as $1000 \mathrm{~kg} / \mathrm{m}^{2}$, seafloor density as $1900 \mathrm{~kg} / \mathrm{m}^{2}$ and seafloor sound speed as

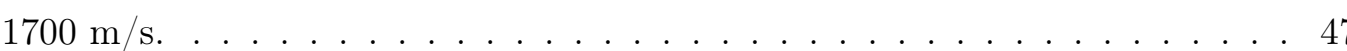


2-3 When an occluding fish shoal is concentrated at a specific water depth, attenuation will be complicated by waveguide effects so that the free-space-like factored approximation of Equation (2.14) may not be valid. Here, attenuation during one-way propagation is modeled for Ålesund herring shoals uniformly distributed between depths 0-40 m (red), 40-80 m (green) and 80$120 \mathrm{~m}$ (blue). Attenuation coefficients associated with given modes and their coupling depends on the specific modal contributions at the depths where the fish group is present $(\mathrm{A}-\mathrm{C})$. Since the environment modeled here is upward refracting, the lowest order modes are most attenuated when the herring occupy the upper water column (D) but they are essentially unattenuated when the herring occupy the lower water column $(\mathrm{H})$. In the case where herring are concentrated near the seafloor, a receiver in the upper water column will receive a strong signal from unattenuated lower order modes, causing the free-space-like factored approximation to overestimate the total attenuation $(\triangle S P L)$ by more than $5 \mathrm{~dB}(\mathrm{I})$. If the herring are concentrated near the sea surface (D-E) or near the center of the water column (F-G), the free-space-like factored approximation is seen to be in error by on the order of $2 \mathrm{~dB}$. Herring are modeled here with expected scatter function $\langle S\rangle=-0.03+0.10 i$ in a shoal with width $2 \mathrm{~km}$ and areal population density $2.5 \mathrm{fish} / \mathrm{m}^{2}$ estimated from echosounding data, with seafloor depth at $120 \mathrm{~m}$, source depth at $60 \mathrm{~m}$ with frequency $955 \mathrm{~Hz}$ and Ålesund sound speed profiles shown in Appendix F. Water density is modeled as $1000 \mathrm{~kg} / \mathrm{m}^{2}$, seafloor density as $1900 \mathrm{~kg} / \mathrm{m}^{2}$ and seafloor sound speed as $1700 \mathrm{~m} / \mathrm{s}$. . . . . . . . . . . . . . . 48 
2-4 Theoretical consistency is demonstrated between concurrently measured backscattering strength (A) and attenuation due to multiple forward scattering (B) from the occluding shoal shown in Figure 2-1. Attenuation is measured as the decrease in scattered returns from a distant shoal after the occluding shoal moves into the propagation path, that is the difference between the red and blue data in $(\mathrm{C})$. Modeled scattering strength and total attenuation (grey lines in A and B, respectively) assume the same fish shoal parameters and fit the data within one standard deviation. Scattering strength and attenuation of the occluding shoal (A and B) are modeled using the following estimated parameters: mean shoal depth $z_{0}=60 \mathrm{~m}$, shoal thickness $H=40 \mathrm{~m}$, neutral buoyancy depth $z_{n b}=0 \mathrm{~m}$, areal number density $n_{A}=2.4 \mathrm{fish} / \mathrm{m}^{2}$ and horizontal extent $r_{h}=1.1 \mathrm{~km}$. Scattering strength of the distant shoal (blue line in $\mathrm{C}$ ) is modeled using the following estimated parameters: mean shoal depth $z_{0}=85 \mathrm{~m}$, shoal thickness $H=50 \mathrm{~m}$, neutral buoyancy depth $z_{n b}=$ $10 \mathrm{~m}$ and areal number density $n_{A}=1.1 \mathrm{fish} / \mathrm{m}^{2} \ldots \ldots \ldots . \ldots 52$

2-5 The most favorable acoustic frequency for sensing in an ocean environment can be determined by maximizing the scattering strength uncorrected for two-way attenuation from fish $\left(S S-\triangle S P L_{2 w a y}\right)$ of a target. Here, $(S S-$ $\left.\triangle S P L_{2 w a y}\right)$ is modeled for fish shoals in four continental shelf environments: herring in the Gulf of Maine, herring in Ålesund waters, cod in Lofoten waters and capelin in Finnmark waters. The magnitude of attenuation depends on the width of the shoals along the propagation path between the sensing system and the target. When the shoal width along the propagation path is sufficiently small, attenuation will be negligible and the most favorable sensing frequency will be near swimbladder resonance. As the shoal width along the propagation path increases, there will be a tradeoff between frequencies near resonance where scattering strength is highest and off-resonance frequencies where attenuation is lowest. Red dotted lines designate the sensing frequency range of OAWRS experiments in each region. Physical parameters used for modeling each environment are shown in Table 2.1. . . . . . . . . . . . . . . 54 
2-6 The waveguide attenuation model can be used to account for scattering losses in acoustic backscattering data. Here, scattering strength images of herring shoals are shown from 04:33:19 on 21 February 2014 with (A) no scattering losses included and (B) scattering losses included. After accounting for scattering losses, the scattering strength of both the occluding shoal (Easting: 2-3 km, Northing: 2-4 km) and the distant shoal (Easting: $5-6 \mathrm{~km}$, Northing: 0-4 km) are comparatively uniform in space and are not biased by the horizontal extent of fish in the propagation path. Scattering losses are calculated using the following parameters: mean shoal depth $z_{0}=60 \mathrm{~m}$, shoal height $H=40 \mathrm{~m}$ and neutral buoyancy depth $z_{n b}=0 \mathrm{~m} \ldots \ldots \ldots$. . . . . 57

\section{3-1 Wide area OAWRS scattering strength map approximately one} hour before nautical sunset (2014 February 20, 17:55:49). Scattering strength is measured by correcting OAWRS transmissions for source level, areal resolution footprint, spreading loss and seafloor attenuation. The sensing range (thick white line) is approximately $20 \mathrm{~km}$. Denser fish groups appear at scattering strength levels above the labeled "Fish Shoaling Threshold" corresponding to the critical population density where herring groups were found to form $\left(0.2 \mathrm{fish} / \mathrm{m}^{2}\right)[37,38]$. Below the labeled "Seafloor Scattering Threshold" (0.05 fish $\left./ \mathrm{m}^{2}\right)$ fish groups are not reliably distinguishable from seafloor scattering. Regions between the seafloor scattering threshold and the fish shoaling threshold may include contributions from both dispersed fish groups and seafloor scattering. Regions beyond the sensing region (thick white line) are mostly dominated by ambient noise where $S P L-S L-T L A$ will increase with range, since transmission loss corrections are being applied to constant ambient noise. During this time there are few herring groups observed in echosounder measurements from the research vessel towing the OAWRS system (B), consistent with the OAWRS imagery in (A). The solid cyan line in (A) shows the path of the research vessel corresponding to the echogram shown, and the white dot shows the position of the monostatic OAWRS system. The cyan dotted line in (B) corresponds to the time when this OAWRS transmission was recorded. Thin white lines designate bathymetric contours. . 62 
3-2 OAWRS population density map of Norwegian spring spawning herring approximately one hour before nautical sunset (2014 February

20, 17:55:49). Herring shoals with population density above the $0.2 \mathrm{fish} / \mathrm{m}^{2}$ critical density where shoals were found to form [37, 38], are relatively sparsely distributed before sunset and have densities consistent with echosounder measurements during this time (Figure L-1). The average population density measured here is $0.07 \mathrm{fish} / \mathrm{m}^{2}$, where regions below the minimum detectable herring density $0.05 \mathrm{fish} / \mathrm{m}^{2}$ are not included in the measurement. Black lines designate bathymetric contours. . . . . . . . . . . . . . . . 64

3-3 Two hours after nautical sunset, several large, dense herring shoals are observed within the OAWRS sensing region, as well as a significant range-dependent decay caused by acoustic attenuation from the herring groups. An OAWRS transmission corrected for source level, areal resolution footprint, spreading loss and seafloor attenuation from 2014 February 20, 21:13:19 is shown in (A), where a significant range-dependent decay is observed. Many larger herring groups are seen to have formed after sunset in the OAWRS imagery, each often spanning a few kilometers, as confirmed by echosounder measurements from the research vessel towing the OAWRS system (B). The position of the OAWRS system is nearly identical to Figure 3-1, however the sensing range (thick white line) is reduced by $20 \%$ due to attenuation from fish. While herring groups can be visually observed in the scattering strength map shown, it is difficult to determine which regions are dominated by scattering from fish or seafloor without correcting the OAWRS imagery for attenuation from fish. The solid cyan line in (A) shows the path of the research vessel corresponding to the echogram shown, and white dot shows the position of the monostatic OAWRS system. The cyan dotted line in (B) corresponds to the time when this OAWRS transmission was recorded. Thin white lines designate bathymetric contours. . . . . . . . . 64 


\section{3-4 The range-dependent decay in OAWRS transmissions after nau-}

tical sunset is found to be consistent with theoretical predictions for attenuation from fish, which can be used to correct OAWRS images for fish attenuation. The theoretical decay due to fish depends on the average population density within the sensing region, which is determined for each OAWRS transmission by modeling scattering strength uncorrected for losses from fish attenuation ("fish-attenuated scattering strength") and performing a least-squares fit with measurements (A-C). Fish-attenuated scattering strength is measured for each OAWRS transmission by correcting for source level, areal resolution footprint, spreading loss and seafloor attenuation and averaging across azimuthal angle (Appendix I). Before nautical sunset, measurements of fish-attenuated scattering strength do not significantly change with range (black line in A). After nautical sunset, an increase in fish-attenuated scattering strength is observed at ranges below $6 \mathrm{~km}$, as well as a significant decay with range (black lines in B-C). Fish-attenuated scattering strength is modeled assuming a horizontally uniform distribution of herring with species-specific parameters shown in Table 3.1. The average areal number density of herring within the sensing range of the OAWRS system is determined by performing a least-squares fit between measured and modeled fish-attenuated scattering strength (red lines in A-C). The log-likelihood function is calculated by integrating the weighted difference squared across range in polar coordinates (Equation (A3), where measurements at longer ranges are weighted more heavily since they correspond to a larger area. Average population density significantly increases in the hours after nautical sunset, from approximately $0.08 \mathrm{fish} / \mathrm{m}^{2}$ before $18: 30$ to nearly $0.3 \mathrm{fish} / \mathrm{m}^{2}$ after 21:00

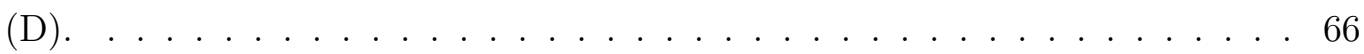




\section{3-5 OAWRS population density map of Norwegian spring spawning her-}

ring approximately two hours after nautical sunset (2014 February

20, 21:13:19). Here a correction has been applied to account for attenuation from fish, where the modeled decay is estimated from the average areal density within the sensing region calculated in Figure 3-4. Multiple large herring groups are observed with dimensions of several kilometers and population densities of up to roughly $5 \mathrm{fish} / \mathrm{m}^{2}$, which is consistent with echosounder measurements during this time (Figure L-2). The average population density measured here is 0.29 fish $/ \mathrm{m}^{2}$, where regions below the minimum detectable herring density $0.05 \mathrm{fish} / \mathrm{m}^{2}$ are not included in the measurement. The color scale is chosen so that the transition from brown to blue occurs at $0.2 \mathrm{fish} / \mathrm{m}^{2}$, which is the critical population density at which large herring shoals were found to form $[37,38]$. Black lines designate bathymetric contours. . . . . . . 68

3-6 The increase in herring population density at nautical sunset is correlated with a reduction in sensing range for the OAWRS system. Before 18:00, the average areal population density of herring (blue data) is approximately $0.07 \mathrm{fish} / \mathrm{m}^{2}$, and the sensing range (red data) remains stable at approximately $20 \mathrm{~km}$. Over the course of the two hours following nautical sunset, the average areal density of herring within the sensing region increases to nearly $0.3 \mathrm{fish} / \mathrm{m}^{2}$, corresponding with a $20 \%$ reduction in sensing range. . 69 


\section{3-7 Reductions in ambient noise level observed after nautical sunset}

are consistent with predicted reductions in ambient noise due to attenuation from fish. The decrease in ambient noise $(\Delta N L$, black data in B) is measured as the difference between the mean ambient noise level before nautical sunset $N L_{1}$ (17:00-18:30, blue data in A) and after nautical sunset $N L_{2}$ (19:30-21:00, magenta data in A). Ambient noise measurements before and after nautical sunset are separated by more than a standard deviation $\left(N L_{1}+\sigma_{1}<N L_{2}-\sigma_{2}\right)$ for frequencies within in an $890 \mathrm{~Hz}$ band near the swimbladder resonance peak of the herring (black dotted lines in A). The solid black line in (B) denotes measured $\Delta N L$ for frequencies within this band, and the dotted black lines in (B) denote measured $\Delta N L$ for frequencies outside of this band. The modeled reduction in ambient noise from fish depends on the average areal population density before nautical sunset $\left(n_{A 1}\right)$ and after sunset $\left(n_{A 2}\right)$, which are respectively determined from the mean of OAWRS measurements of average population density from 17:00-18:30 and 19:30-21:00 (Figure 3-4D). Solid lines in (A) show the mean ambient noise level within each time frame, and shaded patches denote the standard deviation. The dominant source of ambient noise at the frequencies studied is surface agitation from wind $[58] . \ldots \ldots \ldots$. . . . . . . . . . 70 
3-8 Sensing range predictions are shown for multiple environments where fish groups are known to shoal, and theoretical predictions are found to be in agreement with sensing range measurements for the OAWRS system. Expected sensing range is shown in the presence of $(A)$ herring shoals in Ålesund waters, (B) capelin shoals in Finnmark waters, and (C) cod shoals in Lofoten waters. Sensing range predictions depend on the input source power of the sensing system and the ambient noise level. Sensing range is modeled in each environment for $W_{0}-N L_{0} \pm 10 \mathrm{~dB}$ re $1 \mu \mathrm{Pa}$, where $W_{0}$ is the experimental input source power used and $N L_{0}$ is the experimental noise level. Measured sensing range for the OAWRS system in the presence of Ålesund herring and Finnmark capelin (red dots in A and B) are shown to be in agreement with expected sensing range at the relevant $W_{0}-N L_{0}$ values (red lines in A and B). Physical parameters used for modeling sensing range for Finnmark capelin and Lofoten cod are shown in Table 1 of [15]. Sensing range predictions for Lofoten cod exceed the maximum possible range that could be recorded in the 50 second recording window that was used in this region (red dot in $\mathrm{C}$ ). Here the received signal is assumed to be dominated by scattering from fish. . . . . . . . . . . . . . . . 7

3-9 Sample instantaneous OAWRS images of herring shoals near Ålesund trench, a historic spawning ground, over a seven hour period (2014 February 21 (01:02:39-08:33:19). On February 21 before nautical sunrise (6:30), a large, elongated herring shoal is observed on the northeastern edge of the trench with a total population on the order of 4 million (A-B). Five minutes after nautical sunrise, this large shoal begins to fragment $(\mathrm{C})$. Two hours after nautical sunrise, the shoal has dissipated (D). The measured population size within the survey box for each OAWRS image shown here is in Figure 3-10. The color scale is chosen so that the transition from brown to blue occurs at $0.2 \mathrm{fish} / \mathrm{m}^{2}$, which is the critical population density at which large herring shoals were found to form $[37,38] \ldots \ldots$. . . . . . . . . . . 72 
3-10 Herring population from instantaneous OAWRS imagery over entire survey region shown in Figure 3-9 for each instant as a function of time. Herring population is calculated by integrating OAWRS measurements of population density over regions where the population density is greater than $0.2 \mathrm{fish} / \mathrm{m}^{2}$, which is the critical population density at which large herring shoals were found to form $[37,38]$ (Appendix L.1) . . . . . . . . . . . . . . 73

3-11 Spatial herring distribution inhomogenieties seen in wide-area OAWRS imagery are undersampled in sparse line-transect surveys and lead to population estimates ranging from 0.5 to 2.5 times the total OAWRS population for the region shown. Assuming the spatial distribution of herring found in OAWRS population density maps and echosounder tracks from a 2001 survey in the same region (black line in A) [48], echosounder estimates of herring population are simulated by multiplying the average areal density along the echosounder transect within the survey region with the area of the survey region. The yellow contour in (A) denotes the OAWRS survey region for February 20, 17:55:49 (Figure 3-2), the blue contour in (A) denotes the OAWRS survey region for 21:13:19 (Figure 3-5), and the green box in (A) denotes the data collection site for February 21, 1:00-8:35 (Figure 3-9). Simulated echosounder population estimates as a percentage of OAWRS measurements are shown in (B), where yellow, blue, and green data correspond to simulations of echosounder estimates within the corresponding OAWRS

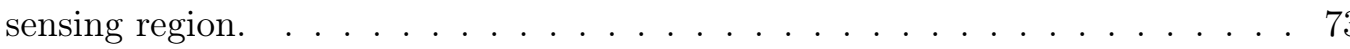

4-1 Ship-radiated tonals from the FV Artus (red) are intermittently detected by a horizontal coherent receiver array towed by the RV Knorr (orange) in herring spawning grounds near Ålesund, Norway between 3:10 and 6:00 UTC on February 21, 2014. Red and orange dots denote the starting position of the tracks at $3: 10$ UTC . . . . . . . . 83 


\section{4-2 Significant reductions in the intensity of a ship-radiated tonal from}

the FV Artus are observed when a group of three herring shoals occlude the propagation path. At 3:14:59, no fish groups are observed in wide-area OAWRS imagery along the propagation path from the Artus to the receiver array (A). At 3:26:39, OAWRS imagery reveals three fish shoals occluding the propagation path (B). Spectrograms of the $1584 \mathrm{~Hz}$ tonal from the Artus beamformed in the direction of the Artus are shown in (C) and (D), where significant reductions in the received level are observed after the fish groups occlude the propagation path. The received level within the bandwidth of the Artus tonal $(R L)$ corrected for transmission loss from spreading and seafloor attenuation $(T L)$ is shown in $(\mathrm{E})$ and $(\mathrm{F})$. Blue dots denote $R L+T L$ measurements averaged over $1 \mathrm{~s}$ intervals within the frequency band of the Artus tonal (1569-1599 Hz), and black lines denote the mean and standard deviation of these $1 \mathrm{~s}$ measurements. The three white lines in (B) correspond to echosounder transects of the three herring shoals shown in Figure 4-3. Fluctuations in the measured scattering strength of herring shoals between (A) and (B) are caused by attenuation from fish to OAWRS signals, which has been previously investigated in [15]. Reductions in the received level between $1500-1700 \mathrm{~Hz}$ (C and D) are likely caused by attenuation from herring of broadband noise from the Artus. Black dotted lines in (A) and (B) designate bathymetric contours. . . . . . . . . . . . . . . 84 


\section{4-3 In order to predict reductions in ship-radiated tonals from the FV}

Artus due to attenuation from herring, the areal population density, vertical position, and horizontal position of the three herring shoals labeled in Figure 4-2B are characterized using a combination of in situ echosounder measurements and OAWRS imagery. The areal population density and vertical distribution are estimated from echosounder measurements of the shoals recorded less than two hours from the OAWRS transmission (A-C). The areal density along each echosounder transect is shown in (D-F). The three shoals are segmented by choosing regions where the measured areal population density is above the critical population density at which herring groups were found to form $\left(0.2 \mathrm{fish} / \mathrm{m}^{2}\right.$, black solid lines in D-F) $[37,38]$. The vertical position of these shoals is determined by selecting depths where the average volumetric population density along each segment is greater than $10^{-3} \mathrm{fish} / \mathrm{m}^{2}$ (black solid lines in G-I). The horizontal position of the shoals along the propagation path from the FV Artus to the receiver is estimated from OAWRS measurements of fish-attenuated scattering strength along the propagation path $(\mathrm{J})$. The horizontal position of the shoals (gray boxes in $\mathrm{J}$ ) is determined by selecting regions where the fish-attenuated scattering strength is $5.6 \mathrm{~dB}$ greater than the mean seafloor

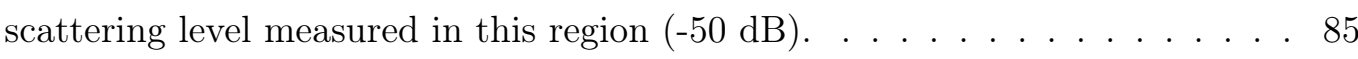




\section{4-4 The predictions of the waveguide attenuation model are consistent}

with measured reductions in the Artus tonal due to attenuation from herring. The population density distributions of the three shoals measured in Figure 4-3 as well as the position of the source and receiver are shown in (A). A source depth of $6 \mathrm{~m}$ (magenta dot in $\mathrm{A}$ ) is chosen to correspond with the draft depth of the FV Artus [22], and the depth of the receiver array towed by the RV Knorr is $50 \mathrm{~m}$ (white dot in A). Attenuation coefficients associated with given modes and their coupling depend on the specific modal contributions at the depths where the fish groups are present (B-D). Since the Ålesund underwater environment is upward-refracting (Figure F-1), the lowest order modes concentrated in the upper water column will experience almost no attenuation from the fish groups which are concentrated in the bottom half of the water column (E). As a result, the predicted reduction in sound pressure level due to attenuation from fish $(\triangle S P L)$ is approximately $10 \mathrm{~dB}$ lower when the receiver is in the upper water column compared to the lower water column (F). The modeled decrease in the received level (blue solid line in F) is within a standard deviation of measurements at the relevant receiver depth (red data in $\mathrm{F}$ ). The loss due to attenuation estimated by the free-space-like factored approximation is not sensitive to changes in receiver depth, and predictions are more than $5 \mathrm{~dB}$ greater than measurements (blue dotted line in F). Water density is modeled as $1000 \mathrm{~kg} / \mathrm{m}^{2}$, seafloor density as $1900 \mathrm{~kg} / \mathrm{m}^{2}$ and seafloor sound speed as $1700 \mathrm{~m} / \mathrm{s} \ldots \ldots$. . . . . . . . . 87 
4-5 OAWRS map of herring areal population density between 3:00 and 6:00. Since attenuation from fish prevented the entire region from between instantaneously surveyed, the areal population density map is generated from five instantaneous OAWRS transmissions between 3:00 and 6:00 on February 21, 2014 (3:04:09, 3:24:09, 4:19:09, 5:04:09, and 5:39:59) according to Appendix A. Reductions in the received level of the Artus tonal between 3:10 and 6:00 shown in Figure 4-7 are calculated using the population density between the Artus (pink line) and the Knorr (black line). The color scale is chosen so that the transition from brown to blue occurs at $0.2 \mathrm{fish} / \mathrm{m}^{2}$, which is the critical population density at which large herring shoals were found to form [37, 38]. Black dotted lines denote bathymetric contours. . . . . . . . . . . . . . . . 90

\section{4-6 Detections of ship-radiated tonals from the FV Artus are lost to} ambient noise when dense herring shoals occlude the propagation path. Spectrograms of the received level beamformed in the direction of the Artus are shown in (A) and (F). At approximately 3:28 on February 21, the signal from the Artus falls below ambient background noise (A). Before this loss of detection, OAWRS measurements of herring population density between the source and receiver are mostly below $0.2 \mathrm{fish} / \mathrm{m}^{2}$, which is the critical population density at which large herring shoals were found to form $(\mathrm{B}, \mathrm{C})[37,38]$. After the loss of detection, multiple fish groups are observed along the propagation path with population densities above $0.2 \mathrm{fish} / \mathrm{m}^{2}$ (red data in $\mathrm{D}, \mathrm{E})$. The signal from the Artus is detected again at approximately 5:10 (F). Before detection at 5:10 there are still dense fish groups occluding the propagation path with population densities above $0.2 \mathrm{fish} / \mathrm{m}^{2}$ (red data in $\mathrm{G}, \mathrm{H})$. After the Artus is detected, the population densities are below 0.2 fish $/ \mathrm{m}^{2}(\mathrm{I}, \mathrm{J})$. Measurements of herring areal population density shown here are from the OAWRS population density map shown in Figure 4-5. Vertical white lines in $(\mathrm{A})$ and $(\mathrm{F})$ correspond to times when measurements in (BE) and (G-J) are respectively made. Broadband noise from the Artus is also observed between 3:00 and 3:28 in (A), and the strong broadband signal between 5:17 and 5:35 in (F) is likely caused by propeller cavitation from the

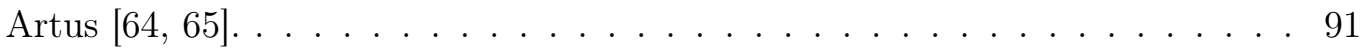




\section{4-7 Reductions in the received level of the Artus tonal between 3:10 and}

6:00 on February 21 and corresponding detection losses are consistent with predicted reductions due to attenuation from fish. The received level beamformed in the direction of the Artus within the frequency band of the Artus tonal $(1569-1599 \mathrm{~Hz})$ is shown in (A). Red data indicates times when the Artus tonal was detected above background noise, while black data indicates times then the Artus tonal was not detected (Appendix N). The received level corrected for transmission loss from spreading losses and seafloor attenuation $(R L+T L)$ is shown in (B) for data where the Artus tonal is detected above ambient noise. The reduction in received level due attenuation from fish $(\triangle S P L)$ is predicted in (C) using the OAWRS map of herring population density shown in Figure 4-5. The measured $4 \mathrm{~dB}$ reduction in $R L+T L$ between 3:15 and 3:26 (B) is consistent with the predicted loss due attenuation from fish during this time (C). After the detection loss at 3:28, the signal remains undetected until the predicted loss due to attenuation $(\triangle S P L)$ falls below $2 \mathrm{~dB}$ after 5:00. The gap in the data between 3:38 and 4:15 is from a period where both the RV Knorr and the FV Artus are turning. The gap in the data between 5:15 and 5:20 is from a period where the Artus is turning. The approximately $4 \mathrm{~dB}$ increase in $R L+T L$ after this gap in (B) is likely caused by this change in orientation, since the Artus turns away from the Knorr and creates a more direct path from the Artus propellor

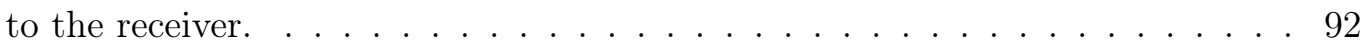


4-8 The decrease in the intensity of ship-radiated tonals due to attenuation from fish $(\triangle S P L)$ is predicted here for previous POAWRS surveys in three continental shelf environments: herring in the Gulf of Maine, capelin in Finnmark waters, and herring in Ålesund waters. The magnitude of $\triangle S P L$ depends on the width of the shoals along the propagation path between the source and receiver. Tonals from the RV Delaware II (black dotted lines in A) are well below the resonance peak of the herring in the Gulf of Maine and predicted losses due to attenuation are less than $0.3 \mathrm{~dB}$. The width of the observed capelin shoals in Finnmark are less than $0.2 \mathrm{~km}$, and corresponding losses due to attenuation are predicted to be less than $1 \mathrm{~dB}$ at the frequencies of tonals from the RV Johan Hjort (black dotted lines in B). Predicted attenuation from herring in Ålesund is significant at the frequency of the tonal from the FV Artus (black dotted line in C). Physical parameters used for modeling attenuation in each environment are shown in Table 2.1, and measured sound speed profiles in each environment are shown in Figure F-1. . . . . . . . . . . . . . . . . . . . 94

A-1 OAWRS system used for herring measurements during the Nordic Seas 2014 Experiment [35]. The system is effectively monostatic with source and receiver arrays towed from the same research vessel (RV Knorr). The OAWRS source was developed under the National Science Foundation and Sloan Foundation MRI program for wide-area sensing of marine life, and the ONR Five Octave Research Array (FORA) was used as the OAWRS receiver. . . . . . . . . . . 99 
B-1 Example OAWRS scattering strength images of fish shoals assuming no scattering losses are shown from four seperate continental shelf environments (A, C, E, and G). Scattering strength levels along radial transects that bisect fish shoals are shown in $\mathrm{B}, \mathrm{D}, \mathrm{F}$, and $\mathrm{H}$. The positions of the radial transects are given by white lines in A, C, E, and G. We see evidence of attenuation through Ålesund herring shoals (blue line in B) since there is a sharp increase in scattering strength where the shoal begins $(2.5 \mathrm{~km}$ from the source/receiver) followed by a steady decease in scattering strength caused by attenuation as range increases. After applying the attenuation correction described in Section 2.3.3 this effect is no longer present (red line in B). We do not see evidence of attenuation from Gulf of Maine herring, Finnmark capelin, and Lofoten cod (D, F, and $\mathrm{H}$, respectively) since there is no steady decrease in scattering strength as range increases, and no attenuation correction is necessary. Sensing frequencies for the scattering strength images shown here are $955 \mathrm{~Hz}$ for Ålesund herring, $950 \mathrm{~Hz}$ for Gulf of Maine herring, $955 \mathrm{~Hz}$ for Lofoten cod, and $1335 \mathrm{~Hz}$ for Finnmark capelin. Black dotted lines indicate water depth contours. . . . . . . . . . . . . . . . . . . . 102

F-1 Profiles of water-column sound speed from XBT measurements in the Gulf of Maine (A), Ålesund waters (B), Lofoten waters (C), and Finnmark waters (D).110 
G-1 The target strength of herring in Ålesund is determined by calibrating OAWRS scattering strength measurements of a large, elongated herring shoal observed on February 21 with nearly concurrent echosounder transects. The OAWRS scattering strength map from February 21, 2014 at 3:26:39 at sensing frequency $955 \mathrm{~Hz}$ is shown in (A) with the echosounder transect from 2:52:002:58:00 overlain in white. Echosounder data is shown in (B), where vertical black dotted lines denote the transect studied here. The average areal population density along the echosounder transect is shown in (C), and average volume density of herring with respect to depth along this transect is shown in (D). The target strength of herring in this shoal is modeled assuming the depth distribution shown in (D), and neutral buoyancy depth is determined to be $3 \mathrm{~m}$ by performing a least-squares fit between measured and modeled target strength $(\mathrm{E}) \ldots \ldots \ldots \ldots$. . . . . . . . . . . . . . . . . . . . . . . . .

\section{H-1 Reductions in signal intensity and ambient noise caused by atten-} uation from herring groups resulted in a $20 \%$ reduction in sensing range after nautical sunset. "Sensing range" is defined here as the range at which scattered returns from the environment can be observed above ambient noise. OAWRS maps of normalized pressure level are generated by averaging 12 instantaneous OAWRS images at $955 \mathrm{~Hz}$ at 17:00-17:10 (A) and 21:00-21:00 (B). The mean sensing range over each hour-long interval is calculated by averaging each sound pressure level map across all azimuthal angles, excluding angles within $25^{\circ}$ of endfire (C-D). Ambient noise (black dotted lines in $\mathrm{C}-\mathrm{D}$ ) is measured for each time interval as the average sound pressure level above $29.5 \mathrm{~km}$, where sound pressure level is flat enough to be considered dominated by ambient noise (Equation A4). Sensing range (red dots in C-D) is measured as the range where sound pressure level (blue lines in C-D) falls within the detection threshold $D T=5.6 \mathrm{~dB}$ of the ambient noise. Over the course of the four hours shown here, sensing range reduces by 20\%. Light blue patches in C-D denote the standard deviation of normalized pressure level. The pressure levels shown here are normalized by the ambient noise level measured between 17:00 and 17:10 (A and B) . . . . . . . . . 117 
L-1 Synoptic echosounder measurements of Ålesund herring shoals are consistent with OAWRS population density measurements before nautical sunset. In (A), bathymetric contours are shown in black, the path of the research vessel towing the OAWRS system (RV Knorr) between 17:00 and 18:30 on February 20, 2014 is overlain in blue, and the path of the research vessel from which echosounder measurements were recorded during this time is overlain in magenta. Echosounder measurements of volumetric population density are shown in (B), and measurements of areal population density are shown in (C). Sparse herring groups are observed with population densities on the order of $0.3 \mathrm{fish} / \mathrm{m}^{2}$, which is consistent with OAWRS population density measurements during this time (Figure 3-2) . . . . . . . . . . . . . . . 128

L-2 Synoptic echosounder measurements of Ålesund herring shoals are consistent with OAWRS population density measurements after nautical sunset. In (A), bathymetric contours are shown in black, the path of the research vessel towing the OAWRS system (RV Knorr) between 19:30-21:00 on February 20, 2014 is overlain in blue, and the path of the research vessel from which echosounder measurements were recorded during this time is overlain in magenta. Echosounder measurements of volumetric population density are shown in (B), and measurements of areal population density are shown in (C). Several dense herring groups are observed with population densities on the order of $5 \mathrm{fish} / \mathrm{m}^{2}$, which is consistent with OAWRS population density measurements during this time (Figure $3-5) \ldots \ldots$. . . . . . . . . . 129

L-3 Probability density function of herring depth $p(z)$ inverted from echosounder measurements on 2014 February 20 between 17:00 and 21:00 in Ålesund spawning grounds (Equation A4) . . . . . . . . . . . . . . . . 129

L-4 Probability density function of herring depth $p(z)$ inverted from echosounder measurements on 2014 February 21 between 3:10 and 6:00 in Ålesund spawning grounds $($ Equation A4) . . . . . . . . . . . . . . . . 130 
L-1 Echosounder measurements confirm that the majority of herring contained in the discrete shoals observed by OAWRS are in regions where the population density is greater than the critical population density $0.2 \mathrm{fish} / \mathrm{m}^{2}[37,38]$. An OAWRS population denisty map from February 21, 3:26:39 is shown in (A), where the path of an echosounder is overlain in white and the corresponding echosounder data from 2:48-3:40 are shown in (B). The green dot in (A) and the green line in (B) correspond to the echosounder position for the OAWRS image shown in (A). Black dotted lines in (B) designate regions $\alpha$, $\beta$, and $\gamma$, where both systems co-register dense fish groups (A). The areal population density measured by the echosounder along this transect is shown in $(\mathrm{C})$, where red data denotes regions where the OAWRS population density is greater than $0.2 \mathrm{fish} / \mathrm{m}^{2}$ and black data denotes regions where the OAWRS population density is less than $0.2 \mathrm{fish} / \mathrm{m}^{2}$. It is found that selecting regions above the $0.2 \mathrm{fish} / \mathrm{m}^{2}$ threshold is an effective way to segment discrete herring shoals (red data in C) without including measurements potentially affected by scintillation and contamination from background seafloor scattering (black data in $\mathrm{C}$ ). By contrast, a significant portion of OAWRS data above 0.05 fish $/ \mathrm{m}^{2}$ falls outside the dense, discrete shoal (D) . . . . . . . . . . . . . 131

L-2 The percentage of the herring population measured by echosounders between February 17, 23:30 and February 21, 21:00 above the population density threshold $n_{A}$ is shown here. $87 \%$ of the herring population is found to be above the critical population density $0.2 \mathrm{fish} / \mathrm{m}^{2}$ (red dotted line). . . . . . . 133

M-1 Wind speed measurements recorded by the research vessel towing the OAWRS system (RV Knorr) are shown here from February 20, 2014 between 17:0018:30 (before nautical sunset) and between 19:30-21:00 (after nautical sunset). There is no statistically significant difference between wind speed before and after sunset, indicating that reductions in ambient noise after sunset are not caused by changes in wind speed. . . . . . . . . . . . . . . . 135

O-1 The speed of the Artus between 3:10 and 6:00 on February 21, 2014 is shown here. With the exception of the ship turns at 4:09-4:25, and 5:18-5:33, variations in the speed of the Artus are smaller than $0.5 \mathrm{~m} / \mathrm{s} \ldots \ldots . . . . . .140$ 


\section{Chapter 1}

\section{Introduction}

Acoustics is the primary means of sensing targets in the ocean such as manmade vehicles, geophysical phenomena, and vocalizing marine mammals [40, 53, 55]. Attenuation from fish, however, can reduce the intensity of acoustic signals and significantly decrease detection range for active and passive sensing in the ocean. This makes it important to understand the relevant mechanisms and accurately predict attenuation from fish in underwater acoustic sensing. Here, Ocean Acoustic Waveguide Remote Sensing (OAWRS) is used to instantaneously image massive Norwegian herring shoals that stretch for thousands of square kilometers and experimentally measure reductions in active and passive signals due to attenuation from these shoals.

Most previous experimental measurements of attenuation from fish have relied on fluctuations in the intensity of long-range acoustic signals caused by diel or seasonal shoaling patterns of fish along the propagation path, including sardines in the Bristol Channel and groups of anchovies and sardines in the Gulf of Lion $[59,7,12,13]$. In these experiments fish population densites were inferred from the reductions in signal intensity, since it is difficult to measure instantaneous population distributions across long ranges with conventional survey methods. While some of these experiments included synoptic echosounder measurements of fish density and vertical distribution [12,13], they did not include direct observations of the fish groups occluding the propagation path. A later study predicted that attenuation from sardine shoals off the west coast of the United States would be significant using measurements of shoal size and population density, but the study did not include experimental measurements of acoustic attenuation to compare with predictions [32]. Here, OAWRS is 
used to instantaneously image fish shoals that stretch for thousands of square kilometers and simultaneously measure attenuation from these shoals within the active OAWRS transmissions, as well as attenuation to ship-radiated tonals detected by Passive Ocean Acoustic Waveguide Remote Sensing (POAWRS).

Previous OAWRS experiments have revealed the diel shoaling patterns of massive fish groups, including vertical migrations of shoaling herring measured from changes in the resonance frequency of the fish as they move from deep water to their spawning locations [61]. OAWRS has also revealed that shoaling fish tend to rapidly congregate in massive groups spanning tens of kilometers when the fish population density reaches a species-specific critical value [37]. Shoal growth was found to propagate horizontally outward in compressional waves at speeds orders of magnitude larger than the swimming speed of a fish, indicating that shoal formation is the result of synchronous convergence of individual fish [37, 38]. These massive fish groups are found to have a relatively stable mean size, and historical population surveys have shown that when a given species was overfished to the point where the total spawning population fell within a standard deviation of the group size, a return to pre-industrial total spawning populations took decades [35]. OAWRS has also been used to observe ecosystem-scale interactions between fish groups and marine mammals that forage for fish, and it was shown that marine mammals will spatially converge on fish spawning grounds and divide into separate foraging areas specific to each marine mammal species [55].

In Chapter 2 of this thesis, it is experimentally shown with wide-area OAWRS over $360^{\circ}$ in the horizontal and ranges spanning many tens of kilometers that a single large fish shoal can significantly occlude acoustic sensing over entire sectors spanning more than $30^{\circ}$ with corresponding decreases in signal intensity by roughly an order of magnitude. Such blockages can comprise significant impediments to underwater acoustic remote sensing and surveillance of underwater vehicles, marine life and geophysical phenomena as well as underwater communication. This makes it important to understand the relevant mechanisms and accurately predict attenuation from fish in long-range underwater acoustic sensing and communication. To do so, we apply an analytical theory derived from first principles for acoustic propagation and scattering through inhomogeneities in an ocean waveguide to model propagation through fish shoals. We find experimentally and theoretically that attenuation can be significant when the sensing frequency is near the resonance frequency of the shoaling fish. Negligible attenuation was observed in previous low-frequency ocean acoustic waveguide 
remote sensing (OAWRS) experiments because the sensing frequency was sufficiently far from the swimbladder resonance peak of the shoaling fish or the packing densities of the fish shoals were not sufficiently high. We show that common heuristic approaches that employ free space scattering assumptions for attenuation from fish groups can lead to significant errors for applications involving long-range waveguide propagation and scattering.

Norwegian spring-spawning herring is a critical economic resource for multiple nations in the North Atlantic, and a keystone species of the Nordic Seas ecosystem. Given the wide areas the herring occupy, it is difficult to accurately measure population size and spatial distribution. In Chapter 3, OAWRS is used to instantaneously measure the areal population density of Norwegian herring over more than one thousand square kilometers in spawning grounds near Ålesund, Norway. In the vicinity of the Ålesund trench near peak spawning, significant attenuation in signal-to-noise ratio and mean sensing range is observed after nautical sunset that has not been observed in previous OAWRS surveys of in the Nordic Seas or in other regions. We show this range-dependent decay along a given propagation path is caused by attenuation through dense herring shoals forming at sunset and persisting through the evening for transmissions near the swimbladder resonance peak. OAWRS transmissions are corrected for attenuation in a manner consistent with waveguide scattering theory and simultaneous downward directed local line-transect measurements in the region in order to produce instantaneous wide-area population density maps. Corresponding measured reductions in median sensing range over azimuth before ambient noise limitation are shown to be theoretically predictable from waveguide scattering theory and observed population densities. Spatial-temporal inhomogenieties in wide-area herring distributions seen synoptically in OAWRS imagery show that standard sparsely spaced line transect surveys through this region during spawning can lead to large errors in estimated population due to spatial and temporal undersampling.

In Chapter 4, the effect of attenuation from herring shoals on the Passive Ocean Acoustic Waveguide Remote Sensing (POAWRS) of surface vessels is investigated, where concurrent wide-area active OAWRS is used to confirm that herring shoals occluding the propagation path are responsible for measured reductions in ship radiated sound and corresponding detection losses. Reductions in signal intensity are predicted using the normal-mode-based analytical theory for acoustic propagation and scattering through inhomogeneities in an ocean waveguide. The predictions of the waveguide attenuation formulation are in agreement 
with measured reductions from attenuation, where the position, size, and population density of the fish groups are characterized using OAWRS imagery as well as in situ echosounder measurements of the specific shoals occluding the propagation path. Common heuristic formulations that employ free space scattering assumptions for attenuation from fish groups are not in agreement with measurements here. Waveguide scattering and propagation theory is found to be necessary for accurate predictions. 


\section{Chapter 2}

\section{The Effect of Attenuation from Fish Shoals on Long-Range, Wide-Area Acoustic Sensing in the Ocean}

\section{$2.1 \quad$ Introduction}

Acoustics is the primary means of long-range and wide-area sensing in the ocean due to the severe attenuation of electromagnetic waves in seawater. While it is known that densely packed fish groups can attenuate acoustic signals during long-range propagation in an ocean waveguide, previous experimental demonstrations have been restricted to single line transect measurements of either transmission or backscatter and have not directly investigated wide-area sensing and communication issues. Here we analyze the effects of attenuation from multiple forward scattering on instantaneous imaging of fish population densities over thousands of square kilometers with Ocean Acoustic Waveguide Remote Sensing (OAWRS) $[26,37,38]$. We experimentally show with wide-area sensing over $360^{\circ}$ in the horizontal and ranges spanning many tens of kilometers that a single large fish shoal can significantly occlude acoustic sensing over entire sectors spanning more than $30^{\circ}$ with corresponding decreases in detection ranges by roughly an order of magnitude. Such blockages can comprise significant impediments to underwater acoustic remote sensing and surveillance of underwater vehicles and geophysical phenomena as well as underwater communication. Understanding the effects of attenuation from fish is also important for conservation efforts. 
Climate change and overfishing have led to massive declines in marine populations, with an estimated 33 percent of marine fish stocks being harvested at unsustainable levels [52]. In order to effectively regulate fishing quotas and stabilize marine populations, it is increasingly important to develop methods for accurately surveying and monitoring fish populations over ecosystem scale areas. Low frequency $(<3 \mathrm{kHz})$ acoustic sensing systems currently provide the only means of instantaneously sensing fish populations over kilometer-scale areas [37, 38], however attenuation within fish shoals can reduce the strength of acoustic signals and bias population density estimates.

The potential limitations in detection range imposed by attenuation from fish makes it important to understand the relevant mechanisms and accurately predict attenuation from fish. To do so, we apply an analytical theory derived from first principles for acoustic propagation and scattering through inhomogeneities in an ocean waveguide [43] to model propagation through fish shoals. Our formulation treats multiple scatter in the forward direction. Multiple scattering in non-forward directions was found to be negligible given the packing densities and target strengths of the fish shoals we encountered based on the work of Andrews et al. [2].

The formulation used here for modeling attenuation combines waveguide scattering theory $[25,36]$ and a differential slab marching approach introduced by Rayleigh for the mean field to derive the first two statistical moments of the acoustic field in a waveguide with inhomogeneities [43]. This formulation has been previously shown to be consistent with experimental measurements of attenuation and temporal coherence loss in the presence of internal waves for both mid-frequency signals $(415 \mathrm{~Hz})$ in a continental shelf environment and low-frequency signals $(10-70 \mathrm{~Hz})$ in a deep ocean waveguide $[4,5,20]$.

In previous experiments, either the attenuation from fish in the shoal or the scattering cross sections of fish in the shoal were measured but not both, making it impossible to directly confirm a theoretical prediction on attenuation through the shoal [7, 42]. Here, both measurements have been made and they experimentally confirm the waveguide theory presented. We study the effects of attenuation due to multiple forward scattering on sensing of herring, cod and capelin shoals in an ocean waveguide. A review of developments over past decades in wide-area underwater acoustic sensing of marine life appears in Jagannathan et al. [26]. No attenuation was measured in previously published OAWRS imagery where the acoustic sensing frequencies were off the resonance peak of the shoaling fish, including 
Mid-Atlantic Bight herring [38], Gulf of Maine herring [37] and Lofoten cod [35], as shown in Appendix B. Attenuation resulting in significant reductions in sound pressure level $(\geq 10 \mathrm{~dB})$ is observed in OAWRS imagery presented here of dense herring shoals collected in February 2014 near Ålesund, Norway where swimbladder resonance was near the sensing frequency.

We examine how the shadowing effects of dense fish shoals along the propagation path can be accounted for in OAWRS data to produce accurate estimates of fish areal population density using the waveguide attenuation model of Ratilal and Makris [43]. We also explore methods to predict attenuation from fish in various continental shelf environments and examine how sensing frequency can be adjusted to yield the largest detection ranges.

Attenuation from fish has also been observed in more conventional fisheries acoustics sensing methods that rely on ultrasonic sensors such as downward directed echosounders and sonars [47]. Since ultrasonic fisheries acoustic sensing employs direct paths between sensors and scatterers, modal waveguide propagation effects are not present and simple theoretical formulations derived earlier for multiple scattering in free space are valid [44, 54]. These have traditionally been employed in the analysis of fisheries acoustic data where attenuation due to dense fish groups has been studied [11, 46, 17, 49, 3, 63]. While free space formulations for attenuation from fish are valid for downward directed echosounders, we find that common heuristic approaches that employ free space scattering assumptions for attenuation from fish groups in long-range waveguide propagation can lead to significant errors [59, 13, 32].

\subsection{Materials and Methods}

Evidence of attenuation is observable in OAWRS scattering strength images of herring shoals near Ålesund, Norway. These scattering strength images are generated by beamforming, matched filtering and charting scattered returns and then correcting for source level, transmission loss and areal resolution footprint (Appendix A). Since scattering losses are not incorporated into the transmission loss, attenuation effects are visible when the scattered returns from a distant shoal diminish after an occluding shoal blocks the propagation path (Figure 2-1). In addition, attenuation within a single shoal is observable when the scattered returns from the shoal are strongest at the edge closest to the sensing system, with received sound pressure level decreasing with range. Ambient seafloor scattering in Figure 2-1 is not significantly attenuated behind the occluding shoal. This is because there is a right/left 
ambiguity about the horizontal line-arrayâĂ $Z_{s}$ axis, so if seafloor scattering or ambient noise on one side of the ambiguity is significantly attenuated, the received signal will tend to be dominated by seafloor scattering or ambient noise from the ambiguous side. Ambiguity about the location of fish shoals in OAWRS images was resolved mainly by varying receiver ship heading [19].

Here, we apply the formulation of Ratilal and Makris [43] to attenuation from fish shoals and demonstrate that models that ignore waveguide physics can lead to significant error $(>5$ $\mathrm{dB}$ for a given large shoal) in predicting attenuation in acoustic propagation in an ocean waveguide.

In a waveguide without scatterers, the pressure field at receiver $\boldsymbol{r}$ from source at $\boldsymbol{r}_{\mathbf{0}}$ can be expressed as a sum of modes:

$$
\Psi_{i}\left(\boldsymbol{r} \mid \boldsymbol{r}_{\mathbf{0}}\right)=\sum_{n} \Psi_{i}^{(n)}\left(\boldsymbol{r} \mid \boldsymbol{r}_{\mathbf{0}}\right)
$$

where $\Psi_{i}^{(n)}\left(\boldsymbol{r} \mid \boldsymbol{r}_{\mathbf{0}}\right)$ is the contribution to the field by mode $n . \Psi_{i}^{(n)}\left(\boldsymbol{r} \mid \boldsymbol{r}_{\mathbf{0}}\right)$ can be expressed as

$$
\Psi_{i}^{(n)}\left(\boldsymbol{r} \mid \boldsymbol{r}_{\mathbf{0}}\right)=4 \pi \frac{i}{d\left(z_{0}\right) \sqrt{8 \pi}} e^{-i \pi / 4} u_{n}(z) u_{n}\left(z_{0}\right) \frac{e^{i \xi_{n} \rho}}{\sqrt{\xi_{n} \rho}}
$$

where $\rho$ is the horizontal range, $z$ is water depth, $d\left(z_{0}\right)$ is the medium density at source depth $z_{0}$ and $u_{n}(z)$ is the amplitude of acoustic mode $n$ in the waveguide, which depends on the sound speed profile. The effects of continuous seafloor and sea surface scattering on each mode is incorporated in its complex horizontal wavenumber $\xi_{n}$.

Each $u_{n}(z)$ is normalized such that

$$
\int_{0}^{\infty} \frac{1}{d(z)} u_{n}(z) u_{m}(z) d z=\delta_{n m}
$$

In the formulation developed by Ratilal and Makris [43], the total mean forward field in an acoustic waveguide with scatterers can be expressed as

$$
\left\langle\Psi_{T}\left(\boldsymbol{r} \mid \boldsymbol{r}_{\mathbf{0}}\right)\right\rangle=\sum_{n} \Psi_{i}^{(n)}\left(\boldsymbol{r} \mid \boldsymbol{r}_{\mathbf{0}}\right) e^{i \int_{0}^{\rho} \nu_{n}\left(\rho_{s}\right) d \rho_{s}}
$$

where each dispersion and attenuation coefficient $\nu_{n}$ describes the change in the horizontal wavenumber of mode $n$ as it propagates through the scatterers. Dispersion and attenuation 
coefficients depend on the horizontal wavenumber $\xi_{n}$, the shape of the corresponding mode $u_{n}$, the volume density of the scatterers $n_{V}$ and the scatter function of an individual scatterer $S$. A general formulation for $\nu_{n}$ can be found in Equation (60a) of Reference [43]. Since long-range ocean sensing systems typically operate at low frequencies where the acoustic wavelength is larger than the dimensions of a fish, individual fish will be compact scatterers and the dispersion and attenuation coefficients for fish shoals can be obtained from Equations (19) and (60a) of Reference [43]:

$$
\nu_{n}(\rho)=\int_{0}^{\infty} \frac{2 \pi}{k} \frac{1}{\xi_{n}} \frac{1}{d\left(z_{t}\right)}\left(u_{n}\left(z_{t}\right)\right)^{2}\left\langle s\left(\rho, z_{t}\right)\right\rangle d z_{t}
$$

where $\langle s(\rho, z)\rangle$ is the expected scatter function density of the scatterers. Since the spacing between individual fish is larger than the acoustic wavelength, the fish are incoherent scatterers and the expected scatter function density of a group of fish can be expressed as $\langle s(\rho, z)\rangle=n_{V}(\rho, z)\langle S(\rho, z)\rangle$. Equations for calculating the scatter function $S$ of an individual fish are shown in Appendix C.

The variance of the forward field can be expressed as

$\operatorname{Var}\left(\Psi_{T}\left(\boldsymbol{r} \mid \boldsymbol{r}_{\mathbf{0}}\right)\right)=\sum_{n} \frac{2 \pi}{d^{2}\left(z_{0}\right)} \frac{1}{\left|\xi_{n}\right| \rho}\left|u_{n}\left(z_{0}\right)\right|^{2}\left|u_{n}(z)\right|^{2} e^{-2 \Im\left[\xi_{n} \rho+\int_{0}^{\rho} \nu_{n}\left(\rho_{s}\right) d \rho_{s}\right]}\left(e^{\int_{0}^{\rho} \mu_{n}\left(\rho_{s}\right) d \rho_{s}}-1\right)$

where $\mu_{n}(\rho)$ is the exponential coefficient of modal field variance. A general formulation for $\mu_{n}$ can be found in Equation (94a) of Reference [43]. Since the scatter function of an individual fish is omnidirectional in long-range ocean sensing applications, the exponential coefficient of modal field variance for fish shoals can be obtained from Equations (19), (72) and (94a) of Reference [43]:

$$
\mu_{n}\left(\rho_{s}\right)=\sum_{m} \sqrt{\frac{\rho}{2 \pi \xi_{m} \rho_{s}\left(\rho-\rho_{s}\right)}} \frac{1}{\left|\xi_{m}\right|} \int_{0}^{\infty} \frac{4 \pi^{2}}{k^{2} d^{2}\left(z_{t}\right)}\left|u_{n}\left(z_{t}\right)\right|^{2}\left|u_{m}\left(z_{t}\right)\right|^{2} V_{c}\left(z_{t}\right) \operatorname{Var}\left(s\left(\rho, z_{t}\right)\right) d z_{t}
$$

where the scatter function coherence volume $V_{c}$ quantifies the spatial scale over which the scatter functions of two fish are correlated. The variance of the scatter function density for incoherent scatterers can be obtained from Equations (A23) of Reference [43]:

$$
\operatorname{Var}(s)=\frac{1}{V_{c}} n_{V} \operatorname{Var}(S)
$$


Note that $\mu_{n}$ (Equation (2.7)) is independent of the coherence volume $V_{c}$ since the scatterers are incoherent.

The mean intensity or the second moment of the forward field is the sum of the coherent intensity and incoherent intensity:

$$
\left\langle\left|\Psi_{T}\left(\boldsymbol{r} \mid \boldsymbol{r}_{\mathbf{0}}\right)\right|^{2}\right\rangle=\left|\left\langle\Psi_{T}\left(\boldsymbol{r} \mid \boldsymbol{r}_{\mathbf{0}}\right)\right\rangle\right|^{2}+\operatorname{Var}\left(\Psi_{T}\left(\boldsymbol{r} \mid \boldsymbol{r}_{\mathbf{0}}\right)\right)
$$

Finally, the decrease in sound pressure level due to attenuation in the forward field from scattering can then be expressed as

$$
\Delta S P L=10 \log _{10}\left|\Psi_{i}\left(\boldsymbol{r} \mid \boldsymbol{r}_{\mathbf{0}}\right)\right|^{2}-10 \log _{10}\left(\left\langle\left|\Psi_{T}\left(\boldsymbol{r} \mid \boldsymbol{r}_{\mathbf{0}}\right)\right|^{2}\right\rangle\right)
$$

As derived in Appendix D, the decrease in sound pressure level due to attenuation during two-way propagation to-and-from a uniform distribution of scatterers with mean depth $z_{0}$ and height $H$ positioned at $\boldsymbol{r}_{\boldsymbol{t}}=\left(\boldsymbol{\rho}_{\boldsymbol{t}}, z_{t}\right)$ within resolution footprint $A_{R}\left(\rho_{C}\right)$ can be similarly defined as

$$
\begin{aligned}
\Delta S P L_{2 w a y} & =10 \log _{10}\left(\int_{A_{R}\left(\rho_{C}\right)} \int_{z=z_{0}-H / 2}^{z=z_{0}+H / 2}\left|\Psi_{i}\left(\boldsymbol{r}_{\boldsymbol{t}} \mid \boldsymbol{r}_{\mathbf{0}}\right)\right|^{2}\left|\Psi_{i}\left(\boldsymbol{r} \mid \boldsymbol{r}_{\boldsymbol{t}}\right)\right|^{2} d z_{t} d \boldsymbol{\rho}_{\boldsymbol{t}}^{\mathbf{2}}\right) \\
& -10 \log _{10}\left(\int_{A_{R}\left(\rho_{C}\right)} \int_{z=z_{0}-H / 2}^{z=z_{0}+H / 2}\left\langle\left|\Psi_{T}\left(\boldsymbol{r}_{\boldsymbol{t}} \mid \boldsymbol{r}_{\mathbf{0}}\right)\right|^{2}\right\rangle\left\langle\left|\Psi_{T}\left(\boldsymbol{r} \mid \boldsymbol{r}_{\boldsymbol{t}}\right)\right|^{2}\right\rangle d z_{t} d \boldsymbol{\rho}_{\boldsymbol{t}}^{\mathbf{2}}\right)
\end{aligned}
$$

where $\Psi_{i}\left(\boldsymbol{r}_{\boldsymbol{t}} \mid \boldsymbol{r}_{\mathbf{0}}\right)$ is the incident field from source $\boldsymbol{r}_{\mathbf{0}}$ to target $\boldsymbol{r}_{\boldsymbol{t}}$ and $\Psi_{i}\left(\boldsymbol{r} \mid \boldsymbol{r}_{\boldsymbol{t}}\right)$ is the field scattered from target $\boldsymbol{r}_{\boldsymbol{t}}$ to receiver $\boldsymbol{r}$ (both defined by Equation (2.1)), while $\left\langle\left|\Psi_{T}\left(\boldsymbol{r}_{\boldsymbol{t}} \mid \boldsymbol{r}_{\mathbf{0}}\right)\right|^{2}\right\rangle$ is the total mean intensity from source $\boldsymbol{r}_{\mathbf{0}}$ to target $\boldsymbol{r}_{\boldsymbol{t}}$ and $\left\langle\left|\Psi_{T}\left(\boldsymbol{r} \mid \boldsymbol{r}_{\boldsymbol{t}}\right)\right|^{2}\right\rangle$ is the total mean intensity from target $\boldsymbol{r}_{\boldsymbol{t}}$ to receiver $\boldsymbol{r}$ (both defined by Equation (2.9)).

In the unique case where a fish shoal is uniformly distributed through the water column, we find that attenuation in a waveguide has a form approximately like that found in free space. Specifically, in this case $\langle s(\rho, z)\rangle=n_{V}(\rho)\langle S(\rho)\rangle$ is independent of $z$, so that, with Equation (2.3), Equation (2.5) can be simplified to

$$
\nu_{n}(\rho)=\frac{2 \pi}{k} \frac{1}{\xi_{n}} n_{V}(\rho)\langle S(\rho)\rangle \int_{0}^{\infty} \frac{1}{d\left(z_{t}\right)}\left(u_{n}\left(z_{t}\right)\right)^{2} d z_{t}=\frac{2 \pi}{k} \frac{1}{\xi_{n}} n_{V}(\rho)\langle S(\rho)\rangle
$$


resulting in a total mean field equal to

$$
\left\langle\Psi_{T}\left(\boldsymbol{r} \mid \boldsymbol{r}_{\mathbf{0}}\right)\right\rangle=\sum_{n} \Psi_{i}^{(n)}\left(\boldsymbol{r} \mid \boldsymbol{r}_{\mathbf{0}}\right) e^{i \frac{2 \pi}{k} \frac{1}{\xi_{n}} \int_{0}^{\rho} n_{V}\left(\rho_{s}\right)\left\langle S\left(\rho_{s}\right)\right\rangle d \rho_{s}}
$$

If the horizontal wavenumbers of the propagating modes do not significantly deviate from the wavenumber $\left(\xi_{n} \approx k\right)$, we can further reduce Equation (2.13) using Equation (2.1) to yield

$$
\left\langle\Psi_{T}\left(\boldsymbol{r} \mid \boldsymbol{r}_{\mathbf{0}}\right)\right\rangle=\Psi_{i}\left(\boldsymbol{r} \mid \boldsymbol{r}_{\mathbf{0}}\right) e^{i \frac{2 \pi}{k^{2}} \int_{0}^{\rho} n_{V}\left(\rho_{s}\right)\left\langle S\left(\rho_{s}\right)\right\rangle d \rho_{s}}
$$

which has the same form as the mean field of a free space plane wave propagating through a medium with scatterers, following Rayleigh [45]. If the mean intensity is dominated by the coherent field, attenuation in a waveguide will then follow the free space form of Equation (2.14). We model attenuation in Ålesund waters in a case where a herring shoal uniformly covers the water column. As shown in Figure 2-2, the full waveguide model and the free-space-like factored approximation effectively agree, with a negligible difference between the total attenuation predicted by the two models (less than $0.5 \mathrm{~dB}$ ).

While attenuation from fish in a waveguide approximates free space attenuation when the fish uniformly cover the water column, it can differ significantly when the fish shoal is concentrated at a specific depth. We model attenuation in Ålesund waters in cases where herring are concentrated at the upper, middle and lower water column and we compare the results with a free-space-like factored approximation for attenuation of Equation (2.14) (Figure 2-3). In order to apply a free-space-like factored formulation to concentrated fish shoals, we modify the expected wavenumber change to $\nu_{\text {freespace }}=\left(2 \pi / k^{2}\right)\left(n_{A} / D\right)\langle S\rangle$ where $n_{A}$ is the areal density of the shoal and $D$ is the water depth. This replacement effectively "spreads" a concentrated fish shoal across the water column while retaining the total number of fish. Previous studies have modeled attenuation from fish in a waveguide using this formulation $[7,14]$.

We find that attenuation of each mode depends on the amplitude of the mode at depths where the fish are concentrated and the resulting attenuation will be strongly depthdependent. In the upward-refracting environment modeled here, lower-order acoustic modes concentrated near the sea surface will pass through fish shoals near the seafloor with negligible attenuation and a receiver in the upper water column will measure significantly less attenuation than a receiver near the seafloor. Free-space-like factored formulations that ig- 

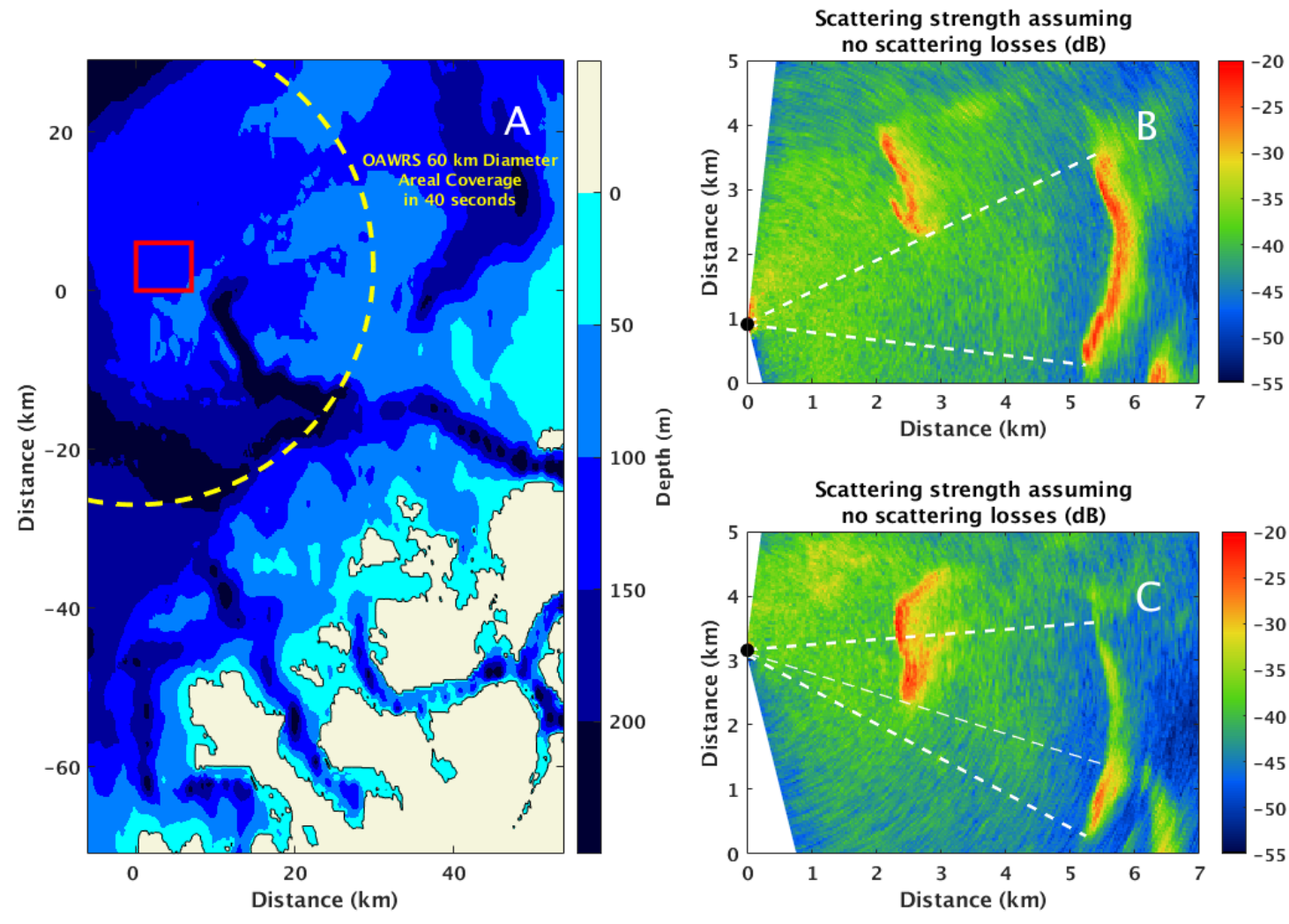

Figure 2-1: Attenuation from multiple forward scattering is observable in Ocean Acoustic Waveguide Remote Sensing (OAWRS) scattering strength images of herring in Alesund waters. Variation in bathymetry near Alesund is shown in (A). The yellow dashed circle shows $60-\mathrm{km}$ diameter OAWRS areal coverage in $40 \mathrm{~s}$. The red rectangular box represents the area investigated here. OAWRS scattering strength images assuming no scattering losses are shown from 21 February 2014 at 04:50:49 (B) and 04:33:19 (C). When the propagation path from the monostatic sensing system (black dot) to the distant shoal (Easting: 5-6 km, Northing: $0-4 \mathrm{~km}$ ) has no occluding shoal, no attenuation is observable, as shown in (B). When an occluding shoal (Easting: 2-3 km, Northing: 2-4 km) is in the propagation path, the scattered returns from the same distant shoal are attenuated, as shown in $(\mathrm{C})$. 

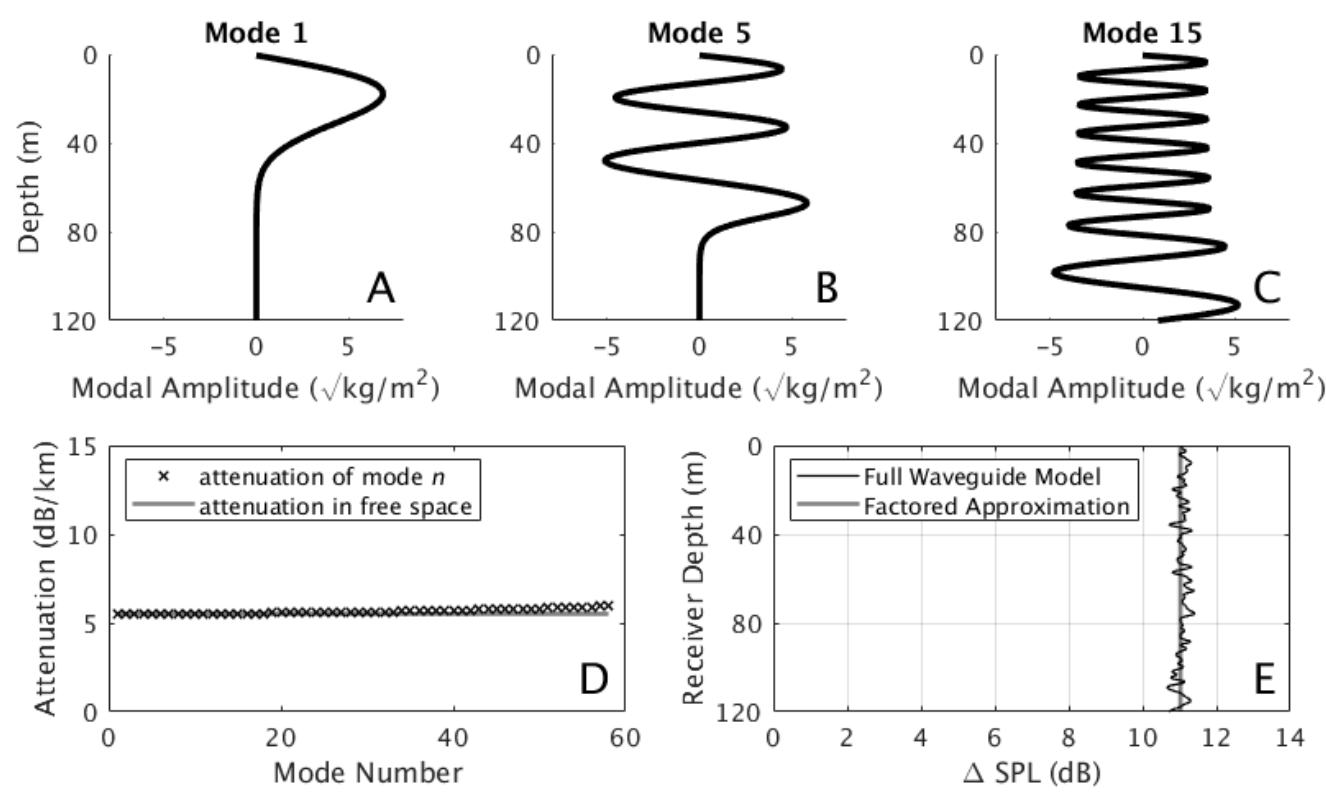

Figure 2-2: When an occluding fish shoal is uniformly distributed through the water column, attenuation in a waveguide has a form similar to that in free space in that the effect of attenuation appears as a single exponential factor in received intensity, as in Equation (2.14). In the example shown here, attenuation of each propagating mode $(\mathrm{A}-\mathrm{C})$ differs from free space attenuation by less than $0.5 \mathrm{~dB} / \mathrm{km}(\mathrm{D})$ and the decrease in sound pressure level due to attenuation $(\triangle S P L)$ matches the free-space-like factored approximation within $0.5 \mathrm{~dB}$ at any receiver depth (E). Here, attenuation during one-way propagation is modeled for Ålesund herring with expected scatter function $\langle S\rangle=-0.03+0.10 i$ in a shoal uniformly distributed between the sea surface and the seafloor $(0-120 \mathrm{~m})$ with width $2 \mathrm{~km}$ and areal population density $2.5 \mathrm{fish} / \mathrm{m}^{2}$ estimated from echosounding data, with a source at depth $60 \mathrm{~m}$ with frequency $955 \mathrm{~Hz}$ and Ålesund sound speed profiles shown in Appendix F. Water density is modeled as $1000 \mathrm{~kg} / \mathrm{m}^{2}$, seafloor density as $1900 \mathrm{~kg} / \mathrm{m}^{2}$ and seafloor sound speed as $1700 \mathrm{~m} / \mathrm{s}$. 
nore these depth-dependent modal attenuation effects can result in significant error $(>5 \mathrm{~dB}$ for a given large shoal).

\subsection{Results and Discussion}

\subsubsection{Consistency between Backscattered Returns and Attenuation}

Here we demonstrate consistency between concurrently measured backscattering and attenuation levels from a single fish shoal using an example of attenuation from herring in Ålesund waters. In Figure 2-1B, there is no occluding shoal between the sensing system and a distant shoal and no attenuation is observed. In Figure $2-1 \mathrm{C}$, the monostatic sensing system is positioned so that an occluding shoal in the propagation path attenuates the scattered returns of the distant shoal. We expect theoretical consistency between the scattering strength of the occluding shoal and the attenuation caused by the occluding shoal, where attenuation is measured as the decrease in scattered returns from the distant shoal after the occluding shoal moves into the propagation path.

The scattering strength and attenuation levels from the occluding shoal are measured using OAWRS data shown in Figure 2-1. The distant shoal and the occluding shoal are segmented by smoothing the scattering strength image using a circular averaging filter of

Figure 2-3 (facing page): When an occluding fish shoal is concentrated at a specific water depth, attenuation will be complicated by waveguide effects so that the free-space-like factored approximation of Equation (2.14) may not be valid. Here, attenuation during one-way propagation is modeled for Ålesund herring shoals uniformly distributed between depths 0-40 m (red), 40-80 m (green) and 80-120 m (blue). Attenuation coefficients associated with given modes and their coupling depends on the specific modal contributions at the depths where the fish group is present $(\mathrm{A}-\mathrm{C})$. Since the environment modeled here is upward refracting, the lowest order modes are most attenuated when the herring occupy the upper water column (D) but they are essentially unattenuated when the herring occupy the lower water column $(\mathrm{H})$. In the case where herring are concentrated near the seafloor, a receiver in the upper water column will receive a strong signal from unattenuated lower order modes, causing the free-space-like factored approximation to overestimate the total attenuation $(\triangle S P L)$ by more than $5 \mathrm{~dB}$ (I). If the herring are concentrated near the sea surface (D-E) or near the center of the water column (F-G), the free-space-like factored approximation is seen to be in error by on the order of $2 \mathrm{~dB}$. Herring are modeled here with expected scatter function $\langle S\rangle=-0.03+0.10 i$ in a shoal with width $2 \mathrm{~km}$ and areal population density $2.5 \mathrm{fish} / \mathrm{m}^{2}$ estimated from echosounding data, with seafloor depth at $120 \mathrm{~m}$, source depth at $60 \mathrm{~m}$ with frequency $955 \mathrm{~Hz}$ and Ålesund sound speed profiles shown in Appendix F. Water density is modeled as $1000 \mathrm{~kg} / \mathrm{m}^{2}$, seafloor density as $1900 \mathrm{~kg} / \mathrm{m}^{2}$ and seafloor sound speed as $1700 \mathrm{~m} / \mathrm{s}$. 

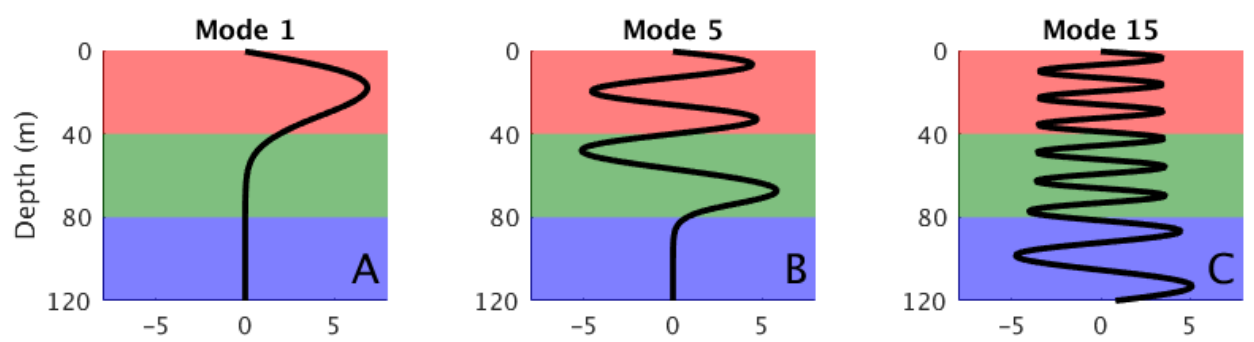

Modal Amplitude $\left(\sqrt{ } \mathrm{kg} / \mathrm{m}^{2}\right)$
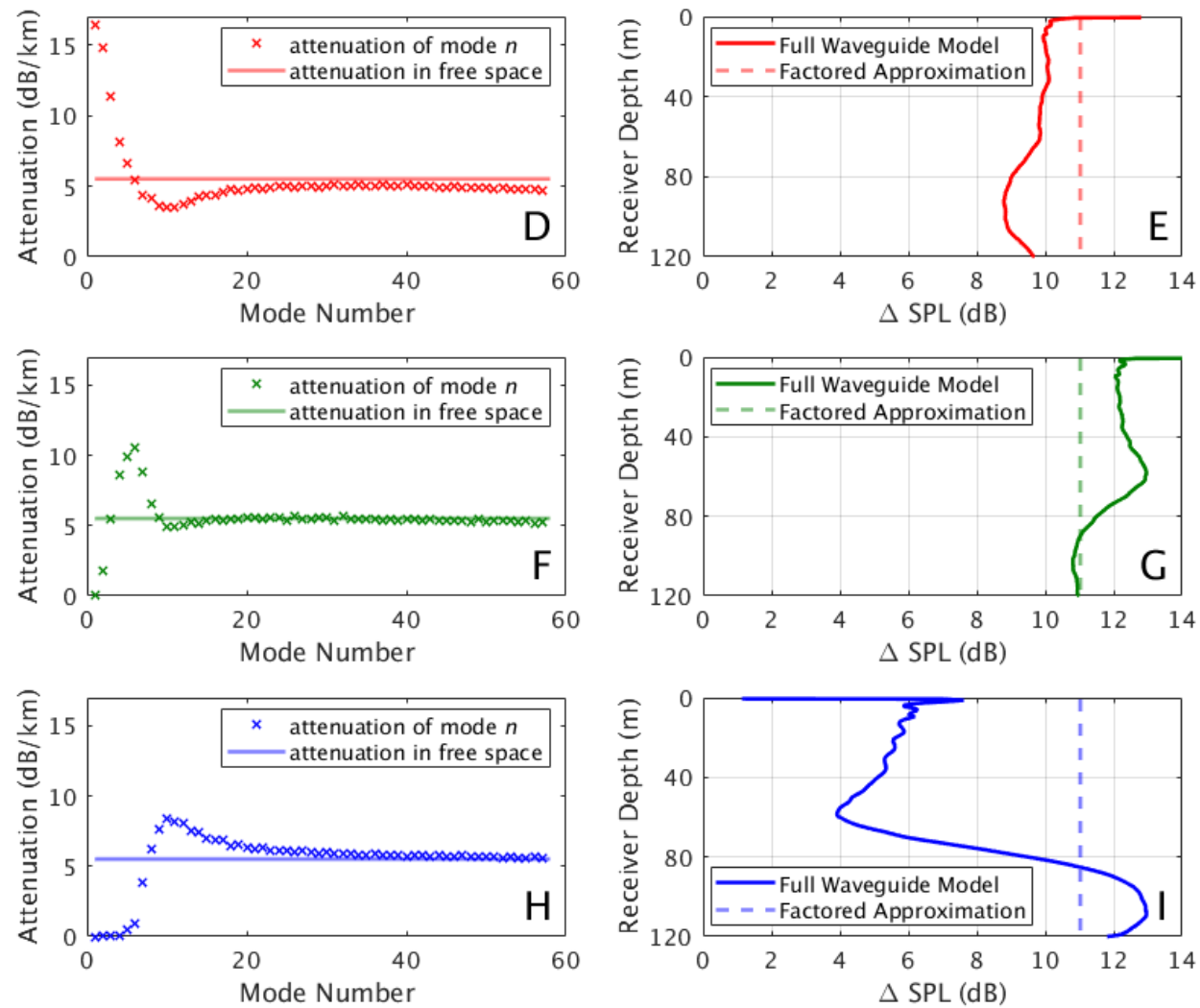
radius $90 \mathrm{~m}$ and selecting regions with scattering strength values greater than $5 \mathrm{~dB}$ above the background seafloor scattering. Total two-way attenuation from the occluding shoal $\left(\triangle S P L_{2 w a y, d a t a}\right)$ is measured as the difference between the scattering strength of the distant shoal before attenuation (Figure 2-1B) and after attenuation (Figure 2-1C). Note that this measurement of $\triangle S P L_{2 w a y, d a t a}$ assumes the areal density and vertical position of the occluding shoal and the distant shoal remain unchanged in the 17 minutes between the observations shown in Figures 2-1B and 2-1C.

When measuring scattering strength of shoals in cases where attenuation is present, care must be taken to exclude the effects of scattering loss within the shoal. We calculate the scattering strength of the occluding shoal $\left(S S_{d a t a}\right)$ by selecting pixels in the shoal with scattering strength values above the 20th percentile, effectively isolating pixels at the edge of the shoal closest to the sensing system, where scattering strength is strongest and attenuation effects are negligible. This analysis is performed for all six operating frequencies used in the 2014 OAWRS experiment.

The modeled scattering strength $S S_{\text {model }}$ of a shoal with mean shoal depth $z_{0}$, shoal thickness $H$ and neutral buoyancy depth $z_{n b}$ at frequency $f$ is calculated [31,61] from

$$
S S_{\text {model }}\left(z_{0}, H, z_{n b}, n_{A}, f\right)=10 \log _{10}\left(\frac{1}{H} \int_{z_{0}-H / 2}^{z_{0}+H / 2} \int_{l}\left|\frac{S\left(z, z_{n b}, l, f\right)}{k}\right|^{2} g(l) d l d z \cdot n_{A}\right)
$$

where $S\left(z, z_{n b}, l, f\right)$ is the far-field scatter function of a single fish, $k$ is the wavenumber in free space, $l$ is the fork length of an individual fish, $g(l)$ is the Gaussian probability density function of the fork length and $z$ is the fish depth. Equations for modeling $S\left(z, z_{n b}, l, f\right)$ are shown in Appendix C. Total two-way attenuation $\Delta S P L_{2 w a y, m o d e l}\left(z_{0}, H, z_{n b}, n_{A}, r_{h}, f\right)$ is modeled from Equation (2.11) using the same five parameters used to calculate $S S_{\text {model }}$ and additionally assumes the horizontal extent of the occluding shoal along the propagation path, $r_{h}$. Note that $r_{h}$ cannot be directly determined from the scattering strength image in Figure 2-1 because attenuation within the occluding shoal masks the scattered returns at the far edge.

The standard deviation of the water depth along the propagation path is less than 5 meters in the region where our measurements are made, which is consistent with the rangeindependent seafloor assumed by the current model. We verify this by using a parabolic equation model [9] to compare transmission between a flat waveguide and a varying-bathymetry 
waveguide using bathymetric data from the region where the experiment took place and we find the difference in transmission to be less than $1 \mathrm{~dB}$.

Modeled scattering strength and attenuation values are simultaneously matched with the data using the maximum likelihood estimate of fish shoal parameters $z_{0}, H, z_{n b}, n_{A}$ and $r_{h}$. Since the acoustic field can be described as a circular complex Gaussian random variable (CCGR) and the time-bandwidth product of the acoustic measurements $\mu=$ (1 second) $(50 \mathrm{~Hz}) \gg 1$, intensity measurements in the logarithmic domain such as $S S$ and $\triangle S P L_{2 w a y}$ can be well-approximated as Gaussian random variables with variance independent of the mean $[33,34]$. Since scattering strength and attenuation are dependent on many of the same parameters $\left(z_{0}, H, z_{n b}\right.$ and $\left.n_{A}\right)$, we treat $S S$ and $\triangle S P L_{2 w a y}$ as correlated random variables. We then estimate fish shoal parameters $\hat{\boldsymbol{\theta}}=\left[\hat{z_{0}}, \hat{H}, \hat{z}_{n b}, \hat{n}_{A}, \hat{r}_{h}\right]$ by maximizing the log-likelihood function $\ell(\boldsymbol{\theta})$ assuming $S S$ and $\triangle S P L_{2 \text { way }}$ are correlated Gaussian random variables with variance independent of the mean, as derived in Appendix E:

$$
\begin{aligned}
& \ell(\boldsymbol{\theta})=\sum_{i=1}^{N_{f}}\left(-\frac{\left(S S_{\text {model }}\left(\boldsymbol{\theta}, f_{i}\right)-S S_{\text {data }}\left(f_{i}\right)\right)^{2}}{\sigma_{S S}\left(f_{i}\right)^{2}}-\frac{\left(\Delta S P L_{2 \text { way,model }}\left(\boldsymbol{\theta}, f_{i}\right)-\Delta S P L_{2 \text { way }, \text { data }}\left(f_{i}\right)\right)^{2}}{\sigma_{\Delta S P L}\left(f_{i}\right)^{2}}\right. \\
& \left.+2 \sigma_{S S, \Delta S P L}\left[\frac{\left(S S_{\text {model }}\left(\boldsymbol{\theta}, f_{i}\right)-S S_{\text {data }}\left(f_{i}\right)\right)}{\sigma_{S S}\left(f_{i}\right)^{2}} \times \frac{\left(\Delta S P L_{2 \text { way,model }}\left(\boldsymbol{\theta}, f_{i}\right)-\Delta S P L_{2 \text { way,data }}\left(f_{i}\right)\right)}{\sigma_{\Delta S P L}\left(f_{i}\right)^{2}}\right]\right)
\end{aligned}
$$

where $\sigma_{S S}\left(f_{i}\right)^{2}$ is the variance of the measured scattering strength in $\mathrm{dB}$ at frequency $f_{i}$, $\sigma_{\triangle S P L}\left(f_{i}\right)^{2}$ is the variance of the measured attenuation in $\mathrm{dB}$ at frequency $f_{i}, \rho_{S S, \triangle S P L}\left(f_{i}\right)$ is the sample covariance between measured scattering strength and attenuation at frequency $f_{i}$ and $N_{f}$ is the number of frequencies where $S S$ and $\triangle S P L_{2 w a y}$ are measured. The maximum of the log-likelihood function is determined through an exhaustive grid search across the five unknown parameters $\left(z_{0}, H, z_{n b}, n_{A}, r_{h}\right)$. The ranges of these parameters are determined such that the herring shoal physically stays within the water column $(H<D$ and $H / 2<z_{0}<D-H / 2$, where $D$ is the water depth) and the areal number density is physically realistic $\left(n_{A}<10\right.$ fish $/ \mathrm{m}^{2}$, as determined by echosounder observations).

When fish shoal parameters are inverted by maximizing the log-likelihood function, the modeled scattering strength and attenuation values match measured values within a standard deviation, as shown in Figure 2-4. This consistency between scattering strength and attenuation demonstrates that the waveguide attenuation model can accurately predict at- 

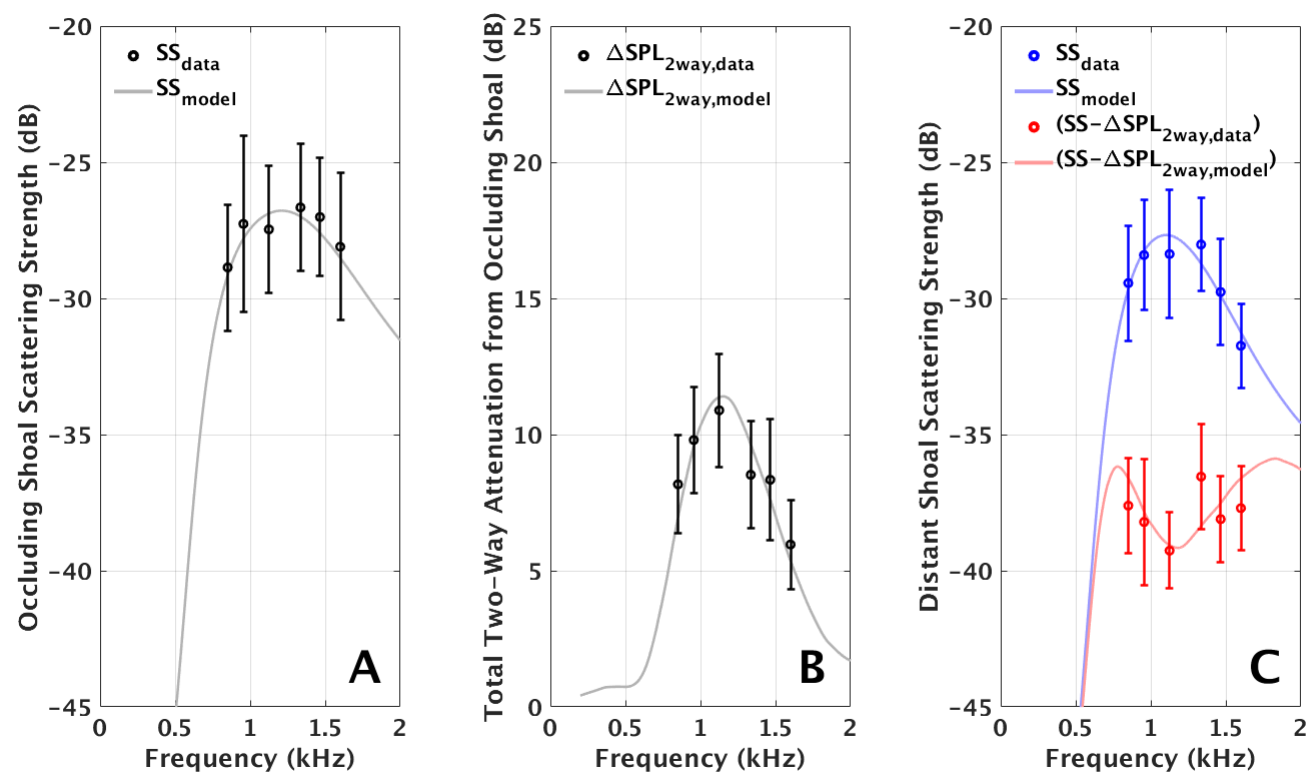

Figure 2-4: Theoretical consistency is demonstrated between concurrently measured backscattering strength (A) and attenuation due to multiple forward scattering (B) from the occluding shoal shown in Figure 2-1. Attenuation is measured as the decrease in scattered returns from a distant shoal after the occluding shoal moves into the propagation path, that is the difference between the red and blue data in (C). Modeled scattering strength and total attenuation (grey lines in A and B, respectively) assume the same fish shoal parameters and fit the data within one standard deviation. Scattering strength and attenuation of the occluding shoal (A and B) are modeled using the following estimated parameters: mean shoal depth $z_{0}=60 \mathrm{~m}$, shoal thickness $H=40 \mathrm{~m}$, neutral buoyancy depth $z_{n b}=0 \mathrm{~m}$, areal number density $n_{A}=2.4 \mathrm{fish} / \mathrm{m}^{2}$ and horizontal extent $r_{h}=1.1 \mathrm{~km}$. Scattering strength of the distant shoal (blue line in $\mathrm{C}$ ) is modeled using the following estimated parameters: mean shoal depth $z_{0}=85 \mathrm{~m}$, shoal thickness $H=50 \mathrm{~m}$, neutral buoyancy depth $z_{n b}=10$ $\mathrm{m}$ and areal number density $n_{A}=1.1 \mathrm{fish} / \mathrm{m}^{2}$.

tenuation levels in ocean sensing applications.

\subsubsection{Attenuation Prediction and Frequency Selection}

Using the waveguide attenuation model, we demonstrate how sensing frequency can be adjusted to yield the largest detection range for acoustic sensing of fish shoals in various continental shelf environments. Scattering strength and attenuation are modeled for each fish species and environment and we search for the sensing frequency that maximizes scattering strength uncorrected for two-way attenuation from fish $\left(S S-\triangle S P L_{2 \text { way }}\right)$, which in turn maximizes the detection range of the sensing system. Here $S S$ is modeled using Equation (2.15) and $\triangle S P L_{2 w a y}$ is modeled using Equation (2.11). 
Table 2.1: Environmental parameters used in Figure 2-5.

$\begin{array}{ccccccc}\text { Environment/Species } & \begin{array}{c}\text { Areal } \\ \text { Density } \\ \left(\mathbf{f i s h} / \mathbf{m}^{\mathbf{2}}\right)\end{array} & \begin{array}{c}\text { Water } \\ \text { Depth } \\ (\mathbf{m})\end{array} & \begin{array}{c}\text { Shoal } \\ \text { Depth } \\ (\mathbf{m})\end{array} & \begin{array}{c}\text { Shoal Vertical } \\ \text { Thickness } \\ (\mathbf{m})\end{array} & \begin{array}{c}\text { Neutral } \\ \text { Buoyancy Depth } \\ (\mathbf{m})\end{array} & \begin{array}{c}\text { Mean Fish } \\ \text { Length } \\ (\mathbf{c m})\end{array} \\ \text { Gulf of Maine herring } & 2^{\mathrm{a}} & 200^{\mathrm{a}} & 150^{\mathrm{a}} & 30^{\mathrm{a}} & 82^{\mathrm{a}} & 24^{\mathrm{a}} \\ \text { Alesund herring } & 2.5^{\mathrm{b}} & 120^{\mathrm{b}} & 60^{\mathrm{b}} & 40^{\mathrm{b}} & 0^{\mathrm{c}} & 34^{\mathrm{d}} \\ \text { Lofoten cod } & 0.05^{\mathrm{e}} & 100^{\mathrm{e}} & 75^{\mathrm{e}} & 50^{\mathrm{e}} & 75^{\mathrm{e}} & 83^{\mathrm{e}} \\ \text { Finnmark capelin } & 10^{\mathrm{b}} & 300^{\mathrm{b}} & 30^{\mathrm{b}} & 40^{\mathrm{b}} & 10^{\mathrm{f}} & 17^{\mathrm{d}}\end{array}$

a [19], ${ }^{b}$ Measured from echogram data collected during the experiment, ${ }^{\mathrm{c}}$ Figure 2-4, ${ }^{\mathrm{d}}$ Measured from trawl samples collected during the experiment, ${ }^{\mathrm{e}}[35],{ }^{\mathrm{f}}[26]$.

We model the scattering strength uncorrected for attenuation of fish shoals in four environments: herring in the Gulf of Maine, herring in Ålesund waters, cod in Lofoten waters and capelin in Finnmark waters (Figure 2-5) using environmental and species-specific parameters determined from synoptic echogram data and trawl samples (Table 2.1). We observe a tradeoff between 1) frequencies near swimbladder resonance, where backscattering is highest but attenuation can weaken the signal and 2) frequencies off-resonance, where attenuation is negligible but backscattering is weaker. When the horizontal extent of fish along the propagation path is less than $1 \mathrm{~km}$, attenuation is less of a concern and frequencies near fish swimbladder resonance are optimal. As the horizontal extent of high-density shoals in the propagation path increases, attenuation will severely limit sensing near resonance and off-resonance frequencies will become more favorable.

The predictions of the waveguide attenuation model shown in Figure 2-5 are in agreement with attenuation levels observed in previous OAWRS experiments. Significant attenuation from Ålesund herring $(\triangle S P L>10 \mathrm{~dB})$ is predicted at the sensing frequencies used in the 2014 experiment, which is consistent with observations from that experiment. The absence of attenuation from Gulf of Maine herring in 2006 and Lofoten cod in 2014 can be explained by off-resonance sensing frequencies. Attenuation from Finnmark capelin was less than 2.5 $\mathrm{dB}$ even at resonance because the horizontal extent of observed capelin shoals was not large enough to produce significant attenuation $(<0.25 \mathrm{~km})$.

This analysis shows the waveguide attenuation model can be used to determine the conditions for significant attenuation and select sensing frequencies that maximize detection range. For example, attenuation from Ålesund herring can be avoided by choosing a sensing frequency above or below the swimbladder resonance of the fish shoals. The results of our model are specific to lower-frequency acoustic sensing, where the dimensions of a fish swimbladder are smaller than the acoustic wavelength. 

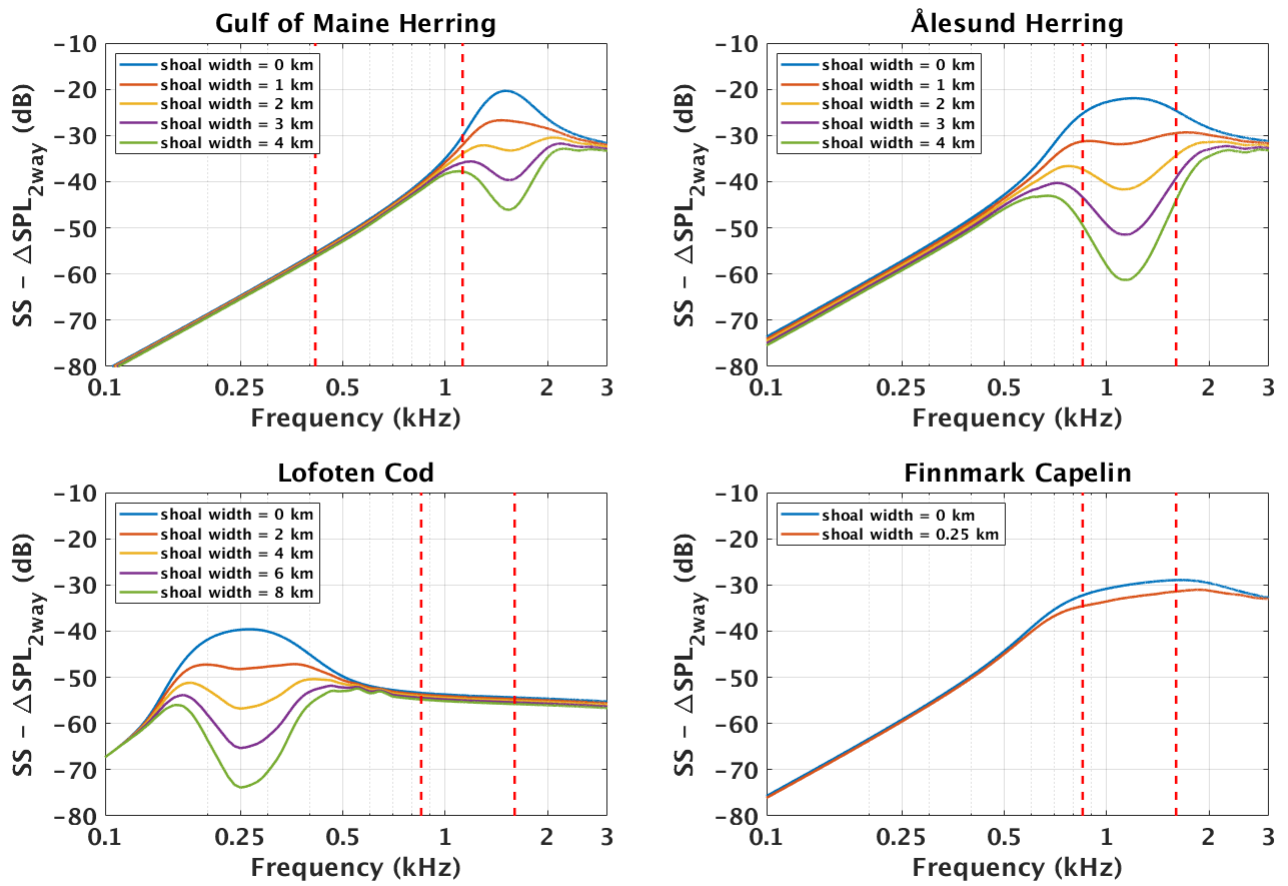

Figure 2-5: The most favorable acoustic frequency for sensing in an ocean environment can be determined by maximizing the scattering strength uncorrected for two-way attenuation from fish $\left(S S-\triangle S P L_{2 w a y}\right)$ of a target. Here, $\left(S S-\triangle S P L_{2 w a y}\right)$ is modeled for fish shoals in four continental shelf environments: herring in the Gulf of Maine, herring in Ålesund waters, cod in Lofoten waters and capelin in Finnmark waters. The magnitude of attenuation depends on the width of the shoals along the propagation path between the sensing system and the target. When the shoal width along the propagation path is sufficiently small, attenuation will be negligible and the most favorable sensing frequency will be near swimbladder resonance. As the shoal width along the propagation path increases, there will be a tradeoff between frequencies near resonance where scattering strength is highest and off-resonance frequencies where attenuation is lowest. Red dotted lines designate the sensing frequency range of OAWRS experiments in each region. Physical parameters used for modeling each environment are shown in Table 2.1. 


\subsubsection{Estimating Scattering Strength with Attenuation}

We developed a method for accounting for attenuation in scattering strength measurements using the waveguide attenuation model. A scattering strength image uncorrected for scattering losses is generated from OAWRS data using the method described in Appendix A. In regions where scattering is dominated by fish, each scattering strength pixel can be corrected according to the following equation:

$$
S S\left(r_{0}, \theta_{0}\right)=\widetilde{S S}\left(r_{0}, \theta_{0}\right)+\Delta S P L_{2 w a y}\left(r_{0}, \theta_{0}\right)
$$

where $\left(r_{0}, \theta_{0}\right)$ are polar coordinates defined with respect to the monostatic sensing system,

$S S$ is the true scattering strength, $\widetilde{S S}$ is the scattering strength uncorrected for scattering losses and $\triangle S P L_{2 w a y}$ is two-way attenuation due to fish calculated from Equation (2.11). As shown in Equation (2.5), $\triangle S P L_{2 w a y}\left(r_{0}, \theta_{0}\right)$ will depend on the volume density of the fish between the sensing system and the target, $n_{V}\left(r \in\left[0, r_{0}\right], \theta_{0}\right)$, which can be calculated at each pixel as a function of $S S$ according [19] to

$$
n_{V}(r, \theta)=\frac{n_{A}(r, \theta)}{H}=\frac{1}{H} 10^{(S S(r, \theta)-T S) / 10}
$$

where $n_{A}$ is the areal density of the fish, $H$ is the shoal thickness and $T S$ is the target strength of an individual fish (modeled in Appendix C). As a result, each calculation of scattering strength, $S S\left(r_{0}, \theta_{0}\right)$, depends on the scattering strength at pixels between the sensing system and the target, $S S\left(r \in\left[0, r_{0}\right], \theta_{0}\right)$, so Equation (2.17) must be iteratively applied to pixels closest to the sensing system before correcting pixels further out.

Using this method, we modify the OAWRS scattering strength image shown in Figure 2$6 \mathrm{~A}$ to include scattering losses. We designate regions where scattering is dominated by fish by smoothing the scattering strength image using a circular averaging filter of radius $90 \mathrm{~m}$ and selecting regions with scattering strength values greater than $5 \mathrm{~dB}$ above the background seafloor scattering. Corrections are made to pixels within these regions assuming the fish are uniformly distributed between depths $40 \mathrm{~m}$ and $80 \mathrm{~m}$ with neutral buoyancy depth $0 \mathrm{~m}$, as determined by the parameter inversion in Equation (2.16).

After modifying the scattering strength image to include scattering losses, shadowing effects caused by attenuation are no longer visible (Figure 2-6B). The scattering strength 
of the fish groups are comparatively uniform in space and are not biased by the horizontal extent of fish in the propagation path. It is important to note that scattering loss corrections cannot be made in cases where the attenuated signal is masked by ambient noise or background seafloor scattering. As a result, detection range limitations caused by attenuation from fish can only be improved by selecting a proper sensing frequency, as discussed in Section 2.3.2.

\subsection{Discussion}

We experimentally and theoretically demonstrate how propagation through vast and dense shoals of swimbladder-bearing fish can attenuate acoustic signals and reduce detection range for long-range sensing in the ocean as a consequence of scattering and absorption by the fish. We modeled attenuation from fish shoals in continental-shelf environments using a normalmode based formulation derived from first principles for acoustic propagation through inhomogeneities in a waveguide. In previous experiments, either the attenuation from fish in the shoal or the scattering cross sections of fish in the shoal were measured but not both, making it impossible to directly confirm a theoretical prediction on attenuation through the shoal. Here, both measurements have been made and they experimentally confirm the waveguide theory presented. We show that common heuristic approaches that employ free space scattering assumptions for attenuation from fish groups can lead to significant error ( $>5 \mathrm{~dB}$ for a given large shoal) for applications involving long-range waveguide propagation and scattering. This same basic theory applies to fish without swimbladders, except there will be no resonance effects and attenuation is expected to be increasing with frequency as in Rayleigh-Born scattering.

\subsection{Conclusions}

We experimentally show with wide-area sensing over $360^{\circ}$ in the horizontal and ranges spanning many tens of kilometers that a single large fish shoal can significantly occlude acoustic sensing over entire sectors spanning more than $30^{\circ}$ with corresponding decreases in detection ranges by roughly an order of magnitude. Such blockages can comprise significant impediments to underwater acoustic remote sensing and surveillance of underwater vehicles, marine life and geophysical phenomena as well as underwater communication. We apply a rigorous 

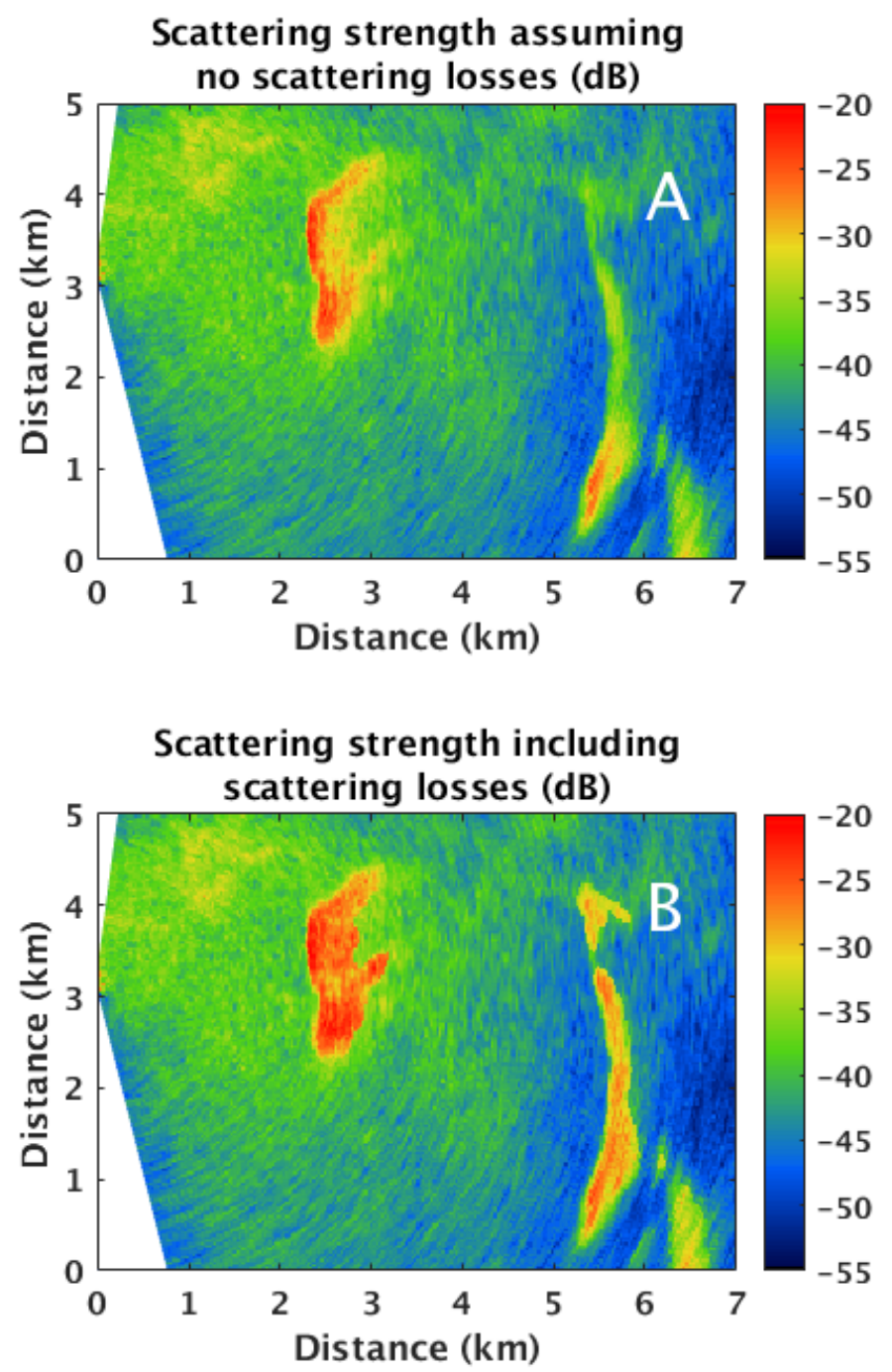

Figure 2-6: The waveguide attenuation model can be used to account for scattering losses in acoustic backscattering data. Here, scattering strength images of herring shoals are shown from 04:33:19 on 21 February 2014 with (A) no scattering losses included and (B) scattering losses included. After accounting for scattering losses, the scattering strength of both the occluding shoal (Easting: 2-3 km, Northing: 2-4 km) and the distant shoal (Easting: 5$6 \mathrm{~km}$, Northing: 0-4 km) are comparatively uniform in space and are not biased by the horizontal extent of fish in the propagation path. Scattering losses are calculated using the following parameters: mean shoal depth $z_{0}=60 \mathrm{~m}$, shoal height $H=40 \mathrm{~m}$ and neutral buoyancy depth $z_{n b}=0 \mathrm{~m}$. 
formulation for modeling multiple forward scattering through inhomogeneities in an ocean waveguide to model attenuation through fish shoals in a continental shelf environment. The approach is found to accurately predict attenuation observed in Ocean Acoustic Waveguide Remote Sensing measurements over a variety of species and environments, including herring in the Georges Bank and the Nordic Seas, as well as cod and capelin in the Nordic Seas. The approach can be applied to both active and passive waveguide propagation and sensing through fish. We show that previous attenuation models that ignore waveguide physics can lead to sound pressure level predictions that are in error by at least an order of magnitude in many practical scenarios. We show that for sensing through fish shoals at frequencies near the peak swimbladder resonance of the fish, attenuation can adversely affect sensing within and beyond the shoal. In these cases, we find that the detection range of sensing systems can be maximized by choosing sensing frequencies off swimbladder resonance where attenuation is negligible. We also show how waveguide attenuation modeling can be used to more accurately estimate scattering amplitudes and fish population densities in regions where shadowing from attenuation is present. 


\section{Chapter 3}

\section{Quantification of Wide-Area}

Norwegian Spring-Spawning Herring

Population Density with Ocean

\section{Acoustic Waveguide Remote Sensing}

\subsection{Introduction}

Norwegian spring-spawning herring is a critical economic resource for multiple nations in the North Atlantic. Since 2013, disagreements over the shifting spatial distribution of this herring stock have prevented these nations from negotiating quota sharing agreements, resulting in a combined catch exceeding the recommended limit by more than $30 \%$ in the past three years [39]. It is therefore important to accurately measure population size and spatial distribution for Norwegian herring over ecosystem scales. Oceanic fish groups, however, occupy vast undersea areas that are difficult to sample without significant aliasing in space and time with conventional survey methods [10, 29].

Here we demonstrate the ability of Ocean Acoustic Waveguide Remote Sensing (OAWRS) to instantaneously image Norwegian herring shoals over more than one thousand square kilometers in spawning grounds near Ålesund, Norway. Wide-area OAWRS scattering strength maps are generated by correcting transmissions for source level and areal resolution footprint, as well as transmission losses from spreading and seafloor attenuation [37, 38, 26, 19, 2]. 
During the day, the OAWRS system reveals small, dispersed groups of herring in the Ålesund spawning grounds with dimensions that are typically less than $1 \mathrm{~km}$. After nautical sunset, the formation of numerous dense herring groups is observed, corresponding with a significant range-dependent decay in OAWRS imagery even after making typical corrections for spreading loss and seafloor attenuation. This range-dependent decay can be explained by acoustic attenuation through the dense herring groups. Such attenuation requires exceptionally high fish population densities over significant portions portions of the water column and extended ranges, and so was not observed previously in OAWRS fish sensing including Mid-Atlantic Bight herring [38], Gulf of Maine herring [26] and Lofoten cod [35]. Reductions in ambient noise level after nautical sunset can be similarly explained by attenuation from the herring groups.

The range-dependent decay in OAWRS transmissions is corrected with a theoretical formulation that has been previously shown to be consistent with experimental measurements of attenuation from fish in a waveguide environment $[43,15]$. After applying the attenuation correction, wide-area population density maps can be generated even during dense shoaling activity. Several large herring groups are observed after sunset with dimensions of several kilometers and population densities of up to roughly $5 \mathrm{fish} / \mathrm{m}^{2}$, which is consistent with echosounder measurements during this time.

Measured reductions in sensing range due to attenuation from fish are shown to be theoretically predictable from waveguide scattering theory and observed population densities. Theoretical predictions of sensing range are confirmed using measurements of OAWRS sensing range in the presence of herring in Ålesund spawning grounds, capelin in Finnmark spawning grounds, and cod in Lofoten spawning spawning grounds. This formulation can be used to predict the performance of active sensing systems using historical surveys of fish population density in the relevant region, as well as fluctuations in sensing range using known diel and annual variations in population density. Such sensing range reductions may also limit the ability of vocalizing marine mammals to echolocate in regions with dense fish shoals $[62,27]$.

Spatial-temporal inhomogenieties in wide-area herring distributions observed by OAWRS indicate that significant errors are expected in sparse temporal-spatial line transect surveys through this region during spawning. Equivalent echosounder measurements of herring population, for example, are estimated by sampling 2014 spatially continuous OAWRS wide- 
area population density data with the sparse line-transects of a 2001 herring survey in the same region. The corresponding sparse line-transect survey population estimates range from 0.5 to 2.5 times the population found using the entire OAWRS population density data for the region. This suggests that OAWRS can be a valuable tool for surveying spawning herring groups in conjunction with conventional line-transect methods in this region.

\subsection{Wide-Area OAWRS population density measurements with- out significant attenuation from herring}

Ocean Acoustic Waveguide Remote Sensing (OAWRS) is used to instantaneously monitor Norwegian herring spawning grounds over more than one thousand square kilometers off the coast of Ålesund, Norway (Figure 2-1A). OAWRS sound pressure level maps are generated by beamforming, matched filtering and charting scattered returns [37, 38, 26, 19, 2]. Scattering strength levels are determined by correcting sound pressure level maps for source level, areal resolution footprint, spreading loss and seafloor attenuation [37, 38, 26, 19] (Appendix A).

Approximately one hour before nautical sunset, the OAWRS system reveals small, dispersed groups of herring with dimensions that are typically less than $1 \mathrm{~km}$ (Figure 3-1A), and there are few herring groups observed in echosounder measurements from the research vessel towing the OAWRS system (Figure 3-1B). Scattering strength is converted to areal population density by calibration with local in situ measurements of population density obtained from vertical echosounder measurements (Appendix C), and the dispersed herring groups are found to have population densities on the order of $0.3 \mathrm{fish} / \mathrm{m}^{2}$ (Figure 3-2). During this time, the median sensing range over azimuth for the OAWRS system is approximately 20 $\mathrm{km}$, where sensing range is defined as the minimum range where scattered returns from the environment fall within the detection threshold of the ambient noise (Appendix H). Attempts to measure scattering strength beyond the sensing range limit where ambient noise dominates will not lead to an actual scattering strength but to a quantity that increases with range since transmission loss corrections are being applied to ambient noise with relatively constant mean intensity over time unless it is dominated by a particular nearby ship or sound source. 


\subsection{Correcting for attenuation from herring in wide-area OAWRS population density maps}

On the same day and in the same region as the daylight measurements of Figures 3-1 and 3-2, there is a significant range-dependent decay in the intensity of OAWRS transmissions after sunset, even after making the same standard corrections as in Figure 3-1 for source level, areal resolution footprint, spreading loss and seafloor attenuation (Figure 3-3). This rangedependent decay can be explained by acoustic attenuation from dense herring groups forming at sunset, which are observed in both OAWRS imagery and echosounder measurements from the research vessel towing the OAWRS system.

OAWRS images are corrected for attenuation from fish scattering using a theoretical formulation that has been previously shown to be consistent with experimental measurements of attenuation from fish in a waveguide $[43,15]$. The theoretical decay due to fish attenuation depends on the average population density of fish within the sensing region, which is determined for each OAWRS transmission by modeling scattering strength uncorrected for losses from attenuation ("fish-attenuated scattering strength") and performing a leastsquares fit with measurements (Figure 3-4). Fish-attenuated scattering strength is measured by correcting OAWRS transmissions for source level, areal resolution footprint, spreading

Figure 3-1 (facing page): Wide area OAWRS scattering strength map approximately one hour before nautical sunset (2014 February 20, 17:55:49). Scattering strength is measured by correcting OAWRS transmissions for source level, areal resolution footprint, spreading loss and seafloor attenuation. The sensing range (thick white line) is approximately $20 \mathrm{~km}$. Denser fish groups appear at scattering strength levels above the labeled "Fish Shoaling Threshold" corresponding to the critical population density where herring groups were found to form $\left(0.2 \mathrm{fish} / \mathrm{m}^{2}\right)$ [37, 38]. Below the labeled "Seafloor Scattering Threshold" (0.05 fish $/ \mathrm{m}^{2}$ ) fish groups are not reliably distinguishable from seafloor scattering. Regions between the seafloor scattering threshold and the fish shoaling threshold may include contributions from both dispersed fish groups and seafloor scattering. Regions beyond the sensing region (thick white line) are mostly dominated by ambient noise where $S P L-S L-T L A$ will increase with range, since transmission loss corrections are being applied to constant ambient noise. During this time there are few herring groups observed in echosounder measurements from the research vessel towing the OAWRS system (B), consistent with the OAWRS imagery in (A). The solid cyan line in (A) shows the path of the research vessel corresponding to the echogram shown, and the white dot shows the position of the monostatic OAWRS system. The cyan dotted line in (B) corresponds to the time when this OAWRS transmission was recorded. Thin white lines designate bathymetric contours. 

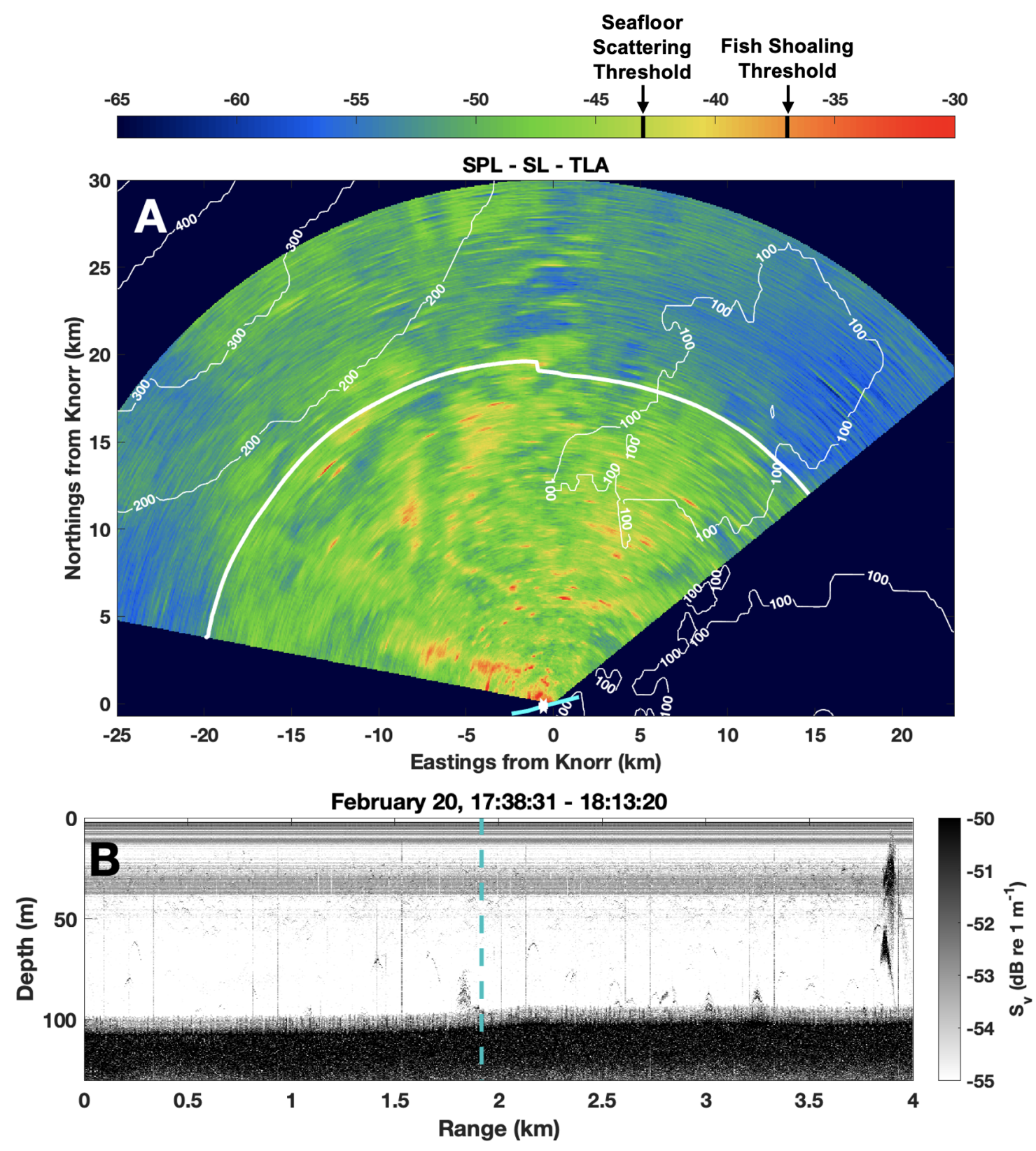


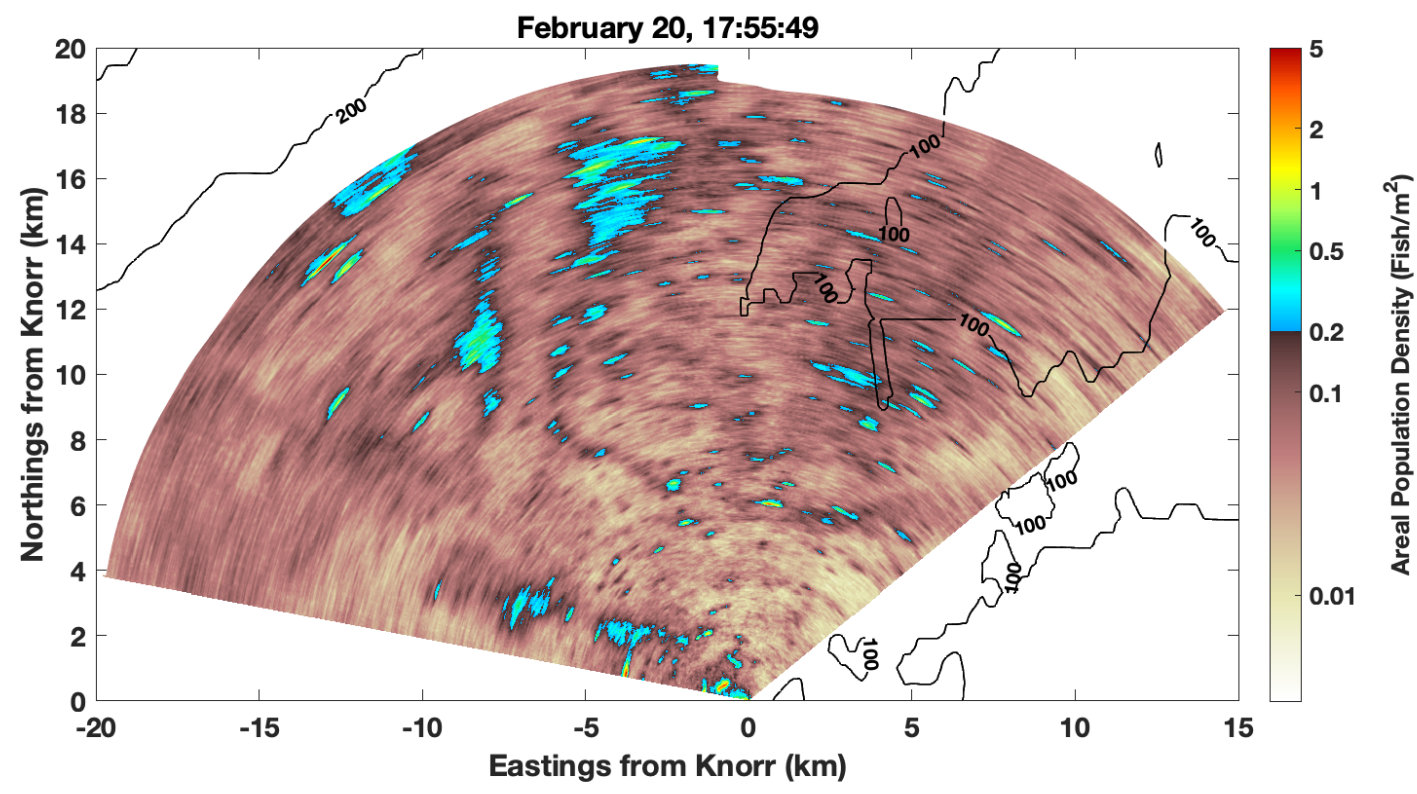

Figure 3-2: OAWRS population density map of Norwegian spring spawning herring approximately one hour before nautical sunset (2014 February 20, 17:55:49). Herring shoals with population density above the $0.2 \mathrm{fish} / \mathrm{m}^{2}$ critical density where shoals were found to form [37, 38], are relatively sparsely distributed before sunset and have densities consistent with echosounder measurements during this time (Figure L-1). The average population density measured here is $0.07 \mathrm{fish} / \mathrm{m}^{2}$, where regions below the minimum detectable herring density $0.05 \mathrm{fish} / \mathrm{m}^{2}$ are not included in the measurement. Black lines designate bathymetric contours.

Figure 3-3 (facing page): Two hours after nautical sunset, several large, dense herring shoals are observed within the OAWRS sensing region, as well as a significant range-dependent decay caused by acoustic attenuation from the herring groups. An OAWRS transmission corrected for source level, areal resolution footprint, spreading loss and seafloor attenuation from 2014 February 20, 21:13:19 is shown in (A), where a significant range-dependent decay is observed. Many larger herring groups are seen to have formed after sunset in the OAWRS imagery, each often spanning a few kilometers, as confirmed by echosounder measurements from the research vessel towing the OAWRS system (B). The position of the OAWRS system is nearly identical to Figure 3-1, however the sensing range (thick white line) is reduced by $20 \%$ due to attenuation from fish. While herring groups can be visually observed in the scattering strength map shown, it is difficult to determine which regions are dominated by scattering from fish or seafloor without correcting the OAWRS imagery for attenuation from fish. The solid cyan line in (A) shows the path of the research vessel corresponding to the echogram shown, and white dot shows the position of the monostatic OAWRS system. The cyan dotted line in (B) corresponds to the time when this OAWRS transmission was recorded. Thin white lines designate bathymetric contours. 

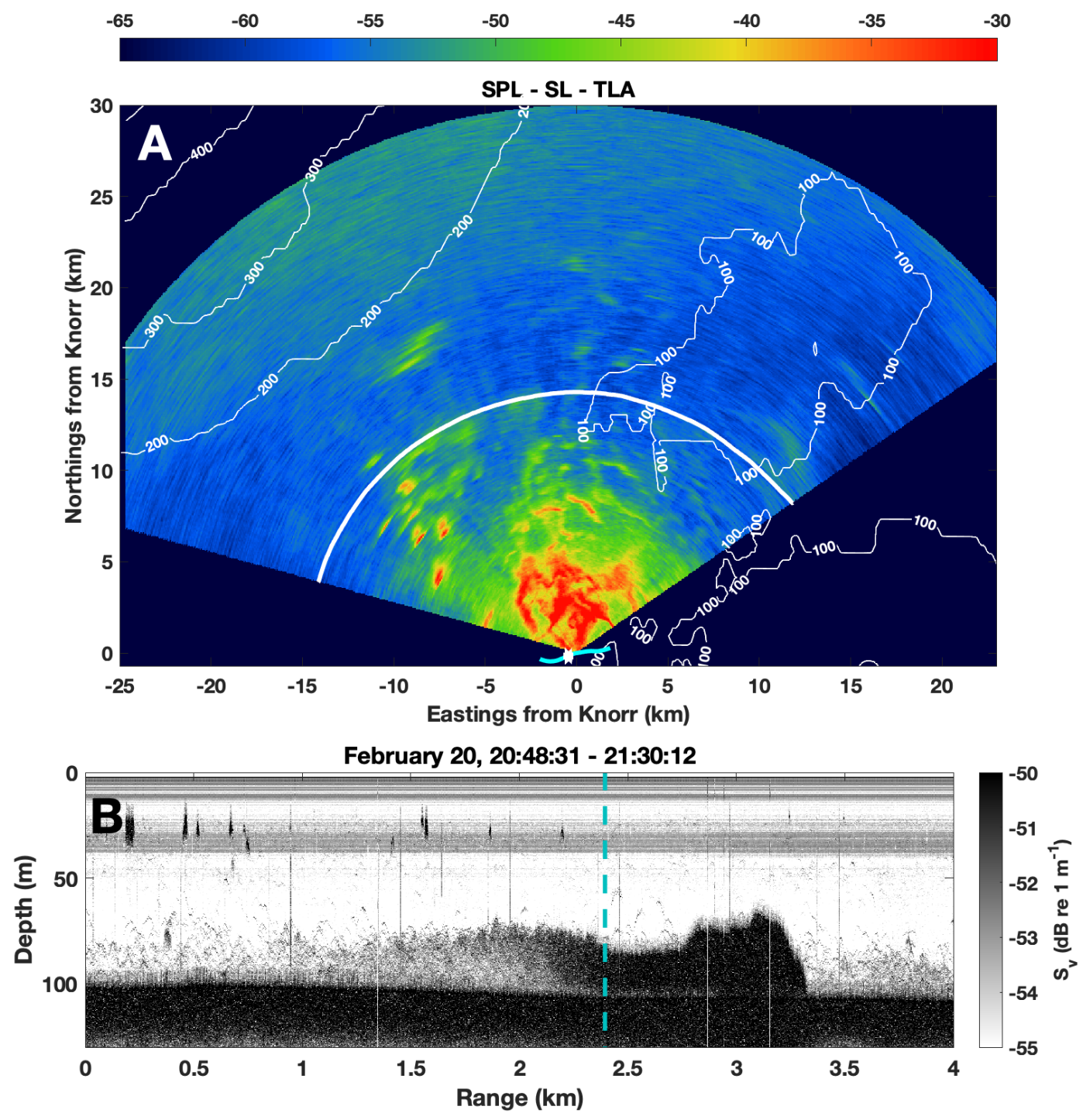
Table 3.1: Environmental parameters used in Figure 3-4

$\begin{array}{cccccc}\text { Environment/Species } & \begin{array}{c}\text { Water } \\ \text { Depth } \\ (\mathbf{m})\end{array} & \begin{array}{c}\text { Shoal } \\ \text { Depth } \\ (\mathbf{m})\end{array} & \begin{array}{c}\text { Shoal Vertical } \\ \text { Thickness } \\ (\mathbf{m})\end{array} & \begin{array}{c}\text { Neutral } \\ \text { Buoyancy Depth } \\ (\mathbf{m})\end{array} & \begin{array}{c}\text { Mean Fish } \\ \text { Length } \\ (\mathbf{c m})\end{array} \\ \text { Ålesund herring } & 100^{\mathrm{a}} & 65^{\mathrm{b}} & 70^{\mathrm{b}} & 3^{\mathrm{c}} & 34^{\mathrm{a}} \\ \text { Lofoten cod } & 100^{\mathrm{a}} & 75^{\mathrm{a}} & 50^{\mathrm{a}} & 75^{\mathrm{a}} & 83^{\mathrm{a}} \\ \text { Finnmark capelin } & 300^{\mathrm{a}} & 30^{\mathrm{a}} & 40^{\mathrm{a}} & 10^{\mathrm{a}} & 17^{\mathrm{a}}\end{array}$

${ }^{\mathrm{a}}[15]{ }^{\mathrm{b}}$ Appendix L ${ }^{\mathrm{c}}$ Appendix G

loss and seafloor attenuation and averaging across azimuthal angle. To generate wide-area OAWRS population density images, the further step of correcting for range-dependent decay due to fish scattering is applied using a combination of empirical data and modeling (Appendix I). Multiple large herring groups are observed after sunset with dimensions of several kilometers and population densities of up to roughly $5 \mathrm{fish} / \mathrm{m}^{2}$, which is consistent with echosounder measurements during this time (Figure L-2).

Reductions in ambient noise level are also observed after nautical sunset, and they can be similarly explained by attenuation from herring groups (Figure 3-7). No significant changes in measured wind speed after sunset are present to possibly provide another mechanism

Figure 3-4 (facing page): The range-dependent decay in OAWRS transmissions after nautical sunset is found to be consistent with theoretical predictions for attenuation from fish, which can be used to correct OAWRS images for fish attenuation. The theoretical decay due to fish depends on the average population density within the sensing region, which is determined for each OAWRS transmission by modeling scattering strength uncorrected for losses from fish attenuation ("fish-attenuated scattering strength") and performing a least-squares fit with measurements (A-C). Fish-attenuated scattering strength is measured for each OAWRS transmission by correcting for source level, areal resolution footprint, spreading loss and seafloor attenuation and averaging across azimuthal angle (Appendix I). Before nautical sunset, measurements of fish-attenuated scattering strength do not significantly change with range (black line in A). After nautical sunset, an increase in fish-attenuated scattering strength is observed at ranges below $6 \mathrm{~km}$, as well as a significant decay with range (black lines in B-C). Fish-attenuated scattering strength is modeled assuming a horizontally uniform distribution of herring with species-specific parameters shown in Table 3.1. The average areal number density of herring within the sensing range of the OAWRS system is determined by performing a least-squares fit between measured and modeled fish-attenuated scattering strength (red lines in A-C). The log-likelihood function is calculated by integrating the weighted difference squared across range in polar coordinates (Equation (A3), where measurements at longer ranges are weighted more heavily since they correspond to a larger area. Average population density significantly increases in the hours after nautical sunset, from approximately $0.08 \mathrm{fish} / \mathrm{m}^{2}$ before 18:30 to nearly $0.3 \mathrm{fish} / \mathrm{m}^{2}$ after 21:00 (D). 

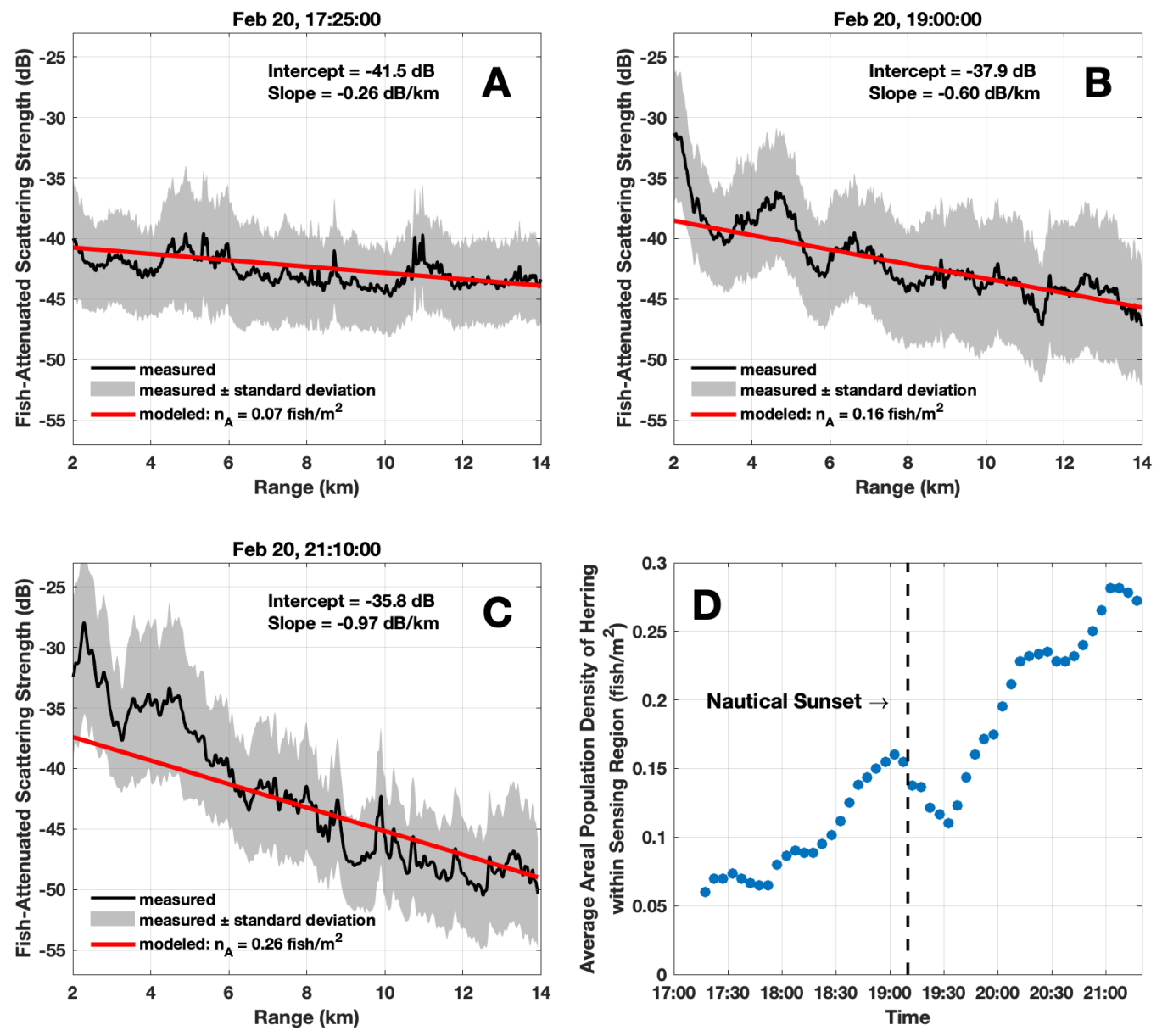


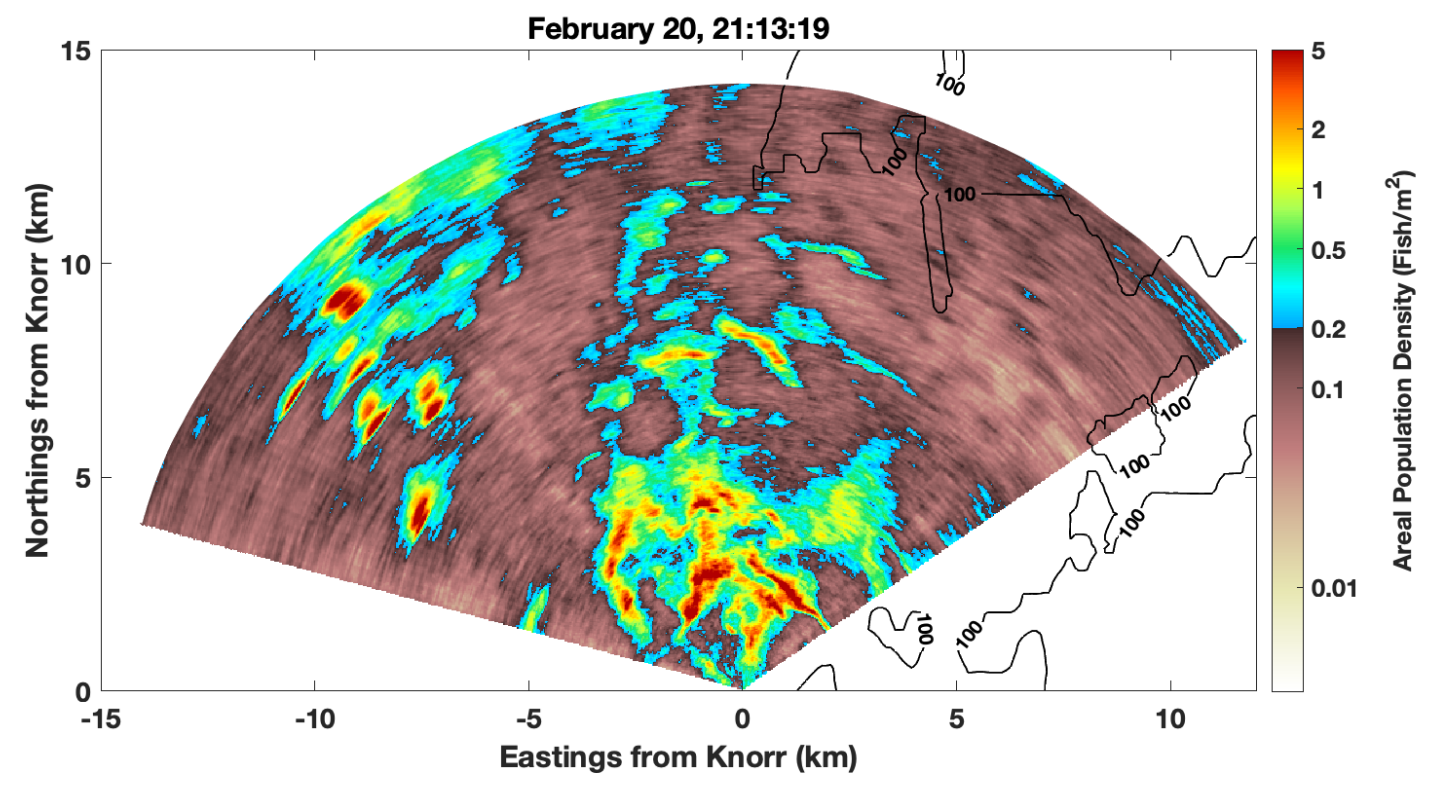

Figure 3-5: OAWRS population density map of Norwegian spring spawning herring approximately two hours after nautical sunset (2014 February 20, 21:13:19). Here a correction has been applied to account for attenuation from fish, where the modeled decay is estimated from the average areal density within the sensing region calculated in Figure 3-4. Multiple large herring groups are observed with dimensions of several kilometers and population densities of up to roughly $5 \mathrm{fish} / \mathrm{m}^{2}$, which is consistent with echosounder measurements during this time (Figure L-2). The average population density measured here is $0.29 \mathrm{fish} / \mathrm{m}^{2}$, where regions below the minimum detectable herring density $0.05 \mathrm{fish} / \mathrm{m}^{2}$ are not included in the measurement. The color scale is chosen so that the transition from brown to blue occurs at $0.2 \mathrm{fish} / \mathrm{m}^{2}$, which is the critical population density at which large herring shoals were found to form [37, 38]. Black lines designate bathymetric contours.

(Figure M-1). Reductions in ambient noise level at sunset are found to be consistent with theoretical reductions in ambient noise based on the increase in herring population density observed by OAWRS (Appendix J).

\subsection{Predicting sensing range reductions due to attenuation from fish}

The range-dependent decay in OAWRS transmissions due to attenuation from fish leads to significant reductions in sensing range (Figure 3-6). Thirty minutes before nautical sunset, the average fish population density within the sensing region is below $0.1 \mathrm{fish} / \mathrm{m}^{2}$ and the sensing range remains approximately constant $(20 \mathrm{~km})$. In the two hours following nautical 
sunset, fish population density increases to nearly $0.3 \mathrm{fish} / \mathrm{m}^{2}$, corresponding with reductions in sensing range of $20 \%$.

Measured reductions in sensing range are found to be theoretically predictable from waveguide scattering theory and observed population densities. Sensing range predictions depend on the average density of fish within the sensing region as well as the source power of the sensing system and ambient noise level (Appendix K). Sensing range is predicted for both Ålesund herring and Finnmark capelin, and predictions are in agreement with OAWRS measurements in both environments (Figure 3-8). Sensing range predictions for Lofoten cod exceed the maximum possible range that could be recorded in the 50 second recording window that was used in this region (Figure 3-8C).

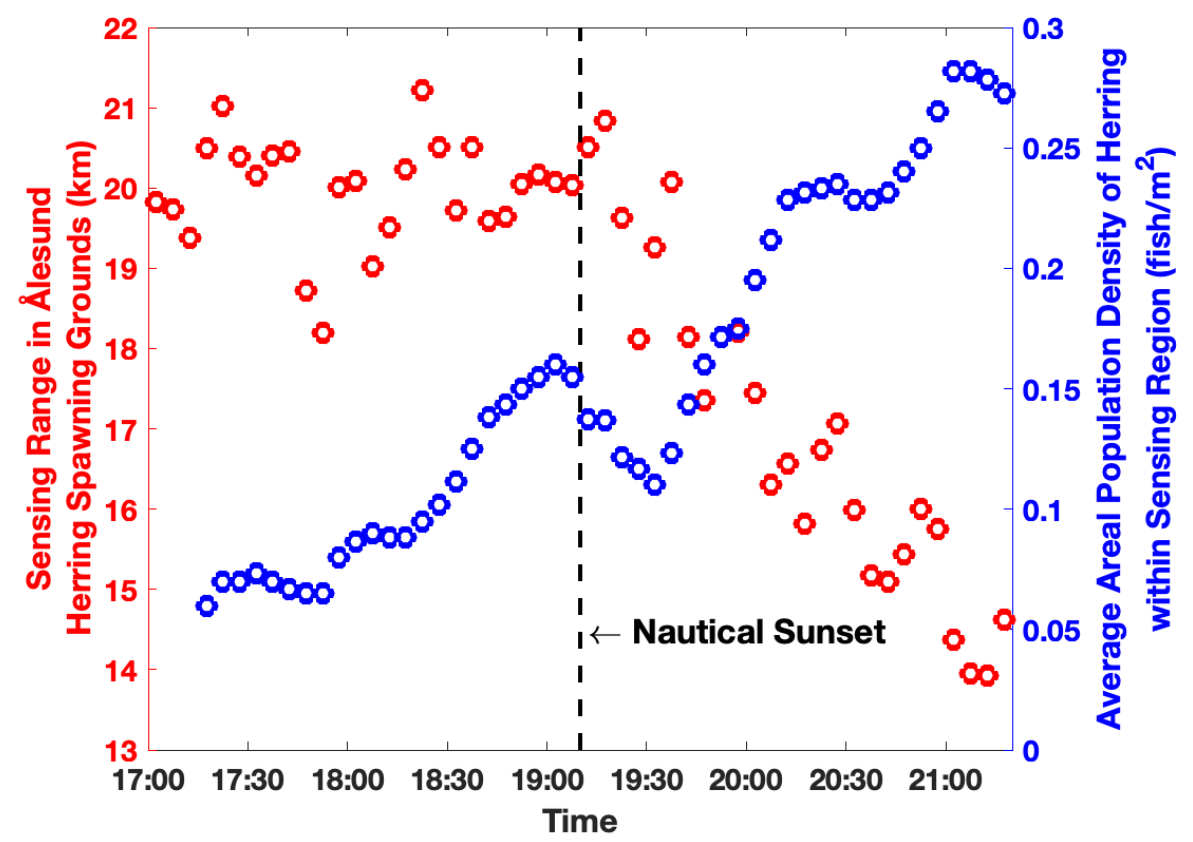

Figure 3-6: The increase in herring population density at nautical sunset is correlated with a reduction in sensing range for the OAWRS system. Before 18:00, the average areal population density of herring (blue data) is approximately 0.07 fish $/ \mathrm{m}^{2}$, and the sensing range (red data) remains stable at approximately $20 \mathrm{~km}$. Over the course of the two hours following nautical sunset, the average areal density of herring within the sensing region increases to nearly $0.3 \mathrm{fish} / \mathrm{m}^{2}$, corresponding with a $20 \%$ reduction in sensing range. 

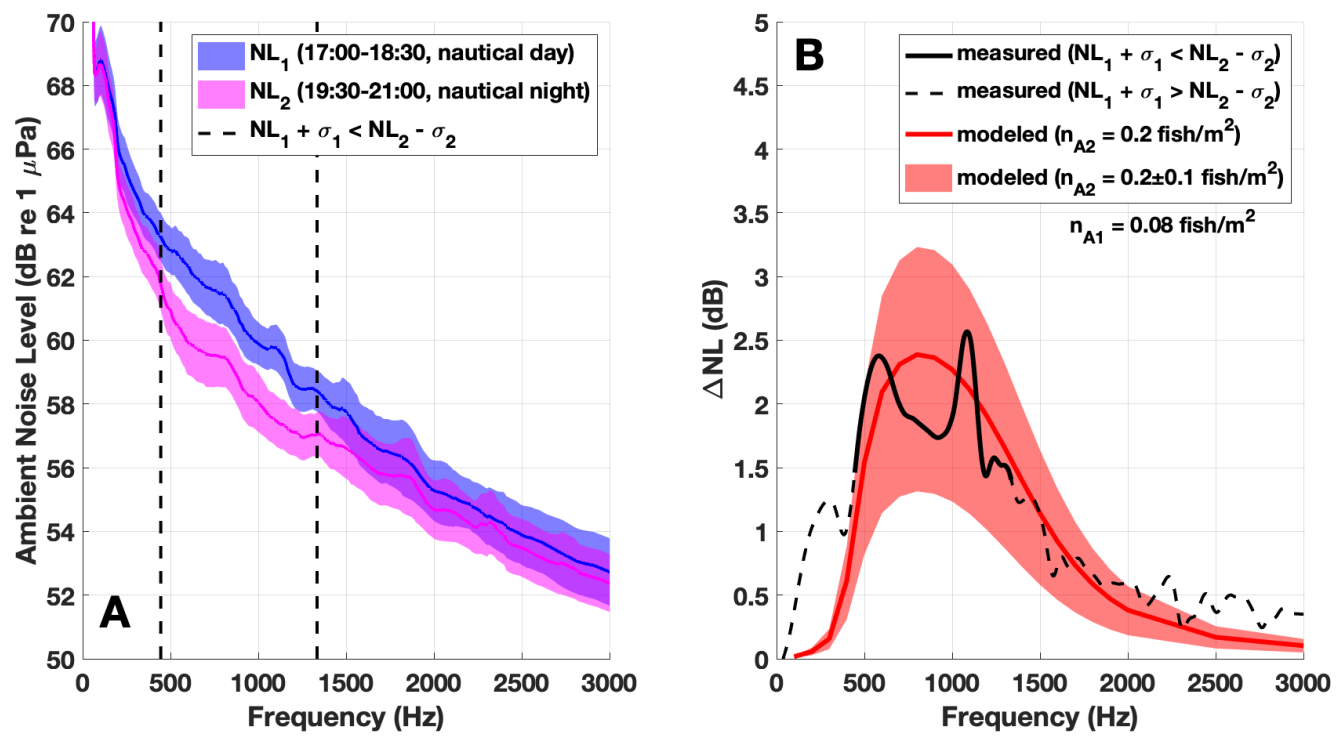

Figure 3-7: Reductions in ambient noise level observed after nautical sunset are consistent with predicted reductions in ambient noise due to attenuation from fish. The decrease in ambient noise $(\Delta N L$, black data in B) is measured as the difference between the mean ambient noise level before nautical sunset $N L_{1}$ (17:00-18:30, blue data in A) and after nautical sunset $N L_{2}$ (19:30-21:00, magenta data in A). Ambient noise measurements before and after nautical sunset are separated by more than a standard deviation $\left(N L_{1}+\sigma_{1}<N L_{2}-\sigma_{2}\right)$ for frequencies within in an $890 \mathrm{~Hz}$ band near the swimbladder resonance peak of the herring (black dotted lines in A). The solid black line in (B) denotes measured $\Delta N L$ for frequencies within this band, and the dotted black lines in (B) denote measured $\Delta N L$ for frequencies outside of this band. The modeled reduction in ambient noise from fish depends on the average areal population density before nautical sunset $\left(n_{A 1}\right)$ and after sunset $\left(n_{A 2}\right)$, which are respectively determined from the mean of OAWRS measurements of average population density from 17:00-18:30 and 19:30-21:00 (Figure 3-4D). Solid lines in (A) show the mean ambient noise level within each time frame, and shaded patches denote the standard deviation. The dominant source of ambient noise at the frequencies studied is surface agitation from wind [58].

\subsection{Spatial undersampling in echosounder surveys}

Spatial inhomogenieties in wide-area herring distributions seen synoptically in OAWRS imagery can be spatially undersampled in typical echosounder surveys, which can result in significant overestimation or underestimation of the fish population in the survey region. Equivalent echosounder estimates of herring population density, for example, are made by sampling 2014 spatially continuous OAWRS wide-area population density data (Figures 3-2, 3-5, and 3-9) with the sparse line-transects of a 2001 herring survey in the same region [48]. The total population estimate from line-transect sampling is then calculated by multiplying 

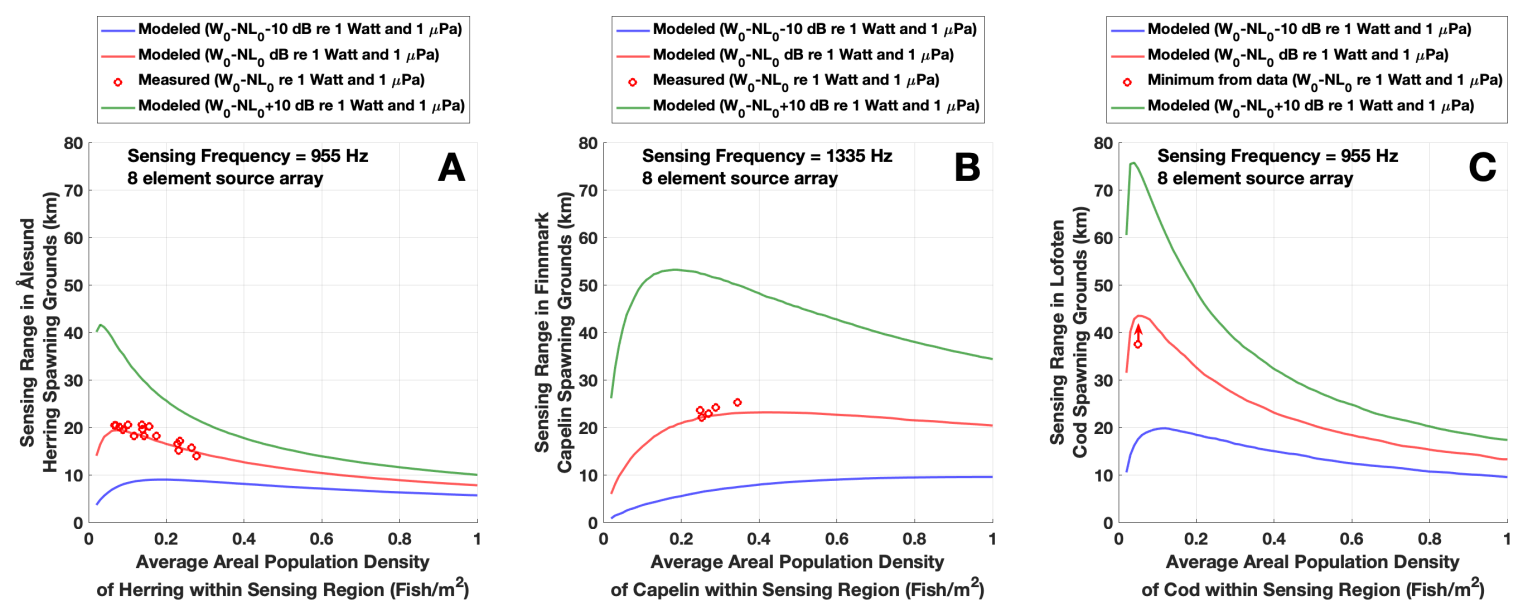

Figure 3-8: Sensing range predictions are shown for multiple environments where fish groups are known to shoal, and theoretical predictions are found to be in agreement with sensing range measurements for the OAWRS system. Expected sensing range is shown in the presence of (A) herring shoals in Ålesund waters, (B) capelin shoals in Finnmark waters, and (C) cod shoals in Lofoten waters. Sensing range predictions depend on the input source power of the sensing system and the ambient noise level. Sensing range is modeled in each environment for $W_{0}-N L_{0} \pm 10 \mathrm{~dB}$ re $1 \mu \mathrm{Pa}$, where $W_{0}$ is the experimental input source power used and $N L_{0}$ is the experimental noise level. Measured sensing range for the OAWRS system in the presence of Alesund herring and Finnmark capelin (red dots in A and B) are shown to be in agreement with expected sensing range at the relevant $W_{0}-N L_{0}$ values (red lines in $\mathrm{A}$ and $\mathrm{B}$ ). Physical parameters used for modeling sensing range for Finnmark capelin and Lofoten cod are shown in Table 1 of [15]. Sensing range predictions for Lofoten cod exceed the maximum possible range that could be recorded in the 50 second recording window that was used in this region (red dot in $\mathrm{C}$ ). Here the received signal is assumed to be dominated by scattering from fish.

the average population density along the transect with a specified survey area (Figure 311A). Resulting population estimates in this area range from 0.5 to 2.5 times the population found using the entire OAWRS population density data for the region (Figure 3-11B). Widearea spatial population density maps made with OAWRS can be used in conjunction with conventional line-transect survey methods to provide better estimates herring population and spatial distribution.

\subsection{Discussion}

It is experimentally shown that Ocean Acoustic Waveguide Remote Sensing (OAWRS) can be used to instantaneously measure wide-area fish population density, even in environments 

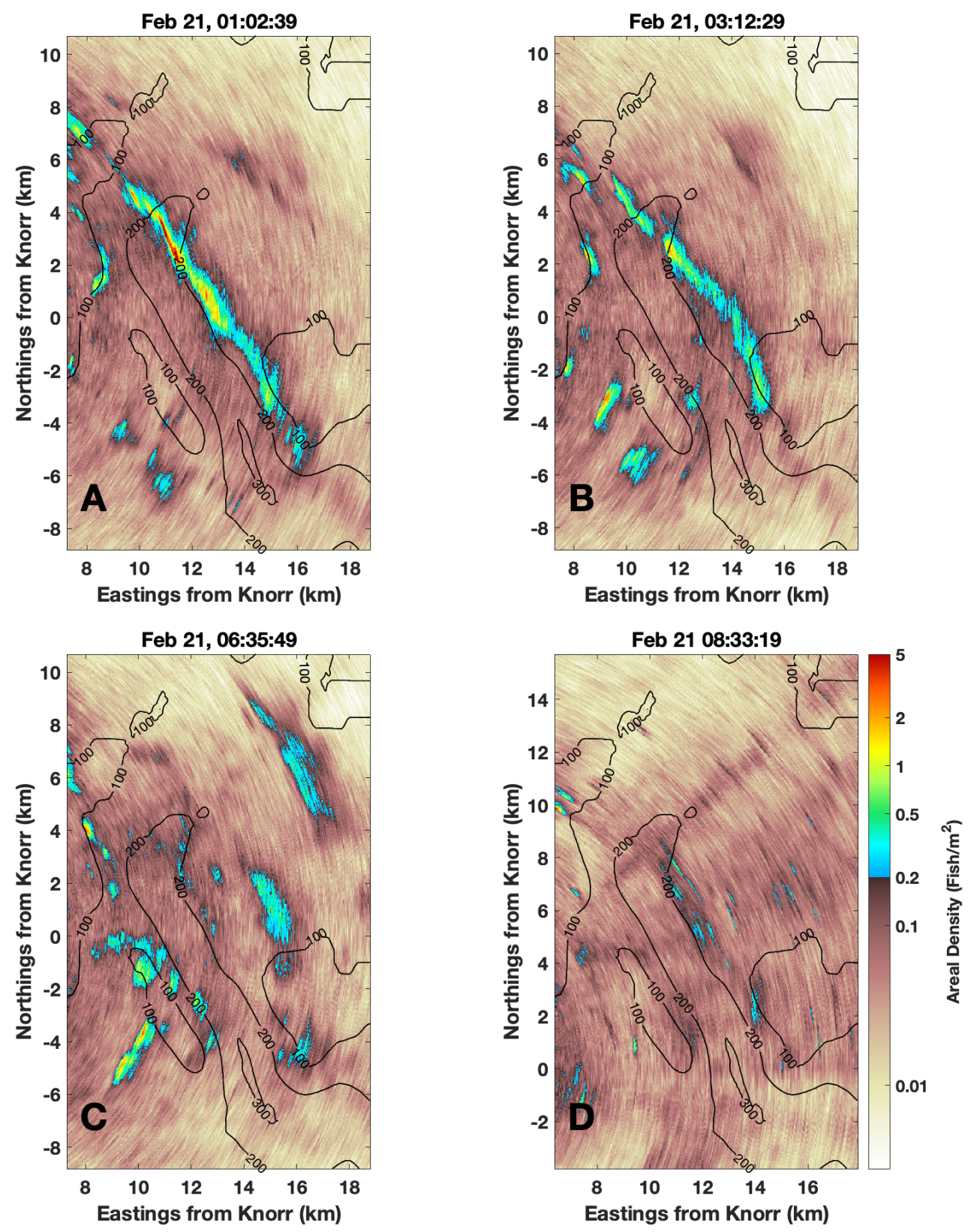

Figure 3-9: Sample instantaneous OAWRS images of herring shoals near Ålesund trench, a historic spawning ground, over a seven hour period (2014 February 21 (01:02:39-08:33:19). On February 21 before nautical sunrise (6:30), a large, elongated herring shoal is observed on the northeastern edge of the trench with a total population on the order of 4 million (A-B). Five minutes after nautical sunrise, this large shoal begins to fragment (C). Two hours after nautical sunrise, the shoal has dissipated (D). The measured population size within the survey box for each OAWRS image shown here is in Figure 3-10. The color scale is chosen so that the transition from brown to blue occurs at $0.2 \mathrm{fish} / \mathrm{m}^{2}$, which is the critical population density at which large herring shoals were found to form $[37,38]$. 


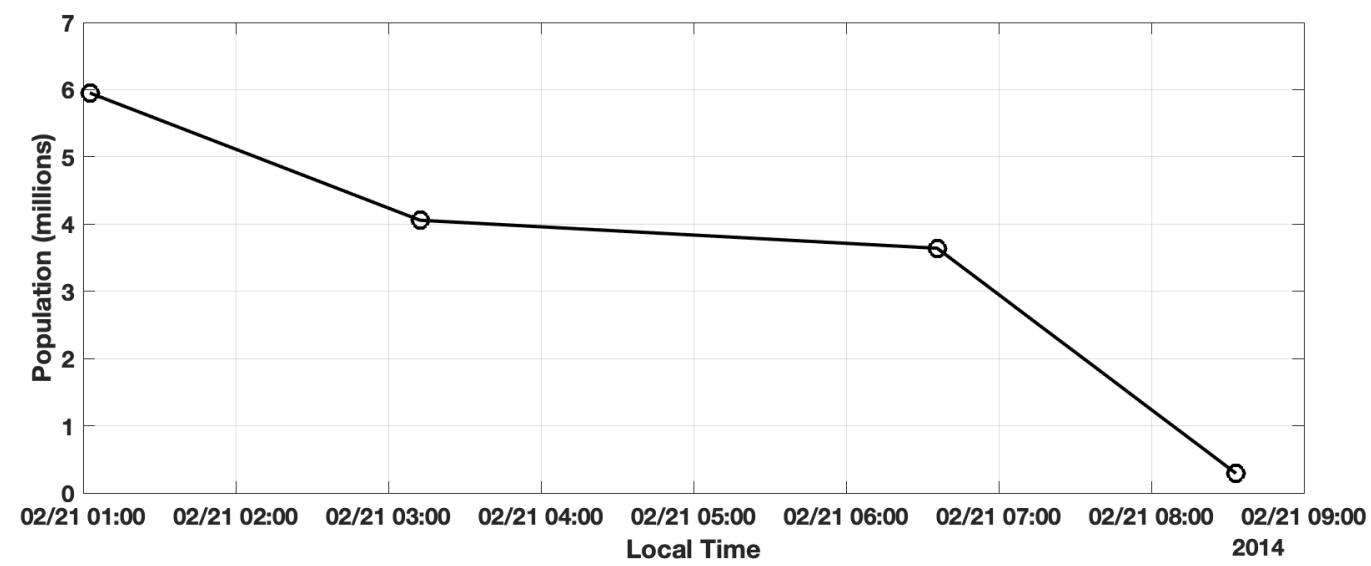

Figure 3-10: Herring population from instantaneous OAWRS imagery over entire survey region shown in Figure 3-9 for each instant as a function of time. Herring population is calculated by integrating OAWRS measurements of population density over regions where the population density is greater than $0.2 \mathrm{fish} / \mathrm{m}^{2}$, which is the critical population density at which large herring shoals were found to form [37, 38] (Appendix L.1).

where sensing is affected by attenuation from fish. OAWRS imagery of Norwegian springspawning herring is corrected for attenuation in a manner consistent with waveguide scattering theory in order to produce population density maps over more than one thousand square kilometers during dense shoaling activity. It is found that attenuation from dense herring groups forming after nautical sunset on February 20 resulted in reductions in sensing range of roughly $20 \%$. It has been previously shown that attenuation from Norwegian herring can be significant at the sensing frequencies and typical shoaling densities in this experiment, and attenuation can be significantly reduced in this environment by choosing

Figure 3-11 (facing page): Spatial herring distribution inhomogenieties seen in wide-area OAWRS imagery are undersampled in sparse line-transect surveys and lead to population estimates ranging from 0.5 to 2.5 times the total OAWRS population for the region shown. Assuming the spatial distribution of herring found in OAWRS population density maps and echosounder tracks from a 2001 survey in the same region (black line in A) [48], echosounder estimates of herring population are simulated by multiplying the average areal density along the echosounder transect within the survey region with the area of the survey region. The yellow contour in (A) denotes the OAWRS survey region for February 20, 17:55:49 (Figure 3-2), the blue contour in (A) denotes the OAWRS survey region for 21:13:19 (Figure 3-5), and the green box in (A) denotes the data collection site for February 21, 1:00-8:35 (Figure 3-9). Simulated echosounder population estimates as a percentage of OAWRS measurements are shown in (B), where yellow, blue, and green data correspond to simulations of echosounder estimates within the corresponding OAWRS sensing region. 

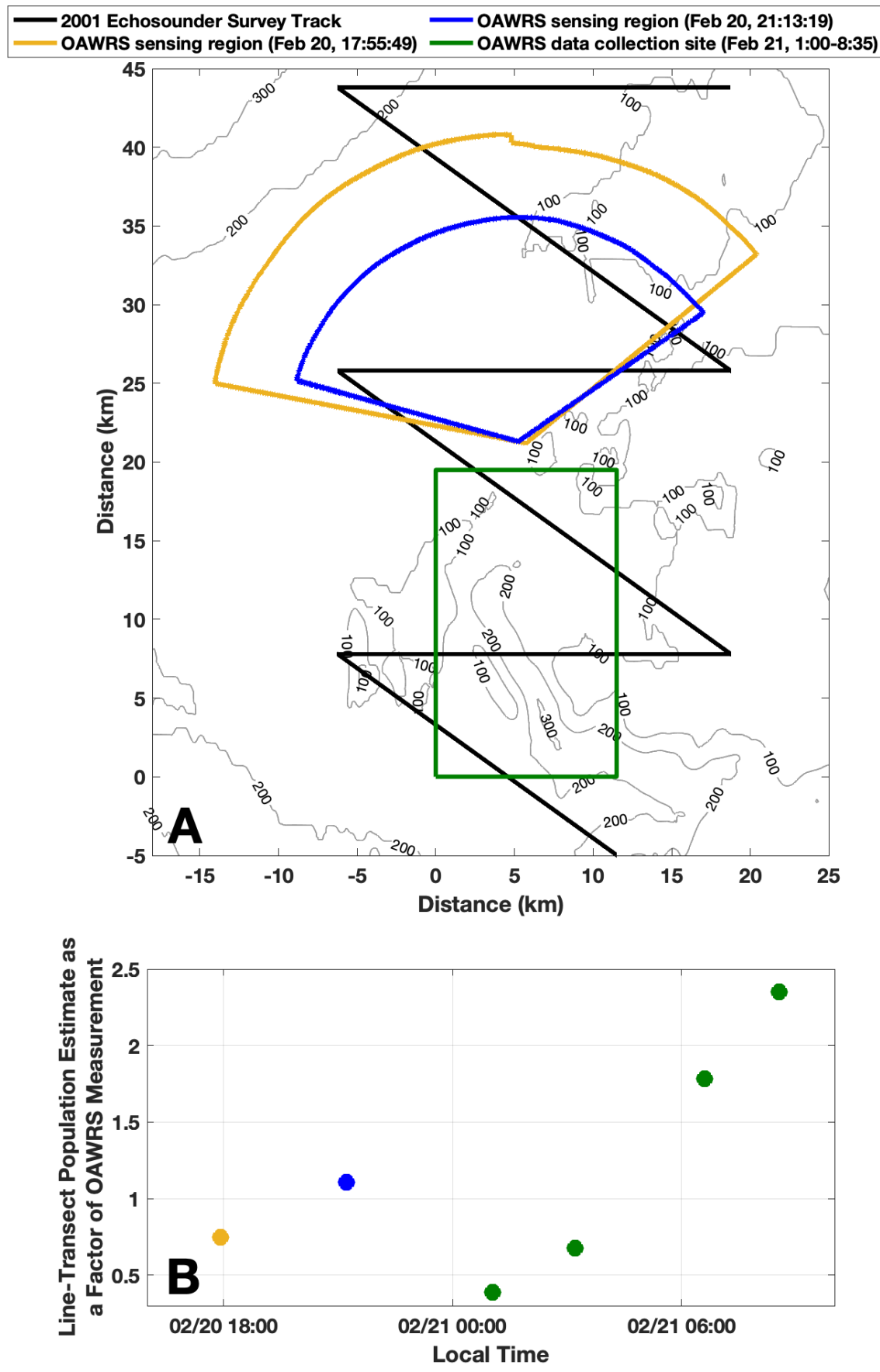
sensing frequencies above or below the swimbladder resonance of the herring $(>2 \mathrm{kHz}$ or $<500 \mathrm{~Hz}$ ) [15]. Such attenuation requires exceptionally high fish population densities over significant portions portions of the water column and extended ranges, and so was not observed previously in OAWRS fish sensing including Mid-Atlantic Bight herring [38], Gulf of Maine herring [26] and Lofoten cod [35].

\subsection{Conclusions}

Ocean Acoustic Waveguide Remote Sensing (OAWRS) is used to instantaneously monitor spawning Norwegian herring population densities over wide areas, spanning more than one thousand square kilometers, in the Nordic Seas near the Alesund trench where herring are known to spawn. Larger spawning shoals often spanning a few kilometers are instantaneously imaged in their entirety. Range reductions of roughly $20 \%$ in sensing range after sunset have been found due to the corresponding formation of larger and denser herring shoals after sunset for OAWRS transmissions near swimbladder resonance. This attenuation is corrected for in a manner consistent with waveguide scattering theory, leading to instantaneous wide-area OAWRS population density maps up to the sensing range of ambient noise limitation. Sensing range reductions due to attenuation from fish are shown to be theoretically predictable from waveguide scattering theory and observed population densities. Such sensing range reductions may also limit the ability of vocalizing marine mammals to echolocate in regions with dense fish shoals $[62,27]$. Spatial-temporal inhomogenieties in wide-area herring distributions observed by OAWRS indicate large errors are expected in population density estimates made from sparse line transect surveys through this region during spawning. This is due to the significant temporal and spatial aliasing of the non-homogeneous herring population density distributions observed by OAWRS. The population density maps provided by OAWRS can then be used in conjunction with conventional line-transect survey methods to efficiently provide more accurate estimates of herring populations and tempora-spatial distributions. 


\section{Chapter 4}

\section{The Effect of Attenuation from Fish}

on Passive Ocean Acoustic Waveguide Remote Sensing of Surface Vessels in

\section{the Ocean}

\subsection{Introduction}

Acoustics is the primary means of sensing self-radiating sources in the ocean such as manmade vehicles, geophysical phenomena, and vocalizing marine mammals [40, 53, 55]. Attenuation from fish, however, can reduce the intensity of acoustic signals and significantly decrease detection range for passive sensing in the ocean $[59,7]$. This makes it important to accurately predict attenuation from fish in long-range passive sensing. Attenuation from fish has been previously measured from fluctuations in the intensity of long-range acoustic signals caused by diel or seasonal shoaling patterns of the fish, including sardines in the Bristol Channel and groups of anchovies and sardines in the Gulf of Lion [59, 7, 12, 13]. In these experiments fish population densities were inferred from the reductions in signal intensity, since it is difficult to measure instantaneous population distributions across long ranges with conventional survey methods. More recently, wide area Ocean Acoustic Waveguide Remote Sensing (OAWRS) was used to observe fish shoals over thousands of square kilometers and simultaneously measure attenuation from these shoals within the active OAWRS transmis- 
sions [15]. Here the effect of attenuation from fish on passive ocean acoustic waveguide remote sensing (POAWRS) of ship-radiated tonals is investigated, where wide-area OAWRS imagery of herring groups in Norwegian spawning grounds is used in conjunction with in situ echosounder data to measure the size and population density of the fish groups occluding the propagation path.

POAWRS has been previously previously applied to detect, localize, and classify vocalizations from fin whales in the Norwegian Sea [18], baleen whale species and toothed whale species in the Gulf of Maine [23, 56, 21], and sperm whales along the US east coast [51] over continental shelf-scale regions. TemporalâĂŞspatial distributions of marine mammal vocalizations measured by POAWRS in the Gulf of Maine have been compared with herring population density distributions in order to provide insights into the predatorâĂŞprey dynamics in that ecosystem, and it was shown that marine mammals will spatially converge on fish spawning grounds and divide into separate foraging areas specific to each marine mammal species [55].

POAWRS has also been used to detect and characterize ship-radiated sound from surface vessels $[22,64,65]$. During previous OAWRS surveys of spawning herring in the Gulf of Maine and spawning capelin in Finnmark, Norway, ship-radiated tonals from research vessels were continuously measured with POAWRS over more than 10 hours and at ranges of up to $30 \mathrm{~km}$ [22]. During the OAWRS survey of Norwegian herring spawning grounds, POAWRS detections of ship-radiated tonals from a fishing vessel less than $20 \mathrm{~km}$ away were lost to ambient background noise for periods of more than 1.5 hours [22]. Here it is experimentally and theoretically shown that these detection losses are caused by attenuation from the herring shoals. The conditions determining whether attenuation from fish will significantly affect passive sensing are investigated here, and they are found to be similar to previouslydemonstrated conditions determining whether attenuation will affect active sensing [15]. Attenuation from herring shoals did not significantly affect ship-radiated tonals in the Gulf of Maine since the frequencies of the tonals were well below the resonance peak of the shoaling herring in the region. Attenuation from capelin shoals did not significantly affect tonals in the Finnmark region because the size and population density of the capelin shoals were not sufficiently high. No attenuation was observed in one-way acoustic transmissions during an OAWRS survey of herring in the Gulf of Maine [50], likely because the direct propagation path between the source and receiver did not cross the fish shoals and the 
transmission frequencies were below the swimbladder resonance peak of the shoaling fish [15].

Reductions in the intensity of ship-radiated tonals due to attenuation from fish are predicted using an analytical formualtion for acoustic propagation and scattering through an ocean waveguide with inhomogeneities [43]. This formulation has been previously shown to be consistent with experimental measurements of attenuation from fish to active OAWRS transmissions [15], as well as measurements of attenuation and temporal coherence loss in the presence of surface gravity waves, near-sea-surface air bubbles and internal waves $[4,5,20,8]$. Reductions in ship-radiated sound measured here are consistent with the predictions of the waveguide attenuation model, where the position, size, and population density of the fish groups occluding the propagation path are fully characterized using OAWRS imagery as well as in situ echosounder measurements. Common heuristic approaches that employ free space scattering assumptions for attenuation from fish are also investigated. While it has been theoretically shown that these heuristic approaches can be in disagreement with formulations that incorporate waveguide scattering and propagation effects (Chapter 2), here it is experimentally confirmed that the heuristic approaches can be in significant error for predicting attenuation in a waveguide environment.

It is experimentally shown that reductions in the intensity of ship-radiated tonals increase with the size and population density of fish groups occluding the propagation path, with reductions of more than $4 \mathrm{~dB}$ commonly observed for the Norwegian herring shoals studied here. These intensity reductions are shown to lead to prolonged detection losses as dense herring shoals continuously occlude the propagation path. Such intensity reductions and corresponding detection losses may be significant in other regions where dense fish groups congregate, and may present significant impediments to passive sensing of other acoustic sources such as vocalizing marine mammals and underwater vehicles.

\subsection{Materials and Methods}

\subsubsection{Measuring Reductions in Ship Tonal Intensity due to Fish-Attenuation}

Ship-radiated tonals from the FV Artus are detected by the POAWRS system over a threehour period during a survey of herring spawning grounds near Ålesund, Norway (Figure 4-1). Passive acoustic data is beamformed in the direction of the Artus using a large- 
aperture densely-sampled horizontal coherent receiver array towed behind the RV Knorr $[22,64,65]$, where the relative bearing of the Artus is determined using GPS data from both ships. Concurrent wide-area scattering strength maps generated by the monostatic OAWRS system are used to monitor herring groups within a $30 \mathrm{~km}$ radius of the Knorr (Appendix A). Active OAWRS transmissions do not interfere with the passive measurements shown here since the frequency of the Artus tonal studied here $(1584 \mathrm{~Hz})$ is well above the OAWRS sensing frequency $(955 \mathrm{~Hz})$.

Significant reductions in the received level of ship-radiated tonals from the Artus are observed when three herring shoals occlude the propagation path between 3:15-3:26 on February 21 (Figure 4-2). The decrease in sound pressure level of the Artus tonal due to attenuation from fish $\triangle S P L_{\text {measured }}$ is measured as the decrease in the received level beamformed in the direction of the Artus $(R L)$ corrected for transmission loss from spreading losses and seafloor scattering $(T L)$ after the fish groups occlude the propagation path, according to

$$
\Delta S P L_{\text {measured }}=(R L+T L)_{\mathrm{no} \mathrm{fish}}-(R L+T L)_{\mathrm{fish}}
$$

where $(R L+T L)_{\text {no fish }}$ corresponds to measurements where OAWRS imagery confirms that no significant fish groups occlude the propagation path from the Artus to the receiver (Figure $4-2 \mathrm{~A})$, and $(R L+T L)_{\text {fish }}$ corresponds to measurements where significant fish groups are observed in OAWRS imagery along the propagation path (Figure 4-2B). The received level $R L$ is calculated from the average received intensity at frequencies between $f_{c} \pm B / 2$, where $f_{c}=1584 \mathrm{~Hz}$ is the center frequency of the Artus tonal and $B=30 \mathrm{~Hz}$ is the $3 \mathrm{~dB}$-down bandwidth of the Artus tonal averaged over 30 seconds. Transmission loss from spreading losses and seafloor scattering $(T L)$ is defined as

$$
T L=10 \log _{10}\left((4 \pi)^{4}\left\langle\left|G\left(\boldsymbol{r} \mid \boldsymbol{r}_{\boldsymbol{S}} ; f, c\left(\boldsymbol{r}_{\boldsymbol{w}}\right), d\left(\boldsymbol{r}_{\boldsymbol{w}}\right)\right)\right|^{2}\right\rangle\right)
$$

where $G\left(\boldsymbol{r} \mid \boldsymbol{r}_{\boldsymbol{S}} ; f, c\left(\boldsymbol{r}_{\boldsymbol{w}}\right), d\left(\boldsymbol{r}_{\boldsymbol{w}}\right)\right)$ is the Green function between the source location $\boldsymbol{r}_{\boldsymbol{S}}=$ $\left(\boldsymbol{\rho}_{\boldsymbol{S}}, z_{S}\right)$ and the receiver location $\boldsymbol{r}$. A parabolic equation model [9] is used to calculate the Green functions in the range-dependent environment, where the conditional expectation over the sound speed is determined by averaging five Monte-Carlo realizations. Each MonteCarlo realization employs sound-speed profiles measured in the relevant environment (Figure F-1) every $500 \mathrm{~m}$ along the propagation path [1]. In the example shown in Figure 4-2 (3:15- 
3:26 on February 21$),(R L+T L)_{\text {no fish }}$ is equal to $164.6 \mathrm{~dB}$ with a standard deviation of $1.6 \mathrm{~dB}$, and $(R L+T L)_{\text {fish }}$ is equal to $160.7 \mathrm{~dB}$ with a standard deviation of $1.4 \mathrm{~dB}$. The decrease in the Artus tonal due to attenuation from fish $\triangle S P L_{\text {measured }}$ is then $3.9 \mathrm{~dB}$ with a standard deviation of $2.1 \mathrm{~dB}$.

\subsubsection{Predicting Reductions in Ship Tonal Intensity due to Fish-Attenuation}

Reductions in the intensity of ship-radiated tonals due to attenuation from fish are predicted using a normal-mode-based analytical theory for acoustic propagation and scattering through inhomogeneities in an ocean waveguide (Section 2.2). Predicted reductions depend on the population density distribution with respect to depth and range along the propagation path, where each shoal is characterized here assuming a constant areal population density $n_{A}$, mean shoal depth $z_{m}$, shoal vertical thickness $H$, mean range along the propagation path $\rho_{m}$ and horizontal width along the propagation path $W$. These variables are determined using a combination of OAWRS scattering strength maps and in situ echosounder measurements of the three shoals (Figure 4-3).

The population density $\left(n_{A}\right)$ and vertical distribution $\left(z_{m}, H\right)$ of the herring shoals occluding the propagation path are determined from in situ echosounder measurements of the specific shoals. Echosounder measurements of volumetric population density $n_{V}$ and areal population density $n_{A}$ are calculated according to Appendix L. The shoals are segmented by selecting regions where the areal population density is greater than the critical population density at which herring groups are known to form $\left(0.2 \mathrm{fish} / \mathrm{m}^{2}\right)[37,38]$, and the mean population density $n_{A}$ for each shoal is determined by averaging across these segments (Figure 4-3D-F). The mean depth $z_{m}$ and vertical thickness $H$ for each shoal are chosen so that the average volumetric population density with respect to depth $n_{V}(z)$ is greater than $10^{-3} \mathrm{fish} / \mathrm{m}^{3}$ at depths between $z_{m}-H / 2$ and $z_{m}+H / 2$ (Figure 4-3G-I).

The horizontal distribution of shoals along the propagation path $\left(\rho_{m}, W\right)$ is determined using OAWRS scattering strength measurements along the propagation path from the source (FV Artus) to the receiver (RV Knorr). OAWRS scattering strength is typically measured by correcting transmissions for source level, areal resolution footprint, spreading loss and seafloor attenuation $[37,38,26,19]$, however the OAWRS transmissions in this environment have been previously shown to be affected by attenuation from herring shoals [15], so these measurements are referred to as "fish-attenuated scattering strength" (Appendix A). The 
mean range of each shoal along the propagation path $\rho_{m}$ and horizontal width of each shoal along the propagation path $W$ are chosen so that the fish-attenuated scattering strength is $5.6 \mathrm{~dB}$ greater than background seafloor scattering at ranges between $\rho_{m}-W / 2$ and $\rho_{m}+W / 2$ (Figure 4-3J), where $5.6 \mathrm{~dB}$ is the standard deviation of an acoustic measurement after saturated multipath propagation when the time-bandwidth product is one $[16,34]$. Measured values of $n_{A}, z_{m}, H, \rho_{m}$, and $W$ for the three herring shoals studied here are shown in Table 4.1.

\subsection{Results}

The predictions of the waveguide attenuation model are found to be in agreement with measured reductions in the Artus tonal due to attenuation from herring, where the size, position, and population density of the three shoals occluding the propagation path are fully characterized by OAWRS imagery as well as in situ echosounder measurements (Figure 4-4). The reduction in sound pressure level $\triangle S P L$ is modeled according to Equation 2.10, where the volumetric population density $n_{V}$ is expressed with respect to range $\rho$ and depth $z$ assuming discrete shoals with constant population densities, according to:

$n_{V}(\rho, z)= \begin{cases}\left(n_{A, k} / H_{k}\right) & \rho_{m, k}-W_{k} / 2<\rho<\rho_{m, k}+W_{k} / 2 \& z_{m, k}-H_{k} / 2<z<z_{m, k}+H_{k} / 2 \\ 0 & \text { otherwise }\end{cases}$

where $n_{A, k}$ is the areal population density of shoal $k, z_{m, k}$ is the mean depth of shoal $k, H_{k}$ is the vertical thickness of shoal $k, \rho_{m, k}$ is the mean range of shoal $k$ along the propagation path and $W_{k}$ is the horizontal width of shoal $k$ along the propagation path (Table 4.1). In the upward-refracting underwater environment studied here, dominant lower order acoustic modes are concentrated in the upper water column. Since the observed fish groups are concentrated in the lower water column, these lower order modes will experience almost no attenuation from the fish groups (Figure 4-4). Modeled $\triangle S P L$ is found to be in agreement with measurements at the receiver depth used in this experiment. Free-space-like factored formulations that ignore these depth-dependent modal attenuation effects (Equation 14 of [15]) overestimate the loss due to attenuation from fish by more than $5 \mathrm{~dB}$ (Figure 4-4F).

Reductions in the intensity of the Artus tonal and corresponding detection losses are 


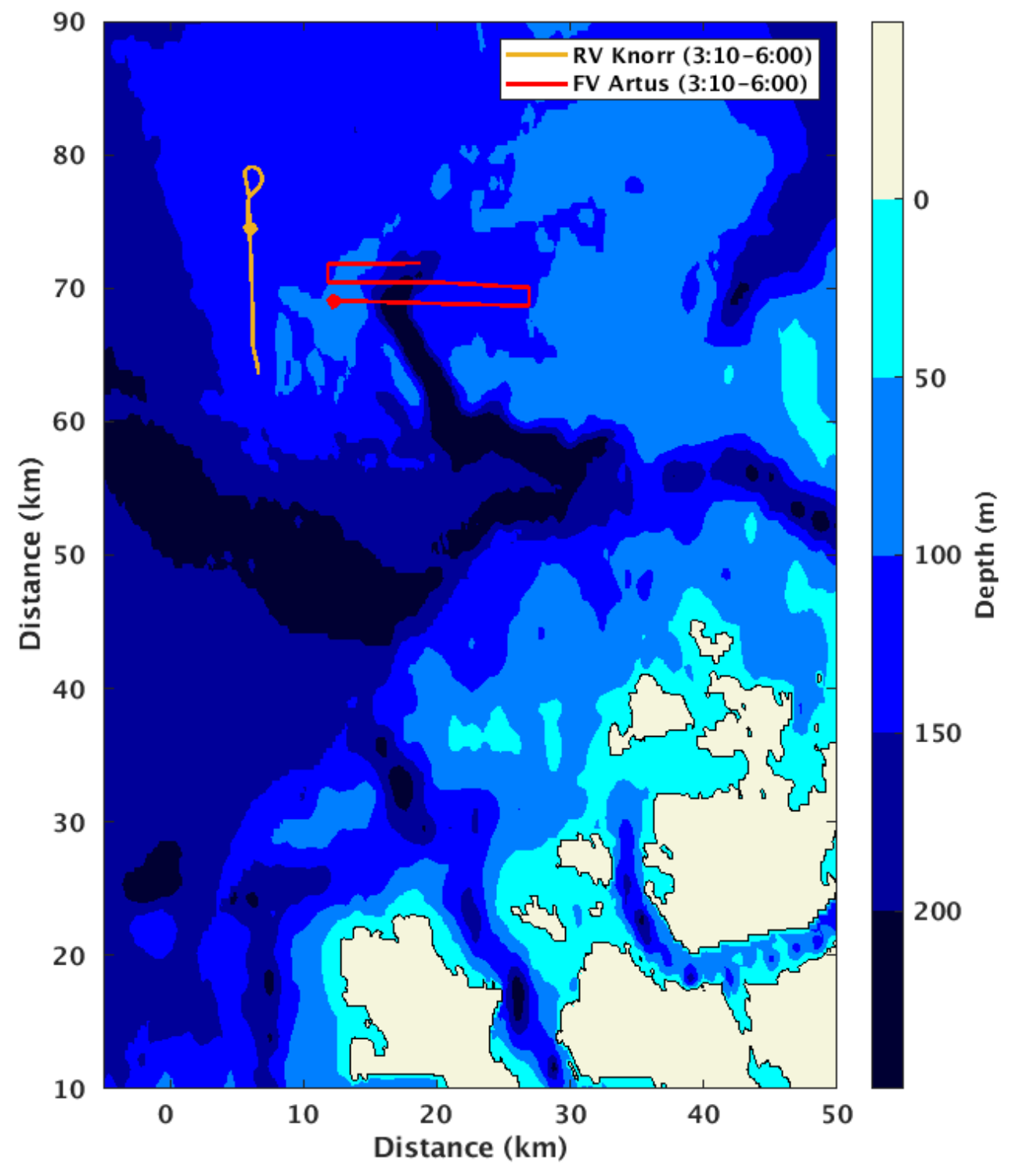

Figure 4-1: Ship-radiated tonals from the FV Artus (red) are intermittently detected by a horizontal coherent receiver array towed by the RV Knorr (orange) in herring spawning grounds near Ålesund, Norway between 3:10 and 6:00 UTC on February 21, 2014. Red and orange dots denote the starting position of the tracks at 3:10 UTC. 

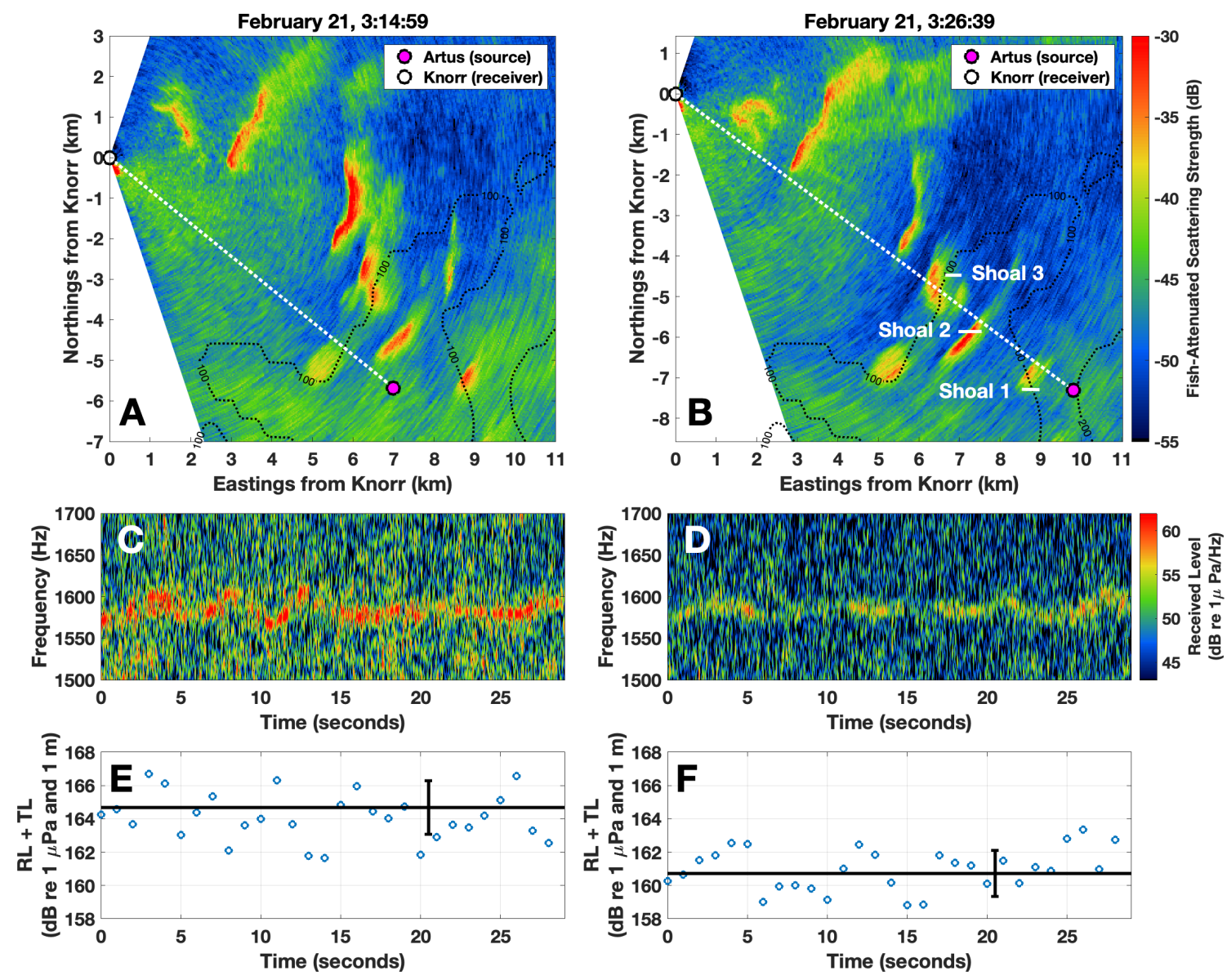

Figure 4-2: Significant reductions in the intensity of a ship-radiated tonal from the FV Artus are observed when a group of three herring shoals occlude the propagation path. At 3:14:59, no fish groups are observed in wide-area OAWRS imagery along the propagation path from the Artus to the receiver array (A). At 3:26:39, OAWRS imagery reveals three fish shoals occluding the propagation path (B). Spectrograms of the $1584 \mathrm{~Hz}$ tonal from the Artus beamformed in the direction of the Artus are shown in (C) and (D), where significant reductions in the received level are observed after the fish groups occlude the propagation path. The received level within the bandwidth of the Artus tonal $(R L)$ corrected for transmission loss from spreading and seafloor attenuation $(T L)$ is shown in $(\mathrm{E})$ and $(\mathrm{F})$. Blue dots denote $R L+T L$ measurements averaged over $1 \mathrm{~s}$ intervals within the frequency band of the Artus tonal (1569-1599 Hz), and black lines denote the mean and standard deviation of these $1 \mathrm{~s}$ measurements. The three white lines in (B) correspond to echosounder transects of the three herring shoals shown in Figure 4-3. Fluctuations in the measured scattering strength of herring shoals between (A) and (B) are caused by attenuation from fish to OAWRS signals, which has been previously investigated in [15]. Reductions in the received level between $1500-1700 \mathrm{~Hz}(\mathrm{C}$ and $\mathrm{D})$ are likely caused by attenuation from herring of broadband noise from the Artus. Black dotted lines in (A) and (B) designate bathymetric contours. 
shown to be strongly correlated with dense herring groups occluding the propagation path. The population density of herring groups between the source and receiver is calculated at each time interval by interpolating an OAWRS population density map (Figure 4-5) between the known locations of the Artus and the Knorr. During a period between 3:28 and 5:07, the tonal from the Artus is not detected by the POAWRS system. This loss of detection is shown to be correlated with herring groups occluding the propagation path with horizontal widths greater than $1 \mathrm{~km}$ and population densities above $0.2 \mathrm{fish} / \mathrm{m}^{2}$, which is the critical population density at which large herring shoals were found to form [37, 38] (Figure 4-6).

Reductions in the Artus tonal due to attenuation from fish are predicted between 3:10 and 6:00 from population density measurements using Equation 2.10. In the absence of individual echosounder measurements of herring depth for each shoal, attenuation is modeled assuming the same depth distribution for all shoals. The volumetric population density $n_{V}$ with respect to range $\rho$ and depth $z$ is then expressed as:

$$
n_{V}(\rho, z)=n_{A}(\rho) p(z)
$$

where $n_{A}(\rho)$ is the areal population density measured by OAWRS (Figure 4-5) and $p(z)$ is the probability distribution function of herring depth, which is determined from echosounder measurements during the experiment and explicitly defined in Appendix L. Predicted reductions in the intensity of the Artus tonal are shown to be consistent with measured reductions

Figure 4-3 (facing page): In order to predict reductions in ship-radiated tonals from the FV Artus due to attenuation from herring, the areal population density, vertical position, and horizontal position of the three herring shoals labeled in Figure 4-2B are characterized using a combination of in situ echosounder measurements and OAWRS imagery. The areal population density and vertical distribution are estimated from echosounder measurements of the shoals recorded less than two hours from the OAWRS transmission (A-C). The areal density along each echosounder transect is shown in (D-F). The three shoals are segmented by choosing regions where the measured areal population density is above the critical population density at which herring groups were found to form (0.2 fish $/ \mathrm{m}^{2}$, black solid lines in D-F) [37, 38]. The vertical position of these shoals is determined by selecting depths where the average volumetric population density along each segment is greater than $10^{-3} \mathrm{fish} / \mathrm{m}^{2}$ (black solid lines in G-I). The horizontal position of the shoals along the propagation path from the FV Artus to the receiver is estimated from OAWRS measurements of fish-attenuated scattering strength along the propagation path $(\mathrm{J})$. The horizontal position of the shoals (gray boxes in $\mathrm{J}$ ) is determined by selecting regions where the fish-attenuated scattering strength is $5.6 \mathrm{~dB}$ greater than the mean seafloor scattering level measured in this region $(-50 \mathrm{~dB})$. 

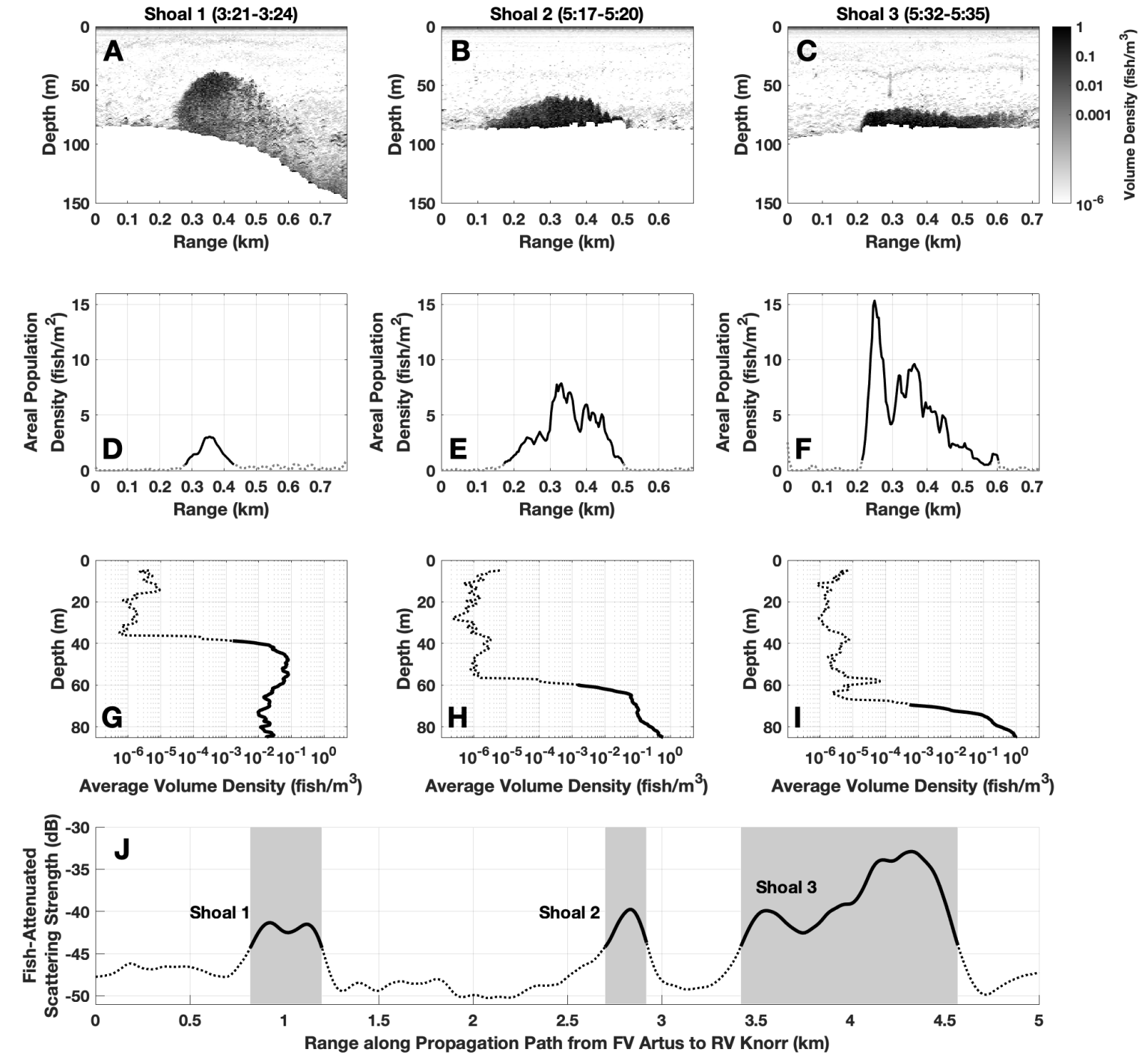

Average Volume Density (fish/ $\mathrm{m}^{3}$ ) 
and corresponding detection losses (Figure 4-7). Variations in ship speed are less than 0.5 $\mathrm{m} / \mathrm{s}$ during this time period, indicating that changes in the source level of the Artus tonal over time are not significant enough to lead to the detection losses observed here (Figure O-1).

Reductions in the Artus tonal due to attenuation from fish are predicted between 3:10 and 6:00 from population density measurements using Equation 2.10 (Figure 4-5). In the absence of individual echosounder measurements of herring depth for each shoal, attenuation is modeled assuming the same depth distribution for all shoals based on an aggregation of echosounder measurements during this three-hour period (Figure L-4). Predicted reductions in the intensity of the Artus tonal are shown to be consistent with measured reductions and corresponding detection losses (Figure 4-7). Variations in ship speed are less than $0.5 \mathrm{~m} / \mathrm{s}$ during this time period, indicating that changes in the source level of the Artus tonal over time are not significant enough to lead to the detection losses observed here (Figure O-1).

While attenuation from herring in Ålesund waters led to prolonged detection losses for tonals from the FV Artus, attenuation from fish did not affect POAWRS detections of ship-radiated tonals during previous surveys of herring in the Gulf of Maine and Norwegian capelin off the coast of Finnmark [22]. Reductions in the intensity of ship-radiated tonals due to attenuation from fish is predicted in all three environments, and the presence or absence

Figure 4-4 (facing page): The predictions of the waveguide attenuation model are consistent with measured reductions in the Artus tonal due to attenuation from herring. The population density distributions of the three shoals measured in Figure 43 as well as the position of the source and receiver are shown in (A). A source depth of $6 \mathrm{~m}$ (magenta dot in A) is chosen to correspond with the draft depth of the FV Artus [22], and the depth of the receiver array towed by the RV Knorr is $50 \mathrm{~m}$ (white dot in A). Attenuation coefficients associated with given modes and their coupling depend on the specific modal contributions at the depths where the fish groups are present (B-D). Since the Alesund underwater environment is upward-refracting (Figure F-1), the lowest order modes concentrated in the upper water column will experience almost no attenuation from the fish groups which are concentrated in the bottom half of the water column (E). As a result, the predicted reduction in sound pressure level due to attenuation from fish $(\triangle S P L)$ is approximately $10 \mathrm{~dB}$ lower when the receiver is in the upper water column compared to the lower water column (F). The modeled decrease in the received level (blue solid line in F) is within a standard deviation of measurements at the relevant receiver depth (red data in F). The loss due to attenuation estimated by the free-space-like factored approximation is not sensitive to changes in receiver depth, and predictions are more than $5 \mathrm{~dB}$ greater than measurements (blue dotted line in F). Water density is modeled as $1000 \mathrm{~kg} / \mathrm{m}^{2}$, seafloor density as $1900 \mathrm{~kg} / \mathrm{m}^{2}$ and seafloor sound speed as $1700 \mathrm{~m} / \mathrm{s}$. 

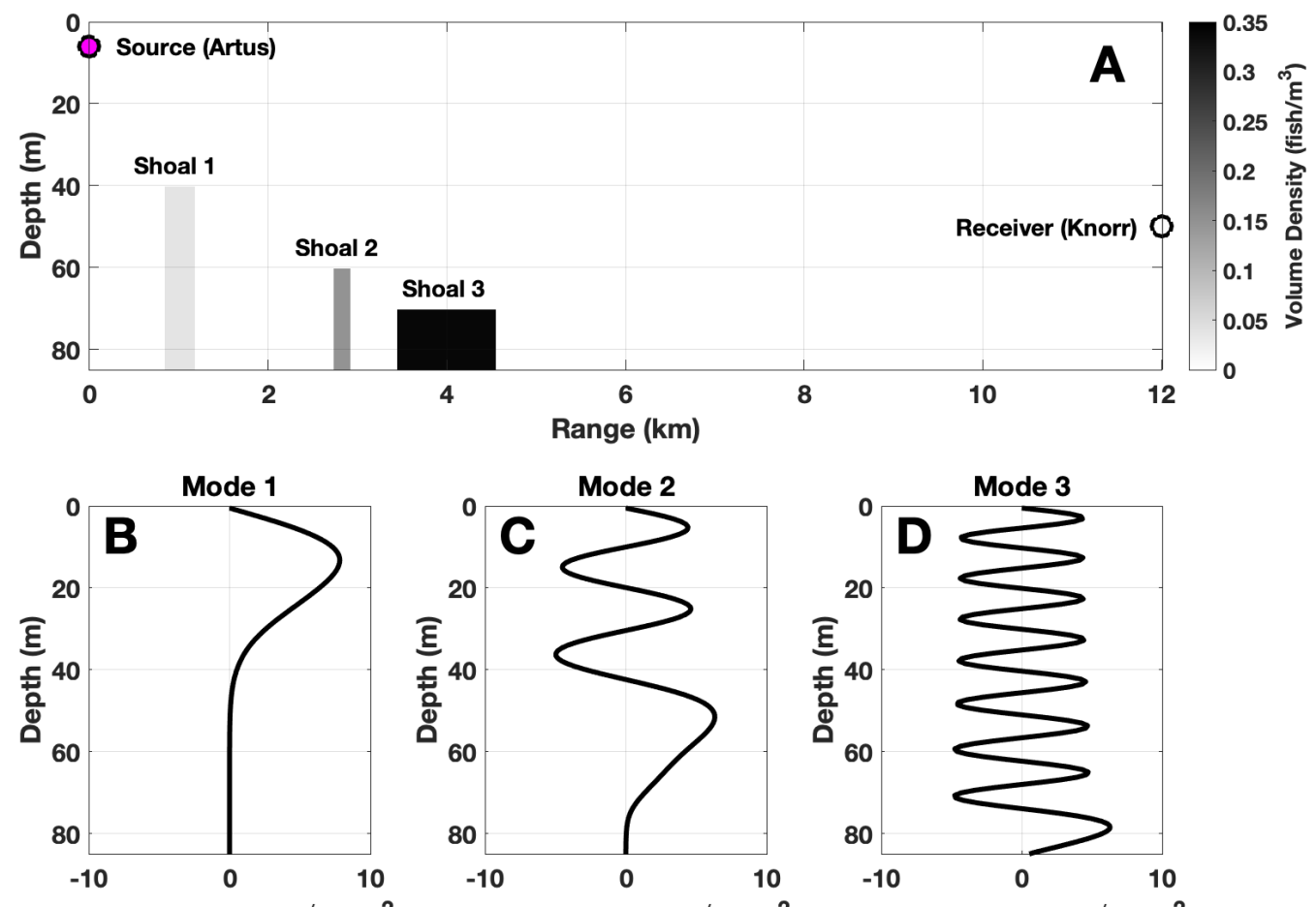

Modal Amplitude $\left(\sqrt{ } \mathrm{kg} / \mathrm{m}^{2}\right)$

Modal Amplitude $\left(\sqrt{ } \mathrm{kg} / \mathrm{m}^{2}\right)$

Modal Amplitude $\left(\sqrt{ } \mathrm{kg} / \mathrm{m}^{2}\right)$
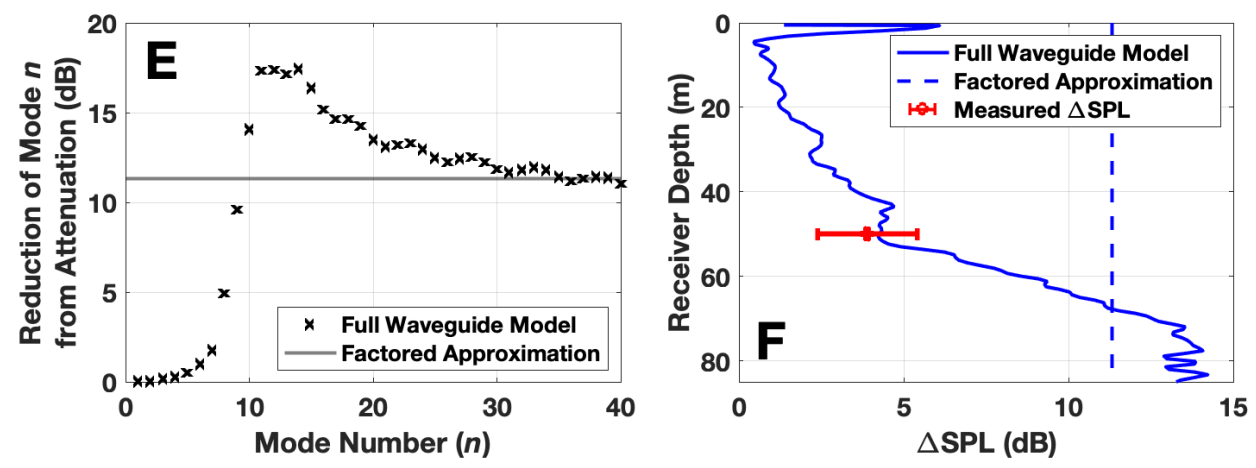
Table 4.1: Parameters Characterizing the three Herring Shoals in Figure 4-3

$\begin{array}{lccccc}\text { Areal } & \begin{array}{c}\text { Shoal } \\ \text { Density } \\ \left(\mathbf{f i s h} / \mathbf{m}^{\mathbf{2}}\right)\end{array} & \begin{array}{c}\text { Septh } \\ (\mathbf{m})\end{array} & \begin{array}{c}\text { Shoal Vertical } \\ \text { Thickness } \\ (\mathbf{m})\end{array} & \begin{array}{c}\text { Distance Along } \\ \text { Propagation Path }\end{array} & \begin{array}{c}\text { Shoal Horizontal } \\ \text { Thickness } \\ (\mathbf{k m})\end{array} \\ \text { Shoal 1 } & 1.7 & 62.5 & 45 & 1.01 & 0.31 \\ \text { Shoal 2 } & 3.7 & 72.5 & 25 & 2.82 & 0.17 \\ \text { Shoal 3 } & 5.1 & 77.5 & 15 & 4.00 & 1.10\end{array}$

of significant attenuation is found to be consistent with theoretical predictions. Attenuation is negligible for tonals from the RV Delaware II in the Gulf of Maine since the frequencies of the tonals were well below the resonance peak of the spawning herring in that environment (Figure 4-8A). Predicted losses due to attenuation from Finnmark capelin are less than $1 \mathrm{~dB}$ because the horizontal width of observed capelin shoals is not large enough to lead to significant attenuation $(<0.25 \mathrm{~km}$, Figure 4-8B). Significant attenuation from Ålesund herring is predicted at the frequency of the tonal from the FV Artus, which is consistent with observed intensity reductions during the survey (Figure 4-8C). This analysis demonstrates that the waveguide attenuation formulation can be used to determine the conditions for significant attenuation from fish for long-range passive sensing in ocean waveguide environments.

\subsection{Discussion}

Measured reductions in ship-radiated tonals due to attenuation from fish are shown to be in agreement with a normal-mode based formulation for acoustic propagation through inhomogeneities in a waveguide. Common heuristic approaches that employ free space scattering assumptions disagree with measurements by more than $5 \mathrm{~dB}$. While the potential for such heuristic approaches to incorrectly predict attenuation from fish in a waveguide environment has been previously discussed in theory [15], to our knowledge the results shown here are the first experimental evidence that attenuation formulations ignoring waveguide physics can be in significant error. The conditions determining whether attenuation from fish will significantly affect passive sensing are investigated, and they are found to be similar to previouslydemonstrated conditions for active sensing in the ocean [15]. These results demonstrate that the waveguide attenuation formulation can be used to determine the conditions for significant attenuation from fish for long-range passive sensing. 


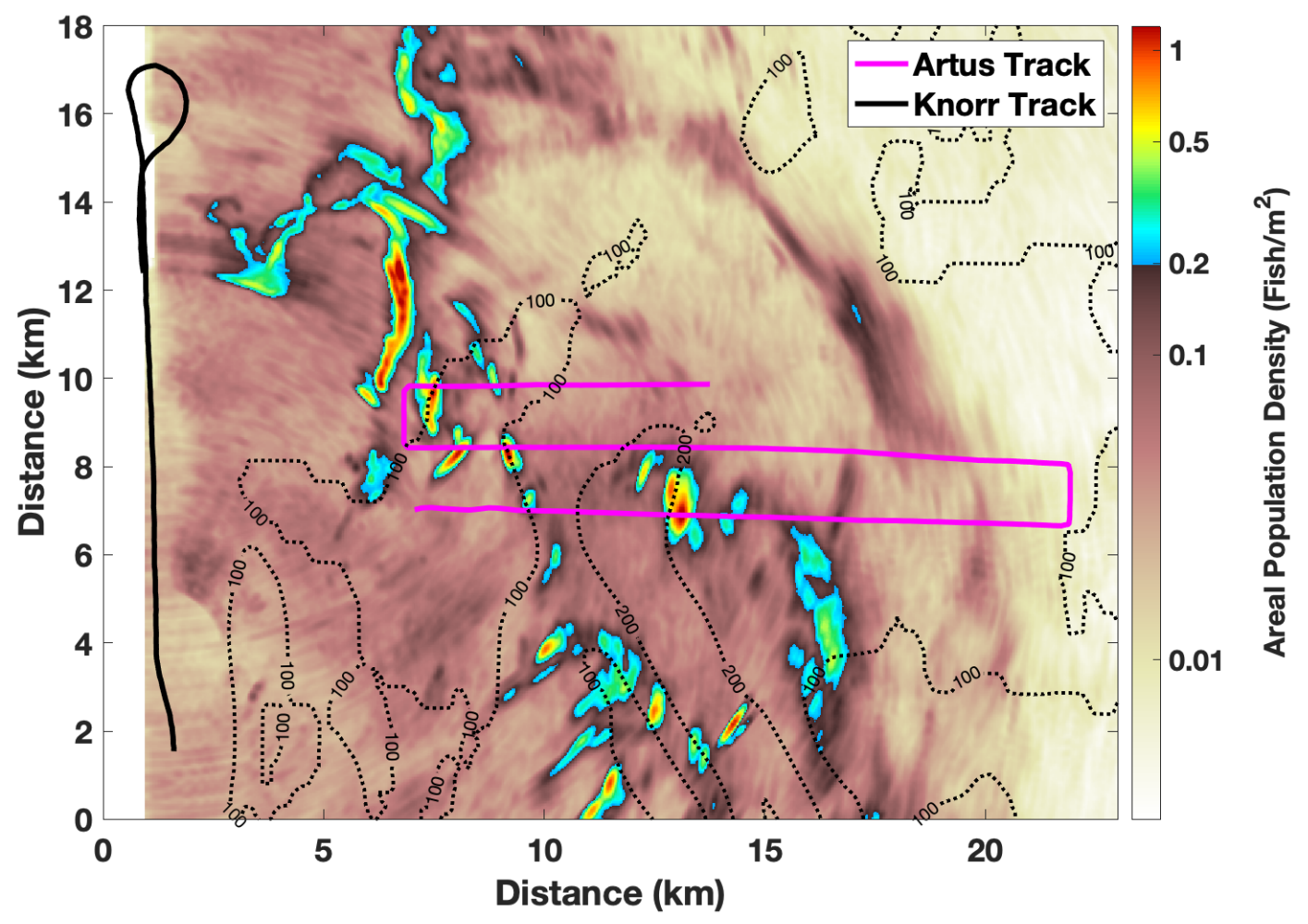

Figure 4-5: OAWRS map of herring areal population density between 3:00 and 6:00. Since attenuation from fish prevented the entire region from between instantaneously surveyed, the areal population density map is generated from five instantaneous OAWRS transmissions between 3:00 and 6:00 on February 21, 2014 (3:04:09, 3:24:09, 4:19:09, 5:04:09, and 5:39:59) according to Appendix A. Reductions in the received level of the Artus tonal between 3:10 and 6:00 shown in Figure 4-7 are calculated using the population density between the Artus (pink line) and the Knorr (black line). The color scale is chosen so that the transition from brown to blue occurs at $0.2 \mathrm{fish} / \mathrm{m}^{2}$, which is the critical population density at which large herring shoals were found to form $[37,38]$. Black dotted lines denote bathymetric contours. 

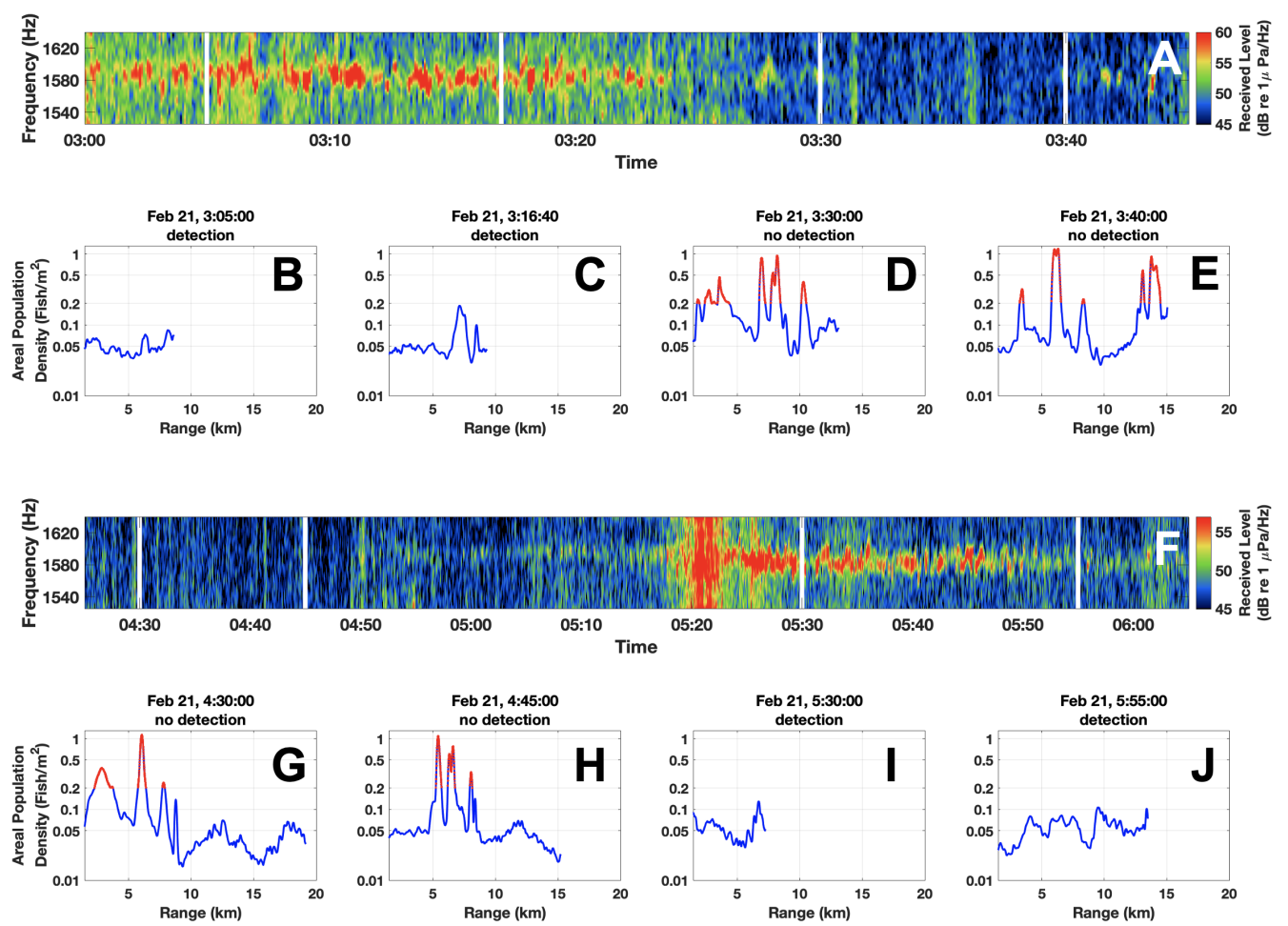

Figure 4-6: Detections of ship-radiated tonals from the FV Artus are lost to ambient noise when dense herring shoals occlude the propagation path. Spectrograms of the received level beamformed in the direction of the Artus are shown in (A) and (F). At approximately 3:28 on February 21, the signal from the Artus falls below ambient background noise (A). Before this loss of detection, OAWRS measurements of herring population density between the source and receiver are mostly below $0.2 \mathrm{fish} / \mathrm{m}^{2}$, which is the critical population density at which large herring shoals were found to form (B,C) [37, 38]. After the loss of detection, multiple fish groups are observed along the propagation path with population densities above $0.2 \mathrm{fish} / \mathrm{m}^{2}$ (red data in $\mathrm{D}, \mathrm{E}$ ). The signal from the Artus is detected again at approximately 5:10 (F). Before detection at 5:10 there are still dense fish groups occluding the propagation path with population densities above $0.2 \mathrm{fish} / \mathrm{m}^{2}$ (red data in $\mathrm{G}, \mathrm{H})$. After the Artus is detected, the population densities are below $0.2 \mathrm{fish} / \mathrm{m}^{2}$ $(\mathrm{I}, \mathrm{J})$. Measurements of herring areal population density shown here are from the OAWRS population density map shown in Figure 4-5. Vertical white lines in (A) and (F) correspond to times when measurements in (B-E) and (G-J) are respectively made. Broadband noise from the Artus is also observed between 3:00 and 3:28 in (A), and the strong broadband signal between 5:17 and 5:35 in (F) is likely caused by propeller cavitation from the Artus $[64,65]$. 


\subsection{Conclusions}

It is experimentally and theoretically shown that attenuation from dense herring groups in Norwegian spawning grounds can reduce the intensity of ship-radiated tonals and lead to prolonged detection losses for passive sensing of surface vessels in an ocean waveguide. Such detection losses can create significant impediments to passive acoustic sensing of underwater vehicles, marine life, and geophysical phenomena. Here, wide-area OAWRS imagery is used in conjunction with echosounder data to measure the size, position, and population density of every major fish shoal occluding the propagation path from source to receiver. Reductions in signal intensity due to attenuation from fish are predicted using an analytical theory for acoustic propagation through inhomogeneities in an ocean waveguide, and measured intensity reductions are found to be in agreement with theoretical predictions. Common heuristic approaches that employ free space scattering assumptions for attenuation from fish groups are found to be in disagreement with measurements by more than $5 \mathrm{~dB}$. To our knowledge, this the first experimental evidence that free-space-like factored approximations can be in error for predicting attenuation from fish in an ocean waveguide.

Figure 4-7 (facing page): Reductions in the received level of the Artus tonal between 3:10 and 6:00 on February 21 and corresponding detection losses are consistent with predicted reductions due to attenuation from fish. The received level beamformed in the direction of the Artus within the frequency band of the Artus tonal (1569-1599 Hz) is shown in (A). Red data indicates times when the Artus tonal was detected above background noise, while black data indicates times then the Artus tonal was not detected (Appendix N). The received level corrected for transmission loss from spreading losses and seafloor attenuation $(R L+T L)$ is shown in $(\mathrm{B})$ for data where the Artus tonal is detected above ambient noise. The reduction in received level due attenuation from fish $(\triangle S P L)$ is predicted in (C) using the OAWRS map of herring population density shown in Figure 4-5. The measured $4 \mathrm{~dB}$ reduction in $R L+T L$ between 3:15 and 3:26 (B) is consistent with the predicted loss due attenuation from fish during this time $(\mathrm{C})$. After the detection loss at 3:28, the signal remains undetected until the predicted loss due to attenuation $(\triangle S P L)$ falls below $2 \mathrm{~dB}$ after 5:00. The gap in the data between 3:38 and 4:15 is from a period where both the RV Knorr and the FV Artus are turning. The gap in the data between 5:15 and 5:20 is from a period where the Artus is turning. The approximately 4 $\mathrm{dB}$ increase in $R L+T L$ after this gap in (B) is likely caused by this change in orientation, since the Artus turns away from the Knorr and creates a more direct path from the Artus propellor to the receiver. 

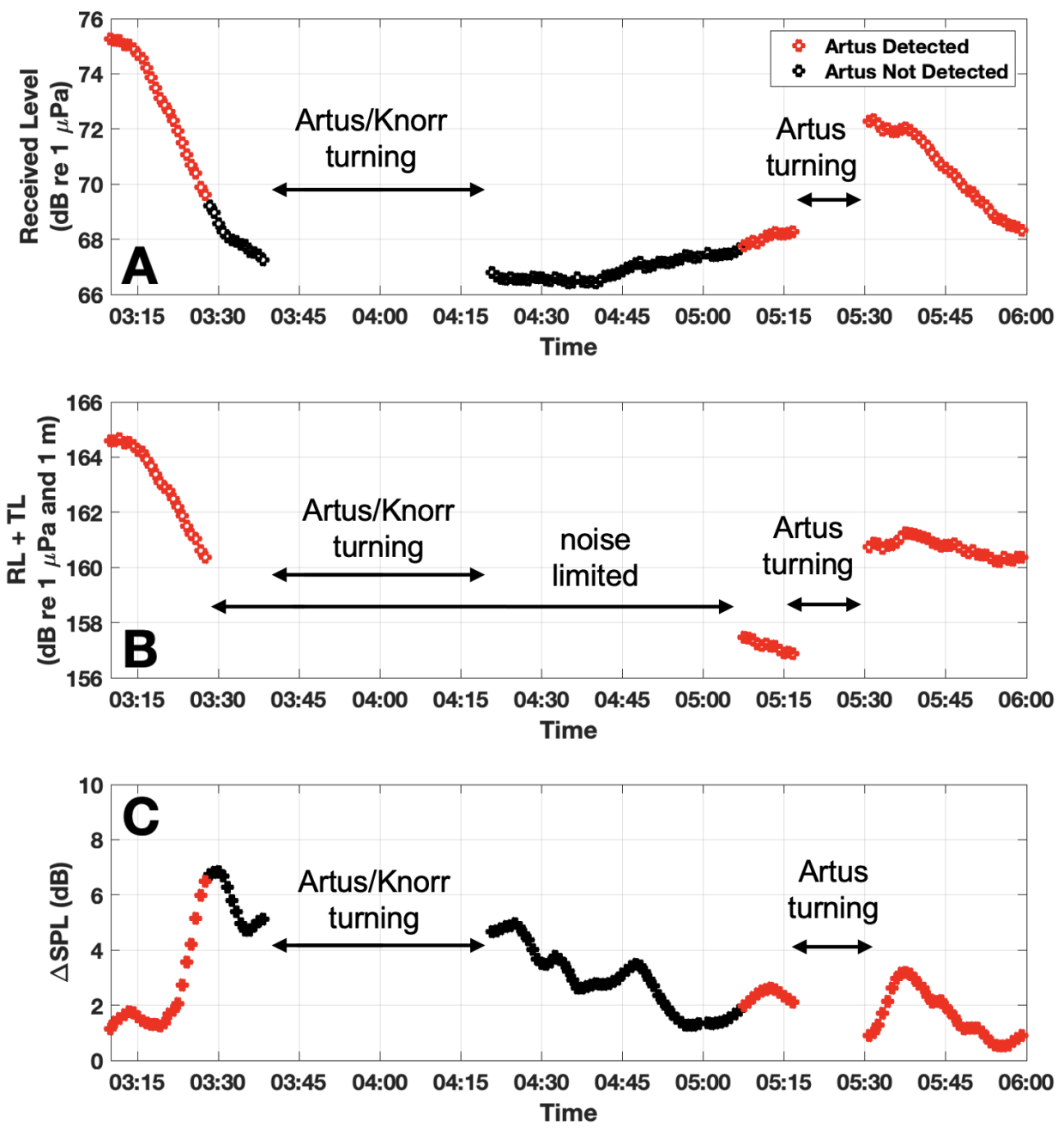

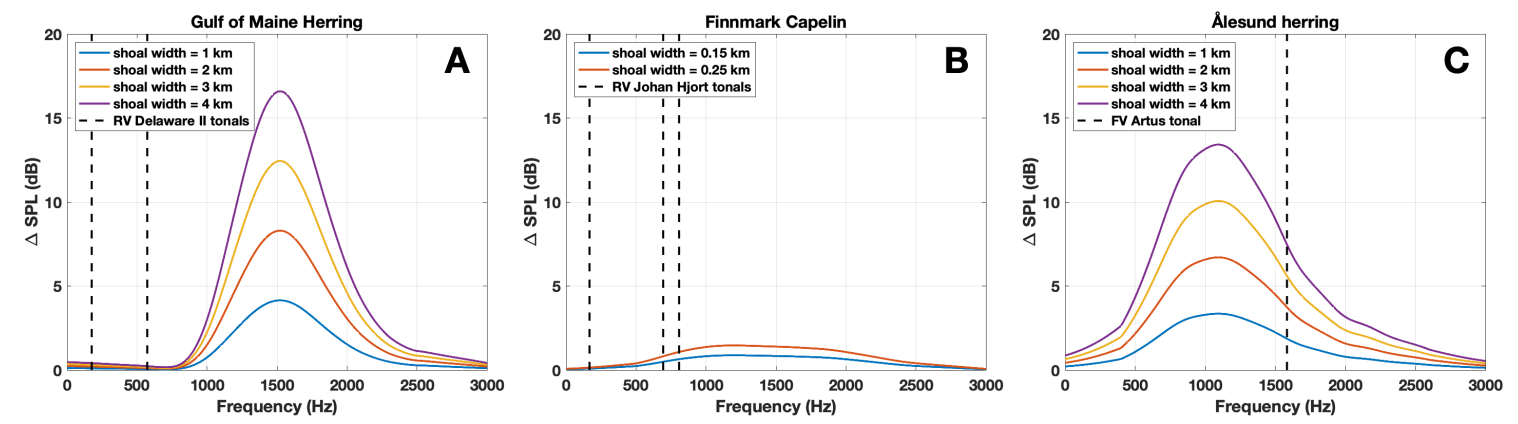

Figure 4-8: The decrease in the intensity of ship-radiated tonals due to attenuation from fish $(\triangle S P L)$ is predicted here for previous POAWRS surveys in three continental shelf environments: herring in the Gulf of Maine, capelin in Finnmark waters, and herring in Ålesund waters. The magnitude of $\triangle S P L$ depends on the width of the shoals along the propagation path between the source and receiver. Tonals from the RV Delaware II (black dotted lines in A) are well below the resonance peak of the herring in the Gulf of Maine and predicted losses due to attenuation are less than $0.3 \mathrm{~dB}$. The width of the observed capelin shoals in Finnmark are less than $0.2 \mathrm{~km}$, and corresponding losses due to attenuation are predicted to be less than $1 \mathrm{~dB}$ at the frequencies of tonals from the RV Johan Hjort (black dotted lines in B). Predicted attenuation from herring in Alesund is significant at the frequency of the tonal from the FV Artus (black dotted line in C). Physical parameters used for modeling attenuation in each environment are shown in Table 2.1, and measured sound speed profiles in each environment are shown in Figure F-1. 
Table 4.2: Environmental parameters used for attenuation calculations in Figure 4-8

\begin{tabular}{|c|c|c|c|c|c|c|}
\hline & Areal & Water & Shoal & Shoal Vertical & Neutral & Mean Fish \\
\hline Environment/Species & $\begin{array}{l}\text { Density } \\
\left(\text { fish } / \mathbf{m}^{2}\right)\end{array}$ & $\begin{array}{l}\text { Depth } \\
(\mathbf{m})\end{array}$ & $\begin{array}{l}\text { Depth } \\
(\mathbf{m})\end{array}$ & $\begin{array}{c}\text { Thickness } \\
\text { (m) }\end{array}$ & $\begin{array}{c}\text { Buoyancy Depth } \\
(\mathbf{m})\end{array}$ & $\begin{array}{l}\text { Length } \\
(\mathrm{cm})\end{array}$ \\
\hline Gulf of Maine herring & $2^{\mathrm{a}}$ & $200^{\mathrm{a}}$ & $150^{\mathrm{a}}$ & $30^{\mathrm{a}}$ & $82^{\mathrm{a}}$ & $24^{\mathrm{a}}$ \\
\hline Finnmark capelin & $10^{\mathrm{b}}$ & $300^{\mathrm{b}}$ & $30^{\mathrm{b}}$ & $40^{\mathrm{b}}$ & $10^{\mathrm{c}}$ & $17^{\mathrm{d}}$ \\
\hline Ålesund herring & $2^{\mathrm{e}}$ & $120^{\mathrm{e}}$ & $80^{\mathrm{d}}$ & $80^{\mathrm{d}}$ & $0^{\mathrm{e}}$ & $34^{\mathrm{d}}$ \\
\hline
\end{tabular}

${ }^{\mathrm{a}}[19],{ }^{\mathrm{b}}$ Measured from echogram data collected during the experiment, ${ }^{\mathrm{c}}[26],{ }^{\mathrm{d}}$ Measured from trawl samples collected during the experiment, ${ }^{\mathrm{e}}[15]$. 


\section{Appendix A}

\section{Measurement of Scattering Strength in OAWRS Images}

Data presented here is from an OAWRS experiment conducted in 2014 to survey fish populations in the Nordic Seas via continuous monitoring with instantaneous wide-area sensing. Roughly 10,000 active transmissions were recorded at frequencies between 850 and $1600 \mathrm{~Hz}$. The experiment covered multiple species in four regions in the Nordic Seas: herring in the Ålesund region were studied from 18-21 February, cod in the Lofoten region were studied on

23 February and 5-7 March, capelin in the Finnmark region were studied from 26-28 February and 1-3 March, and capelin in the Tromso region were studied from 28 February through 1 March. OAWRS data was produced from active transmissions of $1 \mathrm{~s}$ duration linearfrequency-modulated waveforms from a vertical source array attached to the research vessel (Figure A-1). Scattered returns from environmental features are received by a horizontal line array towed by the same research vessel with multiple nested sub-apertures. Three linear apertures of the receiver array, i.e., the low-frequency (LF) aperture, the mid-frequency (MF), and the high frequency (HF) aperture, consist of 64 equally spaced hydrophones with respective inter-element spacing of $1.5 \mathrm{~m}, 0.75 \mathrm{~m}$, and $0.375 \mathrm{~m}$. Images are generated by beamforming, matched filtering, and charting scattered returns, using nonuniformly-spaced combinations of the LF, MF, and HF apertures, as described in [57].

In cases where there is not significant attenuation from fish, scattering strength $S S$ can be calculated [37, 38, 26, 19] from

$$
S S=10 \log _{10}\left\langle\left|\frac{\Psi}{\Psi_{\text {ref }}}\right|^{2}\right\rangle-S L-T L A
$$


where $\Psi$ is the scattered field, $\Psi_{r e f}=1 \mu \mathrm{Pa}$ is the reference acoustic pressure in water, $S L$ is the source level, TLA is the depth-averaged two-way transmission loss to individual scatterers integrated over OAWRS imagining resolution, given [26] by

$$
T L A=10 \log _{10}\left(\int_{A_{R}\left(\rho_{C}\right)} \frac{1}{H} \int_{z_{0}-H / 2}^{z_{0}+H / 2} \chi\left(\boldsymbol{r}, \boldsymbol{r}_{\mathbf{0}}, \boldsymbol{r}_{\boldsymbol{T}}\right) d z_{T} d \boldsymbol{\rho}_{\boldsymbol{T}}^{\mathbf{2}} / r_{r e f}^{-2}\right)
$$

where $\chi\left(\boldsymbol{r}, \boldsymbol{r}_{\mathbf{0}}, \boldsymbol{r}_{\boldsymbol{T}}\right)$ is the magnitude squared of the two-way Green function from source $\boldsymbol{r}_{\mathbf{0}}$ to target $\boldsymbol{r}_{\boldsymbol{T}}$ to receiver $\boldsymbol{r}$, given by

$$
\chi\left(\boldsymbol{r}, \boldsymbol{r}_{\mathbf{0}}, \boldsymbol{r}_{\boldsymbol{T}}\right)=(4 \pi)^{4}\left\langle\left|G\left(\boldsymbol{r} \mid \boldsymbol{r}_{\boldsymbol{T}} ; f, c\left(\boldsymbol{r}_{\boldsymbol{w}}\right), d\left(\boldsymbol{r}_{\boldsymbol{w}}\right)\right)\right|^{2}\left|G\left(\boldsymbol{r}_{\boldsymbol{T}} \mid \boldsymbol{r}_{\mathbf{0}} ; f, c\left(\boldsymbol{r}_{\boldsymbol{w}}\right), d\left(\boldsymbol{r}_{\boldsymbol{w}}\right)\right)\right|^{2} \mid \boldsymbol{r}_{\boldsymbol{T}}\right\rangle
$$

where $G\left(\boldsymbol{r} \mid \boldsymbol{r}_{\boldsymbol{T}} ; f, c\left(\boldsymbol{r}_{\boldsymbol{w}}\right), d\left(\boldsymbol{r}_{\boldsymbol{w}}\right)\right)$ is the Green function between the target location $\boldsymbol{r}_{\boldsymbol{T}}=$ $\left(\boldsymbol{\rho}_{\boldsymbol{T}}, z_{T}\right)$ and the receiver location $\boldsymbol{r}, \boldsymbol{\rho}_{\boldsymbol{T}}=\left(x_{T}, y_{T}\right)$ is the horizontal target location, $G\left(\boldsymbol{r}_{\boldsymbol{T}} \mid \boldsymbol{r}_{\mathbf{0}} ; f, c\left(\boldsymbol{r}_{\boldsymbol{w}}\right), d\left(\boldsymbol{r}_{\boldsymbol{w}}\right)\right)$ is the Green function between the source location $\boldsymbol{r}_{\mathbf{0}}=\left(\boldsymbol{\rho}_{\boldsymbol{S}}, z_{S}\right)$ and the target location $\boldsymbol{r}_{\boldsymbol{T}}, c\left(\boldsymbol{r}_{\boldsymbol{w}}\right)$ and $d\left(\boldsymbol{r}_{\boldsymbol{w}}\right)$ are the sound speed and density of any point $r_{w}$ in the propagation path, respectively. While $\chi\left(\boldsymbol{r}, \boldsymbol{r}_{\mathbf{0}}, \boldsymbol{r}_{\boldsymbol{T}}\right)$ includes transmission losses due to spreading and seafloor attenuation, it does not include losses due to attenuation from fish. A parabolic equation model [9] is used to calculate the Green functions in a rangedependent environment. The conditional expectation over the sound speed is determined by averaging five Monte-Carlo realizations, where the Green functions are calculated along the propagation path in range and depth for each realization. Each Monte-Carlo realization employs sound-speed profiles measured during the 2014 OAWRS experiment (Appendix F of [15]) every $500 \mathrm{~m}$ along the propagation path [1].

In cases where there is significant attenuation from fish (Figure 3-3), scattering strength uncorrected for attenuation from fish $(\widetilde{S S})$ can be calculated from

$$
\widetilde{S S} \equiv S S-\Delta S P L_{2 w a y}=10 \log _{10}\left\langle\left|\frac{\Psi}{\Psi_{r e f}}\right|^{2}\right\rangle-S L-T L A
$$

where $\triangle S P L_{2 w a y}$ is the decrease in sound pressure level due to two-way attenuation from fish. Measurements of fish-attenuated scattering strength can be corrected using the method described in Appendix I.

Since attenuation from fish prevented the entire region from between instantaneously surveyed by OAWRS, the areal population density map in Figure 4-5 is generated from five 
instantaneous OAWRS transmissions between 3:00 and 6:00 on February 21, 2014 (3:04:09, 3:24:09, 4:19:09, 5:04:09, and 5:39:59). Discrepancies between the overlapping OAWRS scattering strength maps are assumed to be caused by attenuation from fish, and the maximum scattering strength between overlapping pixels was chosen in order to minimize the effects of attenuation, according to:

$$
S S(\boldsymbol{r})=\max _{j}\left[\widetilde{S S}_{j}(\boldsymbol{r})\right]
$$

where $S S(\boldsymbol{r})$ is the estimated scattering strength at horizontal position $\boldsymbol{r}$ and $\widetilde{S S}(\boldsymbol{r})$ is the fish-attenuated scattering strength measured by OAWRS transmission $j$. The areal population density of herring $n_{A}$ is then calculated according $[37,38,26,19]$ to

$$
10 \log _{10}\left(n_{A}\right)=S S(\boldsymbol{r})-T S
$$

where $T S=-29.5$ is the calibrated target strength of herring measured during this experiment at sensing frequency $f=955 \mathrm{~Hz}$ [15]. A running circular averaging window of 1 $\mathrm{km}$ radius is applied to the composite population density map in order to eliminate boundary discontinuities due to lack of temporal simultaneity of the five instantaneous OAWRS images.

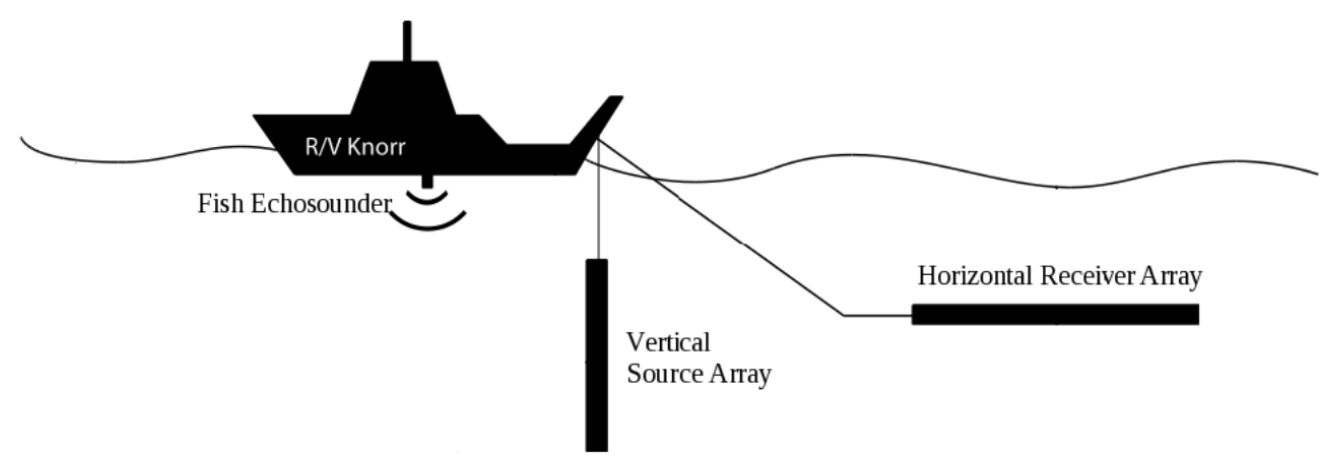

Seafloor

Figure A-1: OAWRS system used for herring measurements during the Nordic Seas 2014 Experiment [35]. The system is effectively monostatic with source and receiver arrays towed from the same research vessel (RV Knorr). The OAWRS source was developed under the National Science Foundation and Sloan Foundation MRI program for wide-area sensing of marine life, and the ONR Five Octave Research Array (FORA) was used as the OAWRS receiver. 


\section{Appendix B}

\section{Evaluating whether Attenuation Effects are Present}

Attenuation from Ålesund herring is observable when an occluding shoal moves into the propagation path to a distant shoal and the scattered returns from the distant shoal are diminished (Figure 2-1). The presence of attenuation is further confirmed by the steady decrease in scattering strength within the same occluding shoal as range increases (Figure B-1B). We do not see evidence of attenuation from Gulf of Maine herring, Finnmark capelin, and Lofoten cod since there is not a noticeable decrease in scattering strength within a shoal as range increases (Figures B-1D, B-1F, B-1H). These observations are consistent with the theoretical results shown in Figure 2-5, which predict significant attenuation from Ålesund herring and no significant attenuation from Gulf of Maine herring, Finnmark capelin, and Lofoten cod at the sensing frequencies used in the OAWRS experiments. 

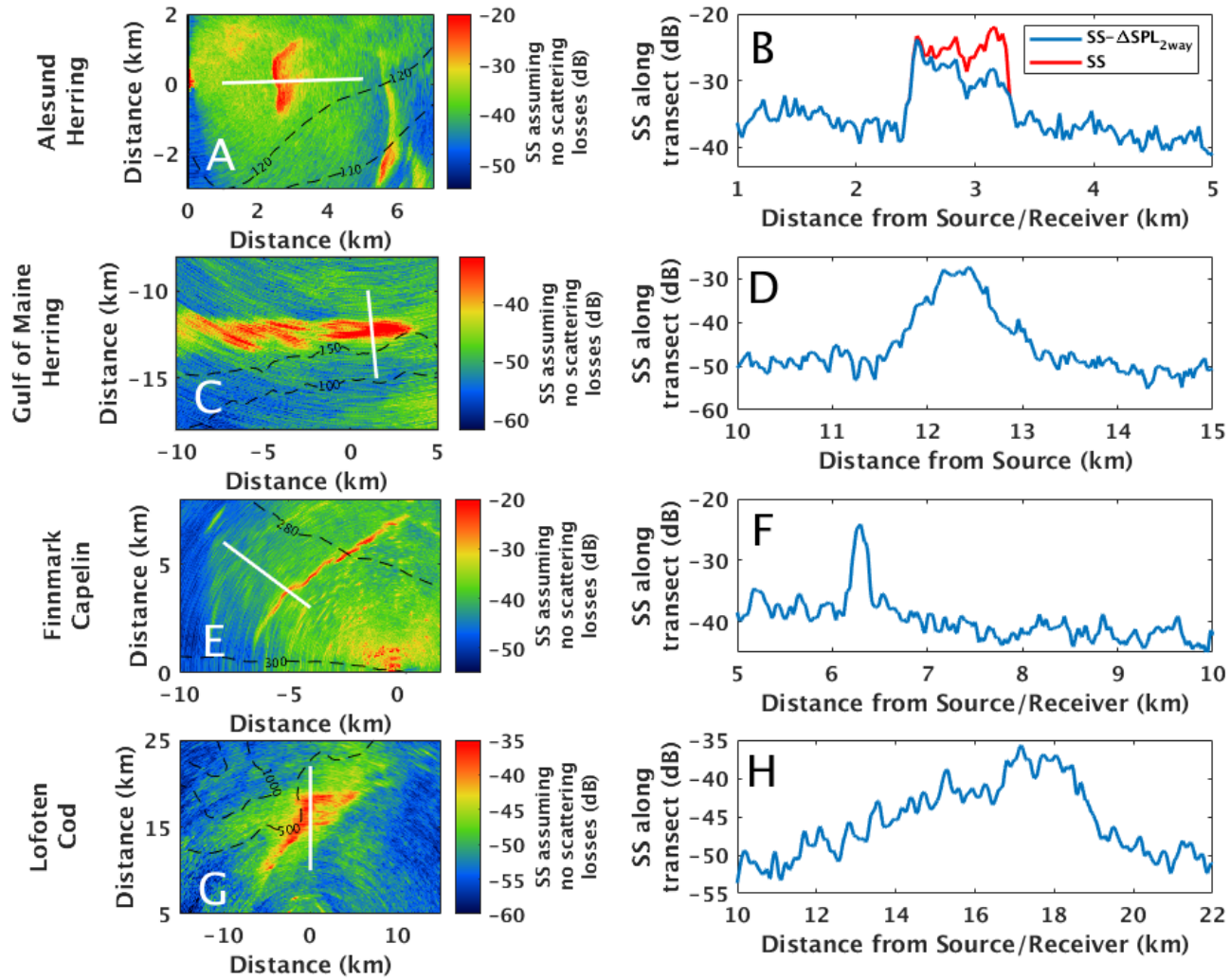

Figure B-1: Example OAWRS scattering strength images of fish shoals assuming no scattering losses are shown from four seperate continental shelf environments (A, C, E, and G). Scattering strength levels along radial transects that bisect fish shoals are shown in $\mathrm{B}, \mathrm{D}$, $\mathrm{F}$, and $\mathrm{H}$. The positions of the radial transects are given by white lines in A, C, E, and G. We see evidence of attenuation through Ålesund herring shoals (blue line in B) since there is a sharp increase in scattering strength where the shoal begins $(2.5 \mathrm{~km}$ from the source/receiver) followed by a steady decease in scattering strength caused by attenuation as range increases. After applying the attenuation correction described in Section 2.3.3 this effect is no longer present (red line in B). We do not see evidence of attenuation from Gulf of Maine herring, Finnmark capelin, and Lofoten cod (D, F, and H, respectively) since there is no steady decrease in scattering strength as range increases, and no attenuation correction is necessary. Sensing frequencies for the scattering strength images shown here are $955 \mathrm{~Hz}$ for Ålesund herring, $950 \mathrm{~Hz}$ for Gulf of Maine herring, $955 \mathrm{~Hz}$ for Lofoten cod, and $1335 \mathrm{~Hz}$ for Finnmark capelin. Black dotted lines indicate water depth contours. 


\section{Appendix C}

\section{Modeling the Scatter Function of Individual Fish}

The target strength of an individual fish in a shoal with mean depth $z_{0}$ and vertical thickness $H$ is determined [26] by

$$
T S=10 \log _{10}\left(\frac{1}{H} \int_{z_{0}-H / 2}^{z_{0}+H / 2} \int_{l}\left|\frac{S}{k}\right|^{2} g(l) d l d z\right)
$$

where $k$ is the wavenumber, $l$ is the fork length of an individual fish, $g(l)$ is the Gaussian probability density function of the fork length, $z$ is the fish depth, and $S$ is the far-field scatter function of an individual fish, given [6] by:

$$
S\left(z, z_{n b}, l, f\right)=\frac{\left(\frac{f_{0}^{2}\left(z, z_{n b}, l\right)}{f^{2}}-1\right) k \bar{a}\left(z, z_{n b}, l\right)}{\left(\frac{f_{0}^{2}\left(z, z_{n b}, l\right)}{f^{2}}-1\right)^{2}+\delta^{2}\left(z, z_{n b}, l, f\right)}+i \frac{\delta\left(z, z_{n b}, l, f\right) k \bar{a}\left(z, z_{n b}, l\right)}{\left(\frac{f_{0}^{2}\left(z, z_{n b}, l\right)}{f^{2}}-1\right)^{2}+\delta^{2}\left(z, z_{n b}, l, f\right)}
$$

where $k$ is the wavenumber, $f$ is the sensing frequency, $f_{0}\left(z, z_{n b}, l\right)$ is the resonant frequency of swimbladder, $\bar{a}\left(z, z_{n b}, l\right)$ is the equivalent swimbladder radius, and $\delta\left(z, z_{n b}, l, f\right)$ is a dimensionless damping coefficient. The equivalent swimbladder radius is determined [28] by

$$
\bar{a}\left(z, z_{n b}, l\right)=\left[\frac{3}{4 \pi} \frac{c_{n b} m_{\text {flesh }}(l)}{\rho_{\text {flesh }}} \frac{1+z_{n b} / 10}{1+z / 10}\right]^{1 / 3}
$$

assuming that the swimbladder volume varies with pressure according to Boyle's law, where $c_{n b}$ is the ratio of the swimbladder volume at neutral buoyancy to the volume of the fish flesh $V_{\text {flesh }}=m_{\text {flesh }}(l) / \rho_{\text {flesh }} . m_{\text {flesh }}(l)$ is the mass of a single fish empirically determined by the fork length $l[19]$, and $\rho_{\text {flesh }}$ is the density of the fish flesh. The resonance frequency 
of the swimbladder is determined by

$$
f_{0}\left(z, z_{n b}, l\right)=\frac{\kappa\left(\epsilon\left(z, z_{n b}, l\right)\right)}{2 \pi r\left(z, z_{n b}\right)} \sqrt{\frac{3 \gamma P_{a t m}(1+z / 10)}{\rho_{\text {flesh }}}}
$$

where $\gamma=1.4$ is the ratio of the specific heats of air, and $P_{a t m}=1.013 \mathrm{~Pa}$ is the atmospheric pressure. The correction term $\kappa\left(\epsilon\left(z, z_{n b}, l\right)\right)$ is a function of $\epsilon\left(z, z_{n b}, l\right)$, the swimbladder's eccentricity. The correction term $\kappa\left(\epsilon\left(z, z_{n b}, l\right)\right)$ for a prolate spheroidal swimbladder is given [60] by:

$$
\kappa\left(\epsilon\left(z, z_{n b}, l\right)\right)=\frac{\sqrt{2}\left(1-\epsilon^{2}\left(z, z_{n b}, l\right)\right)^{1 / 4}}{\epsilon^{1 / 3}\left(z, z_{n b}, l\right)}\left[\ln \left(\frac{1+\sqrt{1+\epsilon^{2}\left(z, z_{n b}, l\right)}}{1-\sqrt{1-\epsilon^{2}\left(z, z_{n b}, l\right)}}\right)\right]^{-1 / 2}
$$

where $\epsilon\left(z, z_{n b}, l\right)$ is the ratio of the minor to major axis of a prolate spherical swimbladder given by $\epsilon\left(z, z_{n b}, l\right)=\left(\frac{c_{s b} l}{\bar{a}\left(z, z_{n b}, l\right)}\right)^{-3 / 2}$, and $c_{s b}$ is the ratio of the major axis of the swimbladder to the fish fork length $l[19]$.

The dimensionless damping coefficient $\delta\left(z, z_{n b}, l, f\right)$ in Eq. A2 is obtained from the sum of radiation damping $\delta_{\text {rad }}$ and viscous damping $\delta_{v i s}$ :

$$
\delta\left(z, z_{n b}, l, f\right)=\delta_{r a d}+\delta_{v i s}=\frac{2 \pi f \bar{a}\left(z, z_{n b}, l\right)}{c}+\frac{\xi_{f}}{\pi \bar{a}^{2}\left(z, z_{n b}, l\right) f \rho_{f}}
$$

where $f$ is the frequency, $c$ is the sound speed, $\xi_{f}$ is the viscosity of the fish flesh, and $\rho_{f}$ is the density of fish flesh [2]. 


\section{Appendix D}

\section{Modeling Two-Way Attenuation in a Waveguide Environment}

The two-way scattered field without attenuation from a single scatterer at $\boldsymbol{r}_{\boldsymbol{t}}=\left(\boldsymbol{\rho}_{\boldsymbol{t}}, z_{t}\right)$ with scatter function $S$ is given by

$$
\Psi_{i, 2 w a y}\left(\boldsymbol{r} \mid \boldsymbol{r}_{\mathbf{0}}\right)=\Psi_{i}\left(\boldsymbol{r}_{\boldsymbol{t}} \mid \boldsymbol{r}_{\mathbf{0}}\right) \frac{S}{k} \Psi_{i}\left(\boldsymbol{r} \mid \boldsymbol{r}_{\boldsymbol{t}}\right)
$$

where $\Psi_{i}\left(\boldsymbol{r}_{\boldsymbol{t}} \mid \boldsymbol{r}_{\mathbf{0}}\right)$ is the incident field from source $\boldsymbol{r}_{\mathbf{0}}$ to target $\boldsymbol{r}_{\boldsymbol{t}}$ and $\Psi_{i}\left(\boldsymbol{r} \mid \boldsymbol{r}_{\boldsymbol{t}}\right)$ is the field scattered from target $\boldsymbol{r}_{\boldsymbol{t}}$ to receiver $\boldsymbol{r}$, as defined in Eq. 2.1. Note that $\Psi_{i}\left(\boldsymbol{r}_{\boldsymbol{t}} \mid \boldsymbol{r}_{\mathbf{0}}\right)$ and $\Psi_{i}\left(\boldsymbol{r} \mid \boldsymbol{r}_{\boldsymbol{t}}\right)$ assume no losses due to scatterers between $\boldsymbol{r}_{\mathbf{0}}$ and $\boldsymbol{r}_{\boldsymbol{t}}$ or between $\boldsymbol{r}_{\boldsymbol{t}}$ and $\boldsymbol{r}$. For multiple individual scatterers with scatter function $S$, the scattered field then becomes

$$
\Psi_{i, 2 w a y}\left(\boldsymbol{r} \mid \boldsymbol{r}_{\mathbf{0}}\right)=\sum_{\boldsymbol{r}_{\boldsymbol{t}}} \Psi_{i}\left(\boldsymbol{r}_{\boldsymbol{t}} \mid \boldsymbol{r}_{\mathbf{0}}\right) \frac{S}{k} \Psi_{i}\left(\boldsymbol{r} \mid \boldsymbol{r}_{\boldsymbol{t}}\right)
$$

Since the field is fully randomized in a ocean waveguide environment [34], the intensity can be written as

$$
\left|\Psi_{i, 2 w a y}\left(\boldsymbol{r} \mid \boldsymbol{r}_{\mathbf{0}}\right)\right|^{2}=\sum_{\boldsymbol{r}_{\boldsymbol{t}}}\left|\Psi_{i}\left(\boldsymbol{r}_{\boldsymbol{t}} \mid \boldsymbol{r}_{\mathbf{0}}\right)\right|^{2}\left|\frac{S}{k}\right|^{2}\left|\Psi_{i}\left(\boldsymbol{r} \mid \boldsymbol{r}_{\boldsymbol{t}}\right)\right|^{2}
$$

For a uniform distribution of scatterers of scatter function $S$ with mean depth $z_{0}$, height $H$, and volume number density $n_{V}$, the intensity of the scattered field without attenuation at resolution footprint $A_{R}\left(\rho_{C}\right)$ can then be written as

$$
\left|\Psi_{i, 2 \text { way }}\left(\boldsymbol{r} \mid \boldsymbol{r}_{\mathbf{0}}\right)\right|^{2}=\left|\frac{S}{k}\right|^{2} n_{V} \int_{A_{R}\left(\rho_{C}\right)} \int_{z=z_{0}-H / 2}^{z=z_{0}+H / 2}\left|\Psi_{i}\left(\boldsymbol{r}_{\boldsymbol{t}} \mid \boldsymbol{r}_{\mathbf{0}}\right)\right|^{2}\left|\Psi_{i}\left(\boldsymbol{r} \mid \boldsymbol{r}_{\boldsymbol{t}}\right)\right|^{2} d z_{t} d \boldsymbol{\rho}_{\boldsymbol{t}}^{\mathbf{2}}
$$


Similarly, the mean intensity of the scattered field including attenuation can be written as

$$
\left\langle\left|\Psi_{T, 2 w a y}\left(\boldsymbol{r} \mid \boldsymbol{r}_{\mathbf{0}}\right)\right|^{2}\right\rangle=\left|\frac{S}{k}\right|^{2} n_{V} \int_{A_{R}\left(\rho_{C}\right)} \int_{z=z_{0}-H / 2}^{z=z_{0}+H / 2}\left\langle\left|\Psi_{T}\left(\boldsymbol{r}_{\boldsymbol{t}} \mid \boldsymbol{r}_{\mathbf{0}}\right)\right|^{2}\right\rangle\left\langle\left|\Psi_{T}\left(\boldsymbol{r} \mid \boldsymbol{r}_{\boldsymbol{t}}\right)\right|^{2}\right\rangle d z_{t} d \boldsymbol{\rho}_{\boldsymbol{t}}^{\mathbf{2}}
$$

where $\left\langle\left|\Psi_{T}\left(\boldsymbol{r}_{\boldsymbol{t}} \mid \boldsymbol{r}_{\mathbf{0}}\right)\right|^{2}\right\rangle$ is the total mean intensity from source $\boldsymbol{r}_{\mathbf{0}}$ to target $\boldsymbol{r}_{\boldsymbol{t}}$ and $\left\langle\left|\Psi_{T}\left(\boldsymbol{r} \mid \boldsymbol{r}_{\boldsymbol{t}}\right)\right|^{2}\right\rangle$ is the total mean intensity from target $\boldsymbol{r}_{\boldsymbol{t}}$ to receiver $\boldsymbol{r}$, as defined in Eq. 2.9. Note that $\left\langle\left|\Psi_{T}\left(\boldsymbol{r}_{\boldsymbol{t}} \mid \boldsymbol{r}_{\mathbf{0}}\right)\right|^{2}\right\rangle$ and $\left\langle\left|\Psi_{T}\left(\boldsymbol{r} \mid \boldsymbol{r}_{\boldsymbol{t}}\right)\right|^{2}\right\rangle$ take into account losses from scatterers between $\boldsymbol{r}_{\mathbf{0}}$ and $\boldsymbol{r}_{\boldsymbol{t}}$ and between $\boldsymbol{r}_{\boldsymbol{t}}$ and $\boldsymbol{r}$, respectively. Here we assume that the intensities $\left|\Psi_{T}\left(\boldsymbol{r}_{\boldsymbol{t}} \mid \boldsymbol{r}_{\mathbf{0}}\right)\right|^{2}$ and $\left|\Psi_{T}\left(\boldsymbol{r} \mid \boldsymbol{r}_{\boldsymbol{t}}\right)\right|^{2}$ are uncorrelated since the field is fully randomized in a ocean waveguide environment.

The decrease in sound pressure level due to attenuation during two-way propagation can then be defined as

$$
\begin{aligned}
\Delta S P L_{2 w a y} & =10 \log _{10}\left|\Psi_{i, 2 w a y}\left(\boldsymbol{r} \mid \boldsymbol{r}_{\mathbf{0}}\right)\right|^{2}-10 \log _{10}\left(\left\langle\left|\Psi_{T, 2 w a y}\left(\boldsymbol{r} \mid \boldsymbol{r}_{\mathbf{0}}\right)\right|^{2}\right\rangle\right) \\
& =10 \log _{10}\left(\int_{A_{R}\left(\rho_{C}\right)} \int_{z=z_{0}-H / 2}^{z=z_{0}+H / 2}\left|\Psi_{i}\left(\boldsymbol{r}_{\boldsymbol{t}} \mid \boldsymbol{r}_{\mathbf{0}}\right)\right|^{2}\left|\Psi_{i}\left(\boldsymbol{r} \mid \boldsymbol{r}_{\boldsymbol{t}}\right)\right|^{2} d z_{t} d \boldsymbol{\rho}_{\boldsymbol{t}}^{\mathbf{2}}\right) \\
& -10 \log _{10}\left(\int_{A_{R}\left(\rho_{C}\right)} \int_{z=z_{0}-H / 2}^{z=z_{0}+H / 2}\left\langle\left|\Psi_{T}\left(\boldsymbol{r}_{\boldsymbol{t}} \mid \boldsymbol{r}_{\mathbf{0}}\right)\right|^{2}\right\rangle\left\langle\left|\Psi_{T}\left(\boldsymbol{r} \mid \boldsymbol{r}_{\boldsymbol{t}}\right)\right|^{2}\right\rangle d z_{t} d \boldsymbol{\rho}_{\boldsymbol{t}}^{\mathbf{2}}\right)
\end{aligned}
$$




\section{Appendix E}

\section{Derivation of Log-Likelihood Function}

Given correlated Gaussian variables $X$ and $Y$ with respective means $\mu_{X}$ and $\mu_{Y}$ dependent on parameter $\theta$ and respective variances $\sigma_{X}^{2}$ and $\sigma_{Y}^{2}$ independent of the mean, the joint probability density of $X$ and $Y$ can be written as:

$$
p(X, Y \mid \theta)=\frac{1}{2 \pi \sigma_{X} \sigma_{Y} \sqrt{1-\rho_{X, Y}^{2}}} \exp \left[\frac{z(\theta)}{2\left(1-\rho_{X, Y}^{2}\right)}\right]
$$

where

$$
z(\theta)=-\frac{\left(X-\mu_{X}(\theta)\right)^{2}}{\sigma_{X}^{2}}-\frac{\left(Y-\mu_{Y}(\theta)\right)^{2}}{\sigma_{Y}^{2}}+\frac{2 \sigma_{X, Y}\left(X-\mu_{X}(\theta)\right)\left(Y-\mu_{Y}(\theta)\right)}{\sigma_{X}^{2} \sigma_{Y}^{2}}
$$

and the correlation coefficient $\rho_{X, Y}$ between $X$ and $Y$ is defined as

$$
\rho_{X, Y}=\frac{\sigma_{X, Y}}{\sigma_{X} \sigma_{Y}}
$$

where $\sigma_{X, Y}$ is the covariance between $X$ and $Y$.

The logarithm of the probability density function will then be:

$$
\log (p(X, Y \mid \theta))=-\log \left(2 \pi \sigma_{X} \sigma_{Y} \sqrt{1-\rho_{X, Y}^{2}}\right)+\frac{z(\theta)}{2\left(1-\rho_{X, Y}^{2}\right)}
$$

By excluding terms that are not dependent on $\theta$, and we are left with the log-likelihood function:

$$
\ell(\theta)=z(\theta)
$$




\section{Appendix $\mathrm{F}$}

\section{Sound Speed Profiles}

Water-column sound speed profiles are shown in Figure F-1. 

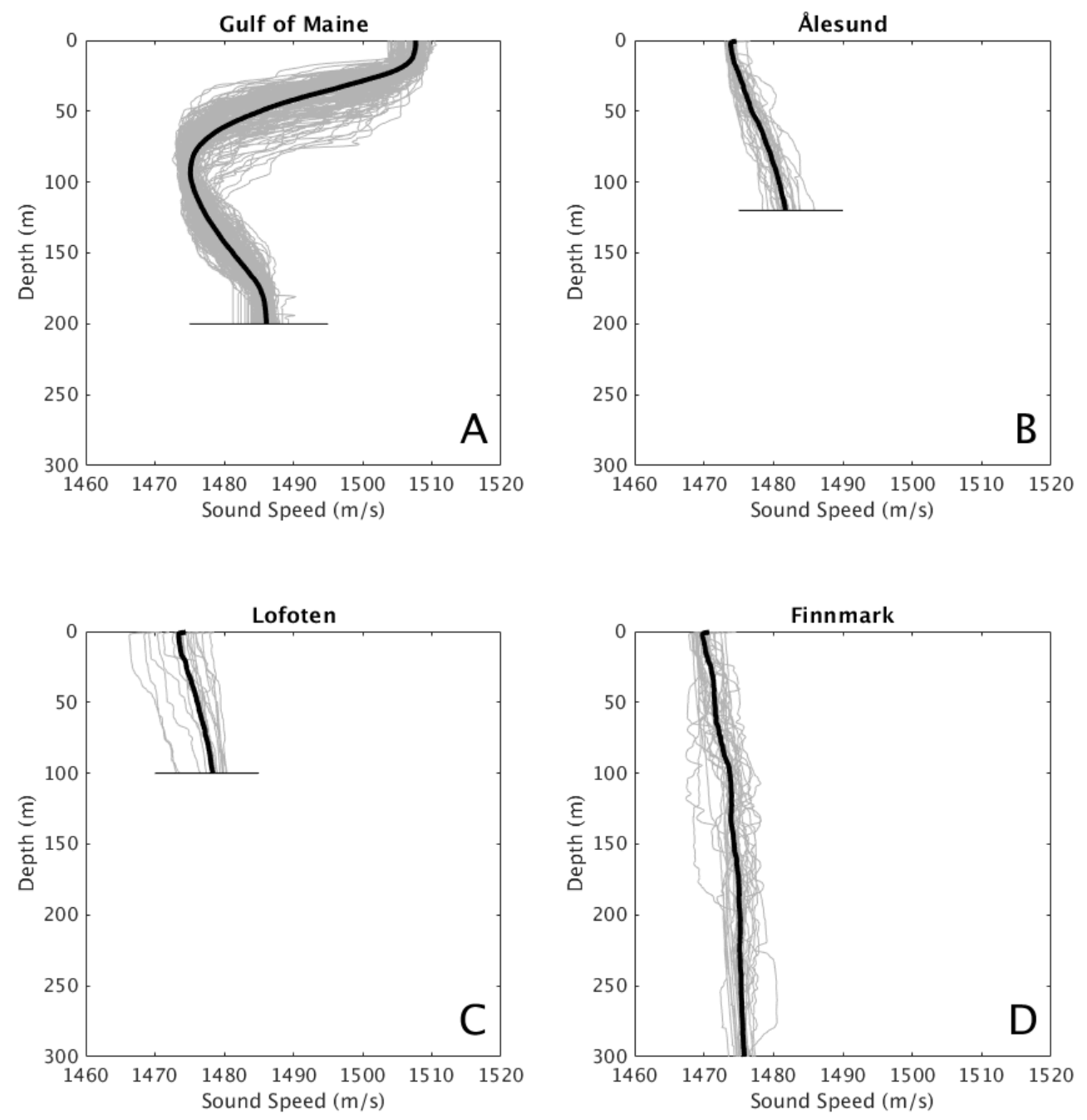

Figure F-1: Profiles of water-column sound speed from XBT measurements in the Gulf of Maine (A), Ålesund waters (B), Lofoten waters (C), and Finnmark waters (D). 


\section{Appendix G}

\section{Calibration of Target Strength}

Scattering strength is converted to areal population density by calibration with local in situ measurements of areal population density obtained from echosounder measurements. This calibration is performed using OAWRS images of the large, elongated herring shoal observed on February 21 (Figure G-1A) with a nearly concurrent echosounder transect (Figure G1B). Areal density $n_{A}$ at range $r$ along the transect is calculated according to Equation A1. Target strength measured by OAWRS at frequency $f_{i}$ and range $r$ along the transect is calculated according to:

$$
T S_{\text {data }}\left(f_{i}, r\right)=10 \log _{10}\left(10^{\left(S S_{\text {data }}\left(f_{i}, r\right)-10 \log _{10}\left(n_{A}(r)\right)\right) / 10}\right)
$$

where $S S_{\text {data }}\left(f_{i}, r\right)$ is the scattering strength measured by OAWRS at range $r$ along the transect and sensing frequency $f_{i}$ (Appendix A). Neutral buoyancy depth $z_{n b}$ is then determined by modeling target strength $T S_{\text {model }}\left(z_{n b}, f_{i}\right)$ according to Appendix $\mathrm{C}$ assuming the measured distribution of herring depths (Figure G-1C) and maximizing the following likelihood function:

$$
\ell\left(z_{n b}\right)=\sum_{i=1}^{N_{f}}\left(\frac{-\left(T S_{\text {model }}\left(z_{n b}, f_{i}\right)-\left\langle T S_{\text {data }}\left(f_{i}\right)\right\rangle\right)}{\sigma_{T S}\left(f_{i}\right)^{2}}\right)
$$

where $\left\langle T S_{\text {data }}\left(f_{i}\right)\right\rangle$ is the mean target strength measured along the transect at frequency $f_{i}$, $\sigma_{T S}\left(f_{i}\right)$ is the standard deviation at frequency $f_{i}$, and $N_{f}$ is the number of frequencies. The likelihood function is maximized by searching for across possible neutral buoyancy depths from sea surface to seafloor, and calibrated neutral buoyancy depth is determined to be $z_{n b}$ 
$=3$ meters below the sea surface (Figure G-1D).

OAWRS scattering strength maps are then converted to areal population density maps according to:

$$
10 \log _{10}\left(n_{A}(\rho, \theta)=S S(\rho, \theta)+T S\left(z_{n b}, f\right)\right.
$$

where $n_{A}$ is the areal population density at range $\rho$ and azimuth $\theta, S S$ is the OAWRS scattering strength, $f=955$ is the frequency of the OAWRS transmissions shown here, and $z_{n b}$ is the calibrated neutral buoyancy depth. 

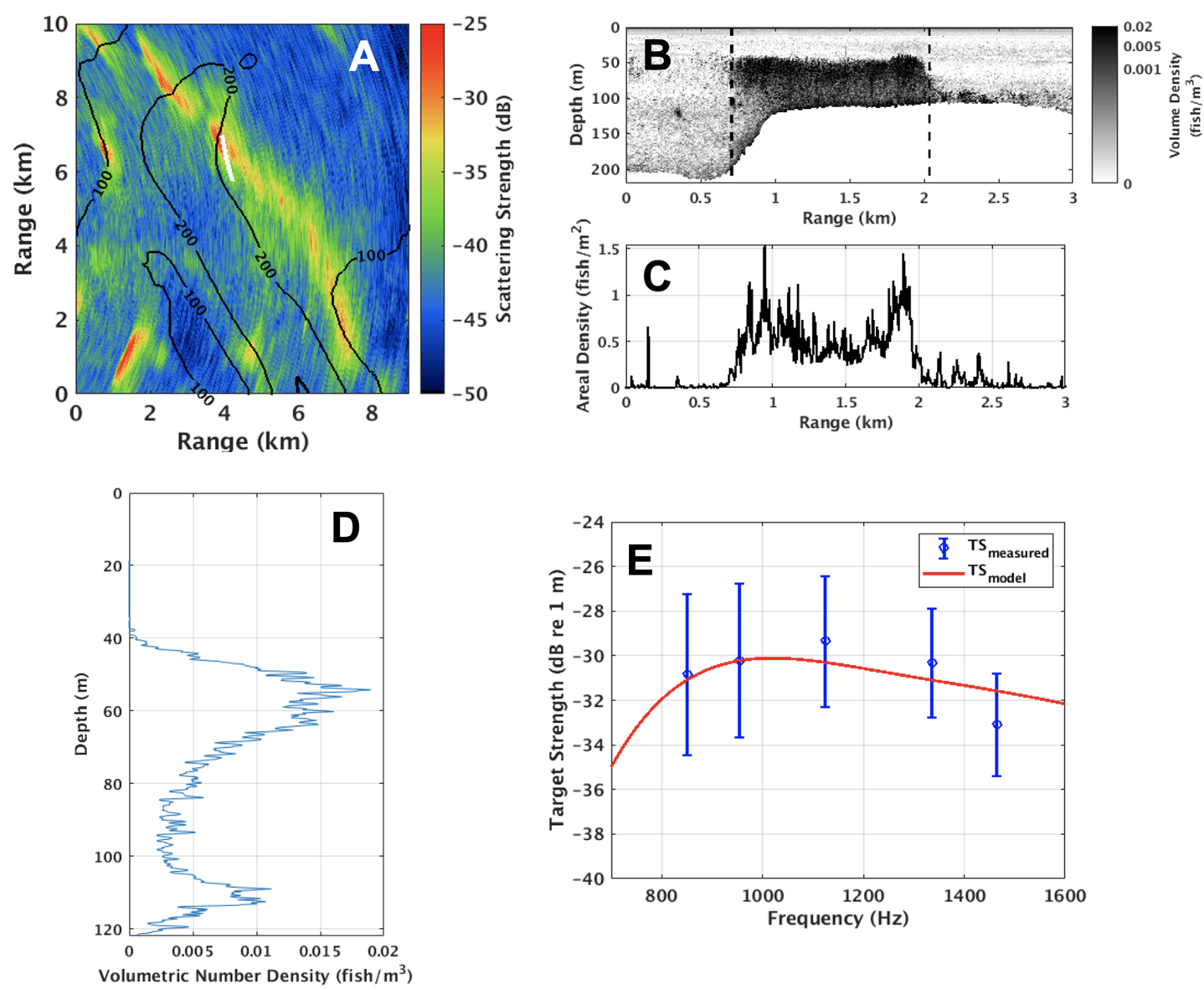

Figure G-1: The target strength of herring in Ålesund is determined by calibrating OAWRS scattering strength measurements of a large, elongated herring shoal observed on February 21 with nearly concurrent echosounder transects. The OAWRS scattering strength map from February 21, 2014 at 3:26:39 at sensing frequency $955 \mathrm{~Hz}$ is shown in (A) with the echosounder transect from 2:52:00-2:58:00 overlain in white. Echosounder data is shown in (B), where vertical black dotted lines denote the transect studied here. The average areal population density along the echosounder transect is shown in (C), and average volume density of herring with respect to depth along this transect is shown in (D). The target strength of herring in this shoal is modeled assuming the depth distribution shown in (D), and neutral buoyancy depth is determined to be $3 \mathrm{~m}$ by performing a least-squares fit between measured and modeled target strength (E). 


\section{Appendix $\mathrm{H}$}

\section{Measuring Sensing Range}

Sensing range $\rho_{\text {sens }}$ is measured as the maximum range $\rho$ at which the azimuthally-intensityaveraged-sound pressure level $S P L_{A}$ is greater than the attenuated ambient noise over the frequency band of the signal by more than the detection threshold $D T$ :

$$
\rho_{\text {sens }}=\max \left[\rho \mid S P L_{A}(\rho)>N L-\Delta N L+D T\right]
$$

where $N L$ is the ambient noise level and $\Delta N L$ is the reduction in ambient noise due to attenuation from fish over the frequency band of the signal. Here the detection threshold $D T$ is set at $5.6 \mathrm{~dB}$, which is the standard deviation of an acoustic measurement after saturated multipath propagation when the time-bandwidth product is one [16, 34]. The azimuthallyintensity-averaged-sound pressure level $S P L_{A}(\rho)$ is calculated by averaging the intensity of the beamformed scattered field across azimuthal angle $\theta$ over multiple transmissions within the relevant time frame:

$$
S P L_{A}(\rho) \equiv 10 \log _{10}\left\langle\left|\frac{\Psi(\rho)}{\Psi_{r e f}}\right|^{2}\right\rangle=10 \log _{10}\left(\frac{1}{N} \sum_{k=1}^{N} \frac{1}{\theta_{\max }-\theta_{\min }} \int_{\theta_{\min }}^{\theta_{\max }}\left|\frac{\Psi^{(k)}(\rho, \theta)}{\Psi_{r e f}}\right|^{2} d \theta\right)
$$

where $\Psi^{(k)}(\rho, \theta)$ is the beamformed scattered field from transmission $k$ at range $\rho$ and azimuthal angle $\theta, N$ is the number of transmissions within the time frame analyzed, $\Psi_{r e f}=$ $1 \mu \mathrm{Pa}$ is the reference pressure for underwater sound, $\left\langle|\Psi(\rho)|^{2}\right\rangle$ is the magnitude squared of the beamformed scattered field averaged over $\theta$ and $k$, and the limits of integration $\theta_{\text {min }}$ and $\theta_{\max }$ are chosen to exclude beams within $25^{\circ}$ of endfire.

Attenuated ambient noise over the frequency band of the signal $(N L-\Delta N L)$ is measured 
from the average acoustic intensity above a minimum range $\rho_{\min }$ :

$$
N L-\Delta N L=10 \log _{10}\left(\frac{1}{\rho_{\max }-\rho_{\min }} \int_{\rho_{\min }}^{\rho_{\max }}\left\langle\left|\frac{\Psi(\rho)}{\Psi_{\text {ref }}}\right|^{2}\right\rangle d \rho\right)
$$

where $\rho_{\max }=33 \mathrm{~km}$ is the maximum recorded range, determined by the recording time for each OAWRS transmission, and $\rho_{\min }$ is the range after which we can safely say that ambient noise dominates the received sound pressure level for each OAWRS transmission. $\rho_{\min }$ is chosen so that the recorded sound pressure level between $\rho_{\min }$ and $\rho_{\max }$ does not significantly change with range, indicating that the measured sound pressure level is dominated by ambient noise. This is quantified by expressing $\rho_{\min }$ as:

$$
\rho_{\text {min }}=\min \left[\rho_{\min }^{\prime}\left|\frac{1}{N} \sum_{k=1}^{N}\right| \beta^{(k)}\left(\rho_{\text {min }}^{\prime}\right) \mid<\beta_{\text {threshold }}\right]
$$

where $\beta^{(k)}\left(\rho_{\min }\right)$ is the slope of the linear regression of sound pressure level with respect to range for transmission $k$ between $\rho_{\min }$ and $\rho_{\max }$, given by:

$$
\beta^{(k)}\left(\rho_{\min }^{\prime}\right)=\frac{\int_{\rho_{\min }^{\prime}}^{\rho_{\max }}\left(\left(S P L_{A}^{(k)}(\rho)-\int_{\rho_{\min }^{\prime}}^{\rho_{\max }} S P L_{A}^{(k)}(\rho) d \rho\right)\left(\rho-\frac{1}{2}\left(\rho_{\max }-\rho_{\min }^{\prime}\right)\right) d \rho\right.}{\int_{\rho_{\min }^{\prime}}^{\rho_{\max }}\left(\rho-\frac{1}{2}\left(\rho_{\max }-\rho_{\min }^{\prime}\right)\right) d \rho}
$$

where $S P L_{A}^{(k)}(\rho)$ is the range-dependent sound pressure level for a single transmission $k$, and $\beta_{\text {threshold }}$ is the maximum slope at which we determine the sound pressure level to be sufficiently flat with respect to range. Setting the slope threshold at $\beta_{\text {threshold }}=0.01$ $\mathrm{dB} / \mathrm{km}, \rho_{\min }$ is calculated as $29.5 \mathrm{~km}$. Visual inspection of Figures H-1C-D confirms that measured sound pressure level flattens with respect to range above $29.5 \mathrm{~km}$.

Combining Equations A1 and A3 yields the following formulation for measuring sensing range:

$$
\rho_{\text {sens }}=\max \left[\rho \mid S P L_{A}(\rho)>10 \log _{10}\left(\frac{1}{\rho_{\max }-\rho_{\min }} \int_{\rho_{\min }}^{\rho_{\max }}\left\langle\left|\frac{\Psi(\rho)}{\Psi_{\text {ref }}}\right|^{2}\right\rangle d \rho\right)+D T\right]
$$

Sensing range at azimuthal angle $\theta$ for OAWRS transmission $k$ can be similarly defined 
as:

$\rho_{\text {sens }}^{(k)}(\theta)=\max \left[\rho \mid S P L_{A}^{(k)}(\rho, \theta)>10 \log _{10}\left(\frac{1}{\rho_{\max }-\rho_{\min }} \int_{\rho_{\min }}^{\rho_{\max }}\left\langle\left|\frac{\Psi^{(k)}(\rho)}{\Psi_{\text {ref }}}\right|^{2}\right\rangle d \rho\right)+D T\right]$

Example measurements of sensing range are shown in Figure $\mathrm{H}-1$, where a $20 \%$ reduction is observed after nautical sunset.
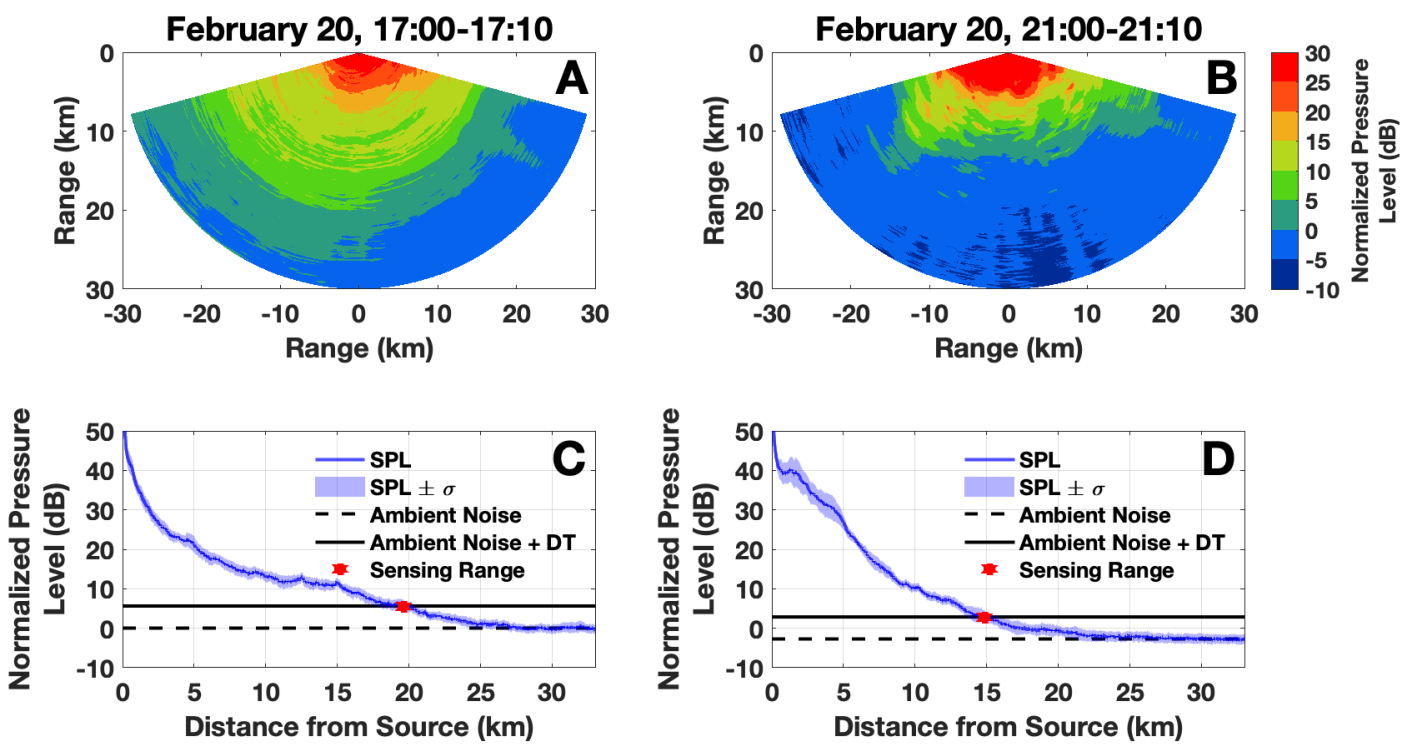

Figure H-1: Reductions in signal intensity and ambient noise caused by attenuation from herring groups resulted in a $20 \%$ reduction in sensing range after nautical sunset. "Sensing range" is defined here as the range at which scattered returns from the environment can be observed above ambient noise. OAWRS maps of normalized pressure level are generated by averaging 12 instantaneous OAWRS images at $955 \mathrm{~Hz}$ at 17:00-17:10 (A) and 21:00-21:00 (B). The mean sensing range over each hour-long interval is calculated by averaging each sound pressure level map across all azimuthal angles, excluding angles within $25^{\circ}$ of endfire (C-D). Ambient noise (black dotted lines in C-D) is measured for each time interval as the average sound pressure level above $29.5 \mathrm{~km}$, where sound pressure level is flat enough to be considered dominated by ambient noise (Equation A4). Sensing range (red dots in C-D) is measured as the range where sound pressure level (blue lines in C-D) falls within the detection threshold $D T=5.6 \mathrm{~dB}$ of the ambient noise. Over the course of the four hours shown here, sensing range reduces by $20 \%$. Light blue patches in C-D denote the standard deviation of normalized pressure level. The pressure levels shown here are normalized by the ambient noise level measured between 17:00 and 17:10 (A and B). 


\section{Appendix I}

\section{Correcting OAWRS transmissions for attenuation from fish}

Here a method is introduced for correcting OAWRS transmissions for attenuation from fish using a theoretical formulation that has been previously shown to be consistent with experimental measurements of attenuation in a waveguide environment (Appendix D) [43, 15]. The theoretical decay due to attenuation depends on the average population density of fish within the sensing region $\bar{n}_{A}$, which is estimated by modeling fish-attenuated scattering strength $\left(\widetilde{S S} \equiv S S-\triangle S P L_{2 w a y}\right)$ assuming a horizontally uniform distribution of fish within the OAWRS sensing region and performing a least-squares fit between measured and modeled fish-attenuated scattering strength. Measurements of mean fish-attenuated scattering

strength $\left(\left\langle\widetilde{S S}_{\text {data }}\right\rangle\right)$ with respect to range $\rho$ are calculated by correcting OAWRS sound pressure level maps for source level, areal resolution footprint, spreading loss and seafloor attenuation according to Equation A4 and then averaging across azimuthal angle excluding beams within $25^{\circ}$ of endfire. Modeled fish-attenuated scattering strength $\left(\widetilde{S S}_{\text {model }}\right)$ at range $\rho$ and assuming average areal density $\bar{n}_{A}$ is given by:

$$
\widetilde{S S}_{\text {model }}\left(\bar{n}_{A}, \rho\right) \equiv 10 \log _{10}\left(10^{S S_{\text {fish }}\left(n_{A}\right) / 10}+10^{S S_{\text {seafloor }} / 10}\right)-\Delta S P L_{2 w a y, \text { model }}\left(n_{A}, \rho\right)
$$

where $S S_{\text {seafloor }}=-43 \mathrm{~dB}$ is the measured scattering strength of seafloor in the region and $S S_{f i s h}$ is the scattering strength of the fish groups, given by:

$$
S S_{f i s h}\left(n_{A}\right)=T S+10 \log _{10}\left(n_{A}\right)
$$


where $T S$ is the target strength of an individual fish (Appendix C) and $\triangle S P L_{2 \text { way,model }}$ is the modeled reduction in sound pressure level caused by attenuation from fish (Equation 2.11).

The average areal density of the herring within the sensing region of the OAWRS system is determined using a least-square estimation performed by maximizing the likelihood function. Since the acoustic field can be described as a circular complex Gaussian random variable (CCGR) and the time-bandwidth product of the acoustic measurements $\mu=(1$ second $)(50 \mathrm{~Hz}) \gg 1$, intensity measurements in the logarithmic domain such as fishattenuated scattering strength can be well-approximated as Gaussian random variables with variance independent of the mean $[33,34]$. The mean herring areal density is estimated by maximizing the log-likelihood function $\ell\left(\bar{n}_{A}\right)$ assuming that fish-attenuated scattering strength $\widetilde{S S}$ is a Gaussian random variable with variance independent of the mean:

$$
\ell\left(\bar{n}_{A}\right)=\int_{\rho_{\text {min }}}^{\rho_{\text {sens }}}-\frac{\left(\widetilde{S S}_{\text {model }}\left(\bar{n}_{A}, \rho\right)-\left\langle\widetilde{S S}_{\text {data }}(\rho)\right\rangle\right)^{2}}{\sigma_{\widetilde{S S}}(\rho)^{2}} \rho d \rho
$$

where $\sigma_{\widetilde{S S}}(\rho)^{2}$ is the variance of $\widetilde{S S}_{d a t a}$ in $\mathrm{dB}$ at range $\rho$, and the differential $\rho d \rho$ is chosen in order to integrate over range in polar coordinates. The minimum range $\rho_{\text {min }}=2 \mathrm{~km}$ is chosen so that acoustic data is only considered at ranges well above farfield $\rho>L^{2} / \lambda$, where $L=47.25$ is the length of the receiver array [57] and $\lambda=1.57 \mathrm{~m}$ is the acoustic wavelength at the sensing frequency $955 \mathrm{~Hz}$. The sensing range $\rho_{\text {sens }}$ is defined in Section K. Once the average areal density $\bar{n}_{A}$ is determined for a given OAWRS transmission, a range-dependent correction can be applied to scattering strength maps at each azimuthal angle according to:

$$
S S(\rho, \theta)=\widetilde{S S}_{\text {data }}(\rho, \theta)+\Delta S P L_{2 w a y}\left(\bar{n}_{A}, \rho\right)
$$




\section{Appendix J}

\section{The Effect of Attenuation from Fish on Ambient Noise}

Here a formulation is introduced for predicting reductions in ambient noise levels due to attenuation from fish. This formulation is found to be consistent with observed reductions in ambient noise when fish groups congregate around the OAWRS system at nautical sunset (Figure 3-7). Since the dominant source of ambient noise is assumed to be surface waves, ambient noise is modeled as coming from a uniform set of uncorrelated monopoles at the surface. The intensity of the ambient noise field in a shallow water environment is then given [30] by:

$$
I=\frac{8 \pi^{2} q^{2}}{k^{2}} \int_{0}^{\infty} \xi\left|g\left(\xi ; z, z_{0}\right)\right| d \xi
$$

where $k$ is the wavenumber, $q$ is the amplitude of an individual monopole at depth $z_{0}$ near the surface, and $g\left(\xi ; z, z_{0}\right)$ is the wavenumber-depth Green function for horizontal wavenumber $\xi$ and receiver depth $z$, which can be written in a waveguide environment in terms of normal modes [30] as follows:

$$
g\left(\xi ; z, z_{0}\right)=\frac{d}{2 \pi} \sum_{n} \frac{u_{n}(z) u_{n}\left(z_{0}\right)}{\xi^{2}-\xi_{n}^{2}}
$$

where $\xi_{n}$ is the modal horizontal wavenumber, $d$ is water density, and the amplitude of mode $n$ at receiver depth $z$ is given by $u_{n}(z)$.

In order for the integral in Equation A1 to converge, some attenuation must be included in the system. This is because the signal from each monopole suffers cylindrical spreading loss, but the energy radiated by the monopoles increases with the range from the receiver squared [30]. Environmental attenuation (not including attenuation from fish) is introduced 
by letting the wavenumber $k$ be complex:

$$
k=\frac{2 \pi f}{c}+i \epsilon
$$

where $f$ is the frequency, $c$ is the speed of sound in water, and $\epsilon$ is a coefficient for environmental attenuation excluding fish $[30,1]$. The modal wavenumbers $\left(\xi_{n}\right)$ are also assumed to be complex, with the form:

$$
\xi_{n}=\kappa_{n}+i \alpha_{n}
$$

where the modal attenuation coefficient $\alpha_{n}$ is given [24] by

$$
\alpha_{n}=\frac{\epsilon}{\kappa_{n}} \int_{0}^{D} \frac{2 \pi f}{c(z)}\left|u_{n}(z)\right|^{2} d z
$$

where $D$ is the water depth. In the absence of attenuation from fish, the ambient field can then be written [30] as:

$$
I=\frac{\pi q^{2} d^{2}}{2 k^{2}} \sum_{n} \frac{u_{n}(z)^{2} u_{n}\left(z_{0}\right)^{2}}{\kappa_{n} \alpha_{n}}
$$

In the presence of fish, the modal wavenumber equation is modified to include the modal coefficients for attenuation and dispersion from fish $\left(\nu_{n}\right)$ :

$$
\xi_{n}=\kappa_{n}+i \alpha_{n}+\nu_{n}=\left(\kappa_{n}+\Re\left[\nu_{n}\right]\right)+i\left(\alpha_{n}+\Im\left[\nu_{n}\right]\right)
$$

A general formulation for $\nu_{n}$ can be found in Equation (60a) of [43]. Since long-range ocean sensing systems typically operate at low frequencies where the acoustic wavelength is larger than the dimensions of a fish, individual fish will be compact scatterers and the dispersion and attenuation coefficients for fish shoals can be obtained from Equation 2.5. Equations for the scatter function $S$ are shown in Appendix C. If the fish are assumed to be uniformly ditributed in depth with mean shoal depth $z_{m}$ and vertical thickness $H$, volume density can be written as $n_{A} / H$ and the dispersion and attenuation coefficients can be written as a function of $n_{A}$ :

$$
\nu_{n}\left(n_{A}\right)=\int_{z_{m}-H / 2}^{z_{m}+H / 2} \frac{2 \pi}{k} \frac{1}{\xi_{n}} \frac{1}{d}\left(u_{n}\left(z_{t}\right)\right)^{2} \frac{n_{A}}{H}\left\langle S\left(z_{t}\right)\right\rangle d z_{t}
$$

Following the same derivation that led to Equation A6, the ambient noise field in the 
presence of attenuation from fish can be written as:

$$
I_{a t t n}\left(n_{A}\right)=\frac{\pi q^{2} d^{2}}{2 k^{2}} \sum_{n} \frac{u_{n}(z)^{2} u_{n}\left(z_{0}\right)^{2}}{\left(\kappa_{n}+\Re\left[\nu_{n}\left(n_{A}\right)\right]\right)\left(\alpha_{n}+\Im\left[\nu_{n}\left(n_{A}\right)\right]\right)}
$$

The decrease in ambient noise level from attenuation as fish density increases from $n_{A 1}$ to $n_{A 2}$ can then be given by:

$$
\begin{aligned}
\Delta N L_{\text {partial }}\left(n_{A 1}, n_{A 2}\right)=10 & \log _{10}\left(\frac{I_{\text {attn }}\left(n_{A 1}\right)}{I_{\text {attn }}\left(n_{A 2}\right)}\right) \\
=10 \log _{10}[ & \left(\sum_{n} \frac{u_{n}(z)^{2} u_{n}\left(z_{0}\right)^{2}}{\left(\kappa_{n}+\Re\left[\nu_{n}\left(n_{A 1}\right)\right]\right)\left(\alpha_{n}+\Im\left[\nu_{n}\left(n_{A 1}\right)\right]\right)}\right) \\
& \left.\left(\sum_{n} \frac{u_{n}(z)^{2} u_{n}\left(z_{0}\right)^{2}}{\left(\kappa_{n}+\Re\left[\nu_{n}\left(n_{A 2}\right)\right]\right)\left(\alpha_{n}+\Im\left[\nu_{n}\left(n_{A 2}\right)\right]\right)}\right)^{-1}\right]
\end{aligned}
$$

where $n_{A 1}<n_{A 2}$.

The decrease in ambient noise due to the formation of herring groups at nautical sunset on February 20, 2014 is predicted using Equation A10. The average areal density after nautical sunset $\left(n_{A 1}\right)$ is determined to be $0.08 \mathrm{fish} / \mathrm{m}^{2}$ by taking the average of OAWRS areal density measurements between 17:00 and 18:30 (Figure 3-4D). The average areal density after nautical sunset $\left(n_{A 2}\right)$ is determined to be $0.2 \mathrm{fish} / \mathrm{m}^{2}$ by taking the average of OAWRS measurements of areal density between 19:30 and 21:00 (Figure 3-4D). The modeled coefficient for environmental attenuation excluding fish $\epsilon$ is determined by maximizing the following likelihood function:

$$
\ell(\epsilon)=\sum_{i=1}^{N_{f}}\left(\frac{-\left(N L_{\text {model }}\left(\epsilon, f_{i}\right)-\left\langle\Delta N L\left(f_{i}\right)\right\rangle\right)}{\sigma_{\Delta N L}\left(f_{i}\right)^{2}}\right)
$$

where $\left\langle\Delta N L\left(f_{i}\right)\right\rangle$ is the mean reduction in ambient noise at frequency $f_{i}, \sigma_{\Delta N L}\left(f_{i}\right)$ is the standard deviation at frequency $f_{i}, N_{f}$ is the number of frequencies where $\Delta N L$ is measured, and the coefficient for environmental attenuation excluding fish $\epsilon$ is assumed to be in units of $\lambda^{-1}$. The likelihood function is maximized at $\epsilon=5 \times 10^{-5} \lambda^{-1}$, which is in the same order of magnitude as previous experimental measurements of environmental attenuation in waveguide environments [1]. 


\section{Appendix K}

\section{Predicting Sensing Range in the Presence of Attenuation from Fish}

Here a formulation is introduced for predicting sensing range in the presence of attenuation from fish. As in Equation A1, sensing range $\left(\rho_{\text {sens }}\right)$ is modeled as:

$$
\rho_{\text {sens }}\left(f, n_{A}\right)=\min \left[\rho \mid S P L_{A}\left(\rho, f, n_{A}\right)>N L(f)-\Delta N L\left(f, n_{A}\right)+D T\right]
$$

where $S P L_{A}$ is the the azimuthally-intensity-averaged-sound pressure level of the received signal, $N L$ is the ambient noise level, $D T$ is the detection threshold, $f$ is the sensing frequency, and it is assumed that fish are uniformly distributed in the horizontal within the sensing region with average areal density $n_{A}$. Ambient noise level $N L$ is determined from measurements before nautical sunset (17:00-18:30 on February 20) in order to avoid attenuation effects caused by the formation of herring groups after nautical sunset (Figure 3-7A). The reduction in ambient noise due to attenuation from fish $(\Delta N L)$ is modeled according to Appendix J. As in Equation A2, the azimuthally-intensity-averaged-sound pressure level $S P L_{A}$ is estimated by averaging the intensity of the scattered field across azimuthal angle $\theta$ :

$$
S P L_{A}\left(\rho, f, n_{A}\right)=10 \log _{10}\left(\frac{1}{\theta_{\max }-\theta_{\min }} \int_{\theta_{\min }}^{\theta_{\max }}\left|\frac{\Psi\left(\rho, f, n_{A}, \theta\right)}{\Psi_{\text {ref }}}\right|^{2} d \theta\right)
$$

where $\Psi\left(\rho, f, n_{A}, \theta\right)$ is the modeled scattered field, $\Psi_{r e f}=1 \mu \mathrm{Pa}$ is the reference pressure for underwater sound, and the limits of integration $\theta_{\min }$ and $\theta_{\max }$ are chosen to exclude beams within $25^{\circ}$ of endfire. Assuming that scattered returns from the environment are dominated by scattering from fish groups rather than seafloor scattering, the intensity of the scattered 
field $\left|\frac{\Psi\left(\rho, f, n_{A}, \theta\right)}{\Psi_{\text {ref }}}\right|^{2}$ is modeled according [15] to

$10 \log _{10}\left|\frac{\Psi\left(\rho, f, n_{A}, \theta\right)}{\Psi_{\text {ref }}}\right|^{2}=S L+T L A(\rho, f, \theta)+T S(f)+10 \log _{10}\left(n_{A}\right)-\Delta S P L_{2 w a y}\left(\rho, f, n_{A}\right)$

Transmission loss area TLA is calculated according to Equation A2 assuming constant water depth based on bathymetric measurements in the region studied (Table 2.1). The formulation for target strength $T S$ is shown in Appendix $\mathrm{C}$ and the formulation for the decrease in sound pressure level during two-way propagation due to attenuation from fish $\left(\triangle S P L_{2 w a y}\right)$ is shown in Appendix D of [15]. Both target strength and attenuation from fish depend on species-specific parameters shown in Table 2.1. 


\section{Appendix L}

\section{Synoptic Echosounder Measurements of Herring Areal Density and Depth Distribution}

Synoptic echosounder measurements of Ålesund herring population density collected during the 2014 OAWRS experiment (Figure L-2) are consistent with estimates of herring population shown in Figure 3-4. Shoal volume density $\left(\mathrm{fish} / \mathrm{m}^{3}\right)$ is measured from echogram data according [19] to

$$
n_{V}=\frac{1}{\sigma_{b s}} s_{v}
$$

where $s_{v}$ is the volume backscattering coefficient $\left(\mathrm{m}^{-1}\right)$ and $\sigma_{b s}=10^{T S_{C F F S} / 10}$ is the fish backscattering cross section of an individual fish at at the echosounder sensing frequency (38 kHz) in units of $\mathrm{m}^{2}$, where $T S_{C F F S}$ is the target strength at this frequency, given [41] by

$$
T S_{C F F S}=20 \log _{10} L_{T L}-2.3 \log _{10}(1+z / 10)-65.4
$$

where $L_{T L}$ is the length of an individual herring $(\mathrm{cm})$, and $z$ is the water depth $(\mathrm{m})$. Shoal areal density $\left(\right.$ fish $/ \mathrm{m}^{2}$ ) is given by

$$
n_{A}=\int_{z_{1}}^{z_{2}} n_{V} d z
$$

where $z_{1}$ and $z_{2}$ delimit the depth bounds of the fish aggregations.

The probability density function of herring depth $p(z)$ is measured by aggregating echosounder measurements of herring in Ålesund waters according to:

$$
p(z)=\frac{\int_{\rho} n_{V, C F F S}(\rho, z) d \rho}{\int_{z} \int_{\rho} n_{V, C F F S}(\rho, z) d \rho d z}
$$


where $n_{V, C F F S}(\rho, z)$ is the volumetric population density of herring at horizontal position $\rho$ and depth $z$ measured by echosounders between 17:00 and 21:00 on February 20, where $p(z)$ appears in Figure L-3.
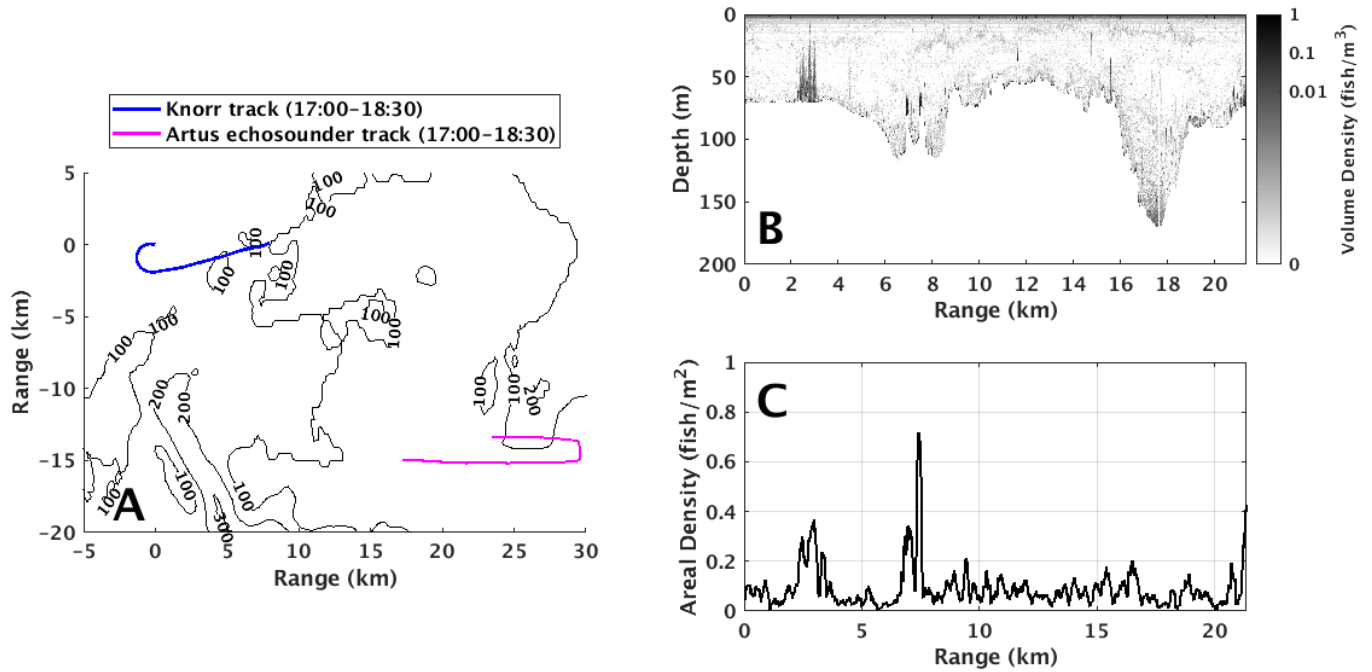

Figure L-1: Synoptic echosounder measurements of Ålesund herring shoals are consistent with OAWRS population density measurements before nautical sunset. In (A), bathymetric contours are shown in black, the path of the research vessel towing the OAWRS system (RV Knorr) between 17:00 and 18:30 on February 20, 2014 is overlain in blue, and the path of the research vessel from which echosounder measurements were recorded during this time is overlain in magenta. Echosounder measurements of volumetric population density are shown in (B), and measurements of areal population density are shown in (C). Sparse herring groups are observed with population densities on the order of $0.3 \mathrm{fish} / \mathrm{m}^{2}$, which is consistent with OAWRS population density measurements during this time (Figure 3-2). 

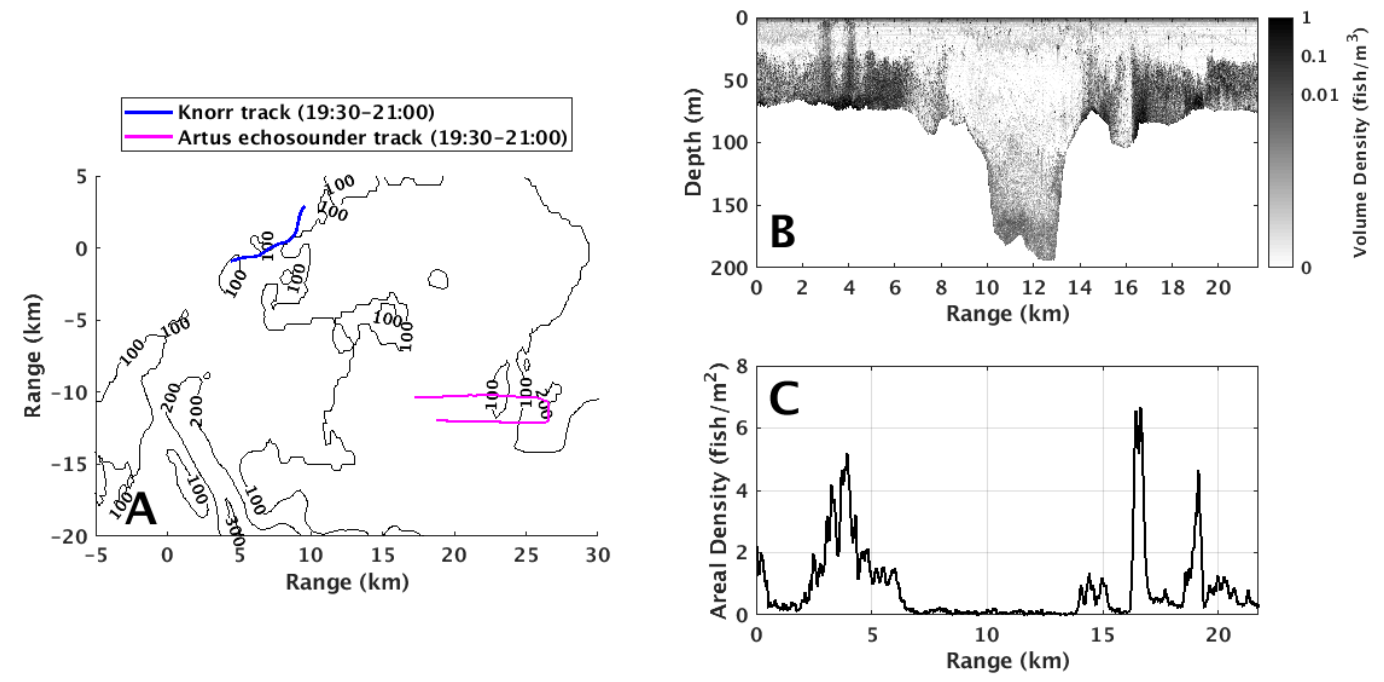

Figure L-2: Synoptic echosounder measurements of Ålesund herring shoals are consistent with OAWRS population density measurements after nautical sunset. In (A), bathymetric contours are shown in black, the path of the research vessel towing the OAWRS system (RV Knorr) between 19:30-21:00 on February 20, 2014 is overlain in blue, and the path of the research vessel from which echosounder measurements were recorded during this time is overlain in magenta. Echosounder measurements of volumetric population density are shown in (B), and measurements of areal population density are shown in (C). Several dense herring groups are observed with population densities on the order of $5 \mathrm{fish} / \mathrm{m}^{2}$, which is consistent with OAWRS population density measurements during this time (Figure 3-5).

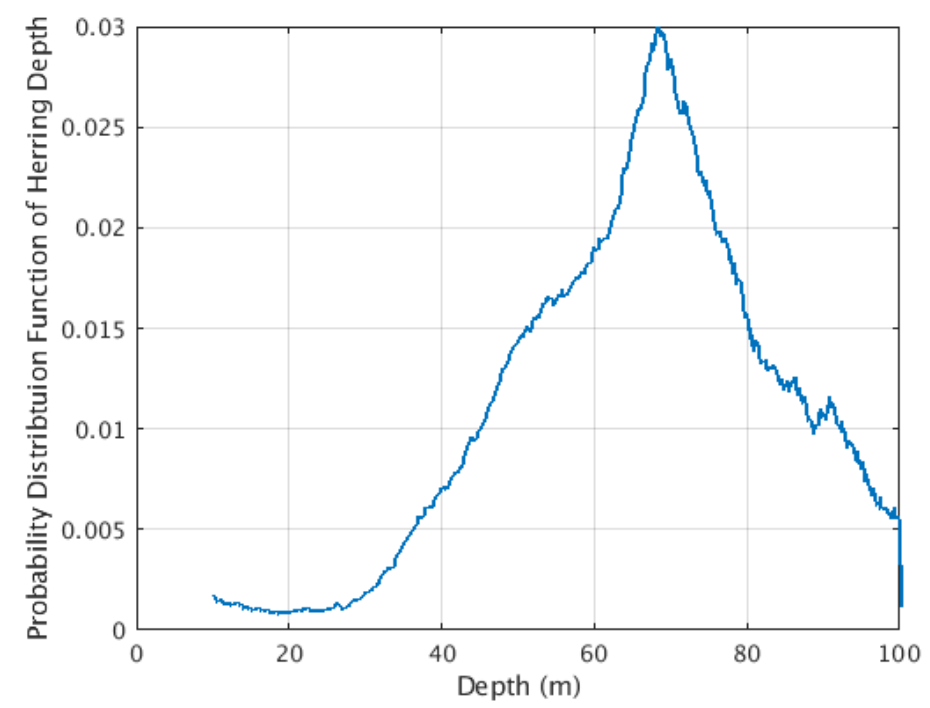

Figure L-3: Probability density function of herring depth $p(z)$ inverted from echosounder measurements on 2014 February 20 between 17:00 and 21:00 in Ålesund spawning grounds (Equation A4). 


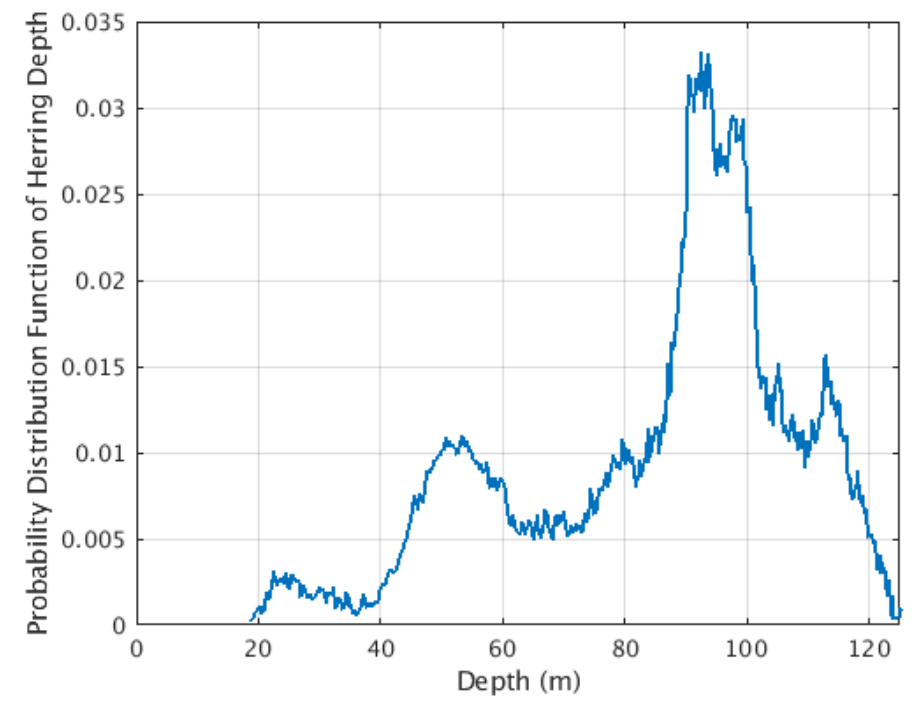

Figure L-4: Probability density function of herring depth $p(z)$ inverted from echosounder measurements on 2014 February 21 between 3:10 and 6:00 in Alesund spawning grounds (Equation A4). 


\section{L.1 Measurements of herring population from OAWRS pop- ulation density maps}

Herring population within OAWRS survey regions is calculated by integrating OAWRS measurements of population density over regions where the population density is greater than $0.2 \mathrm{fish} / \mathrm{m}^{2}$, which is the critical population density at which large herring shoals were found to form $[37,38]$. It is found that selecting regions above this threshold is an effective way to include discrete herring shoals in population measurements without including measurements contaminated by background seafloor scattering (Figure L-1). It is found that $87 \%$ of the herring population measured by echosounders during this experiment was above the $0.2 \mathrm{fish} / \mathrm{m}^{2}$ threshold (Figure L-2).

Figure L-1 (facing page): Echosounder measurements confirm that the majority of herring contained in the discrete shoals observed by OAWRS are in regions where the population density is greater than the critical population density $0.2 \mathrm{fish} / \mathrm{m}^{2}[37,38]$. An OAWRS population denisty map from February 21, 3:26:39 is shown in (A), where the path of an echosounder is overlain in white and the corresponding echosounder data from 2:48-3:40 are shown in (B). The green dot in (A) and the green line in (B) correspond to the echosounder position for the OAWRS image shown in (A). Black dotted lines in (B) designate regions $\alpha, \beta$, and $\gamma$, where both systems co-register dense fish groups (A). The areal population density measured by the echosounder along this transect is shown in $(\mathrm{C})$, where red data denotes regions where the OAWRS population density is greater than $0.2 \mathrm{fish} / \mathrm{m}^{2}$ and black data denotes regions where the OAWRS population density is less than $0.2 \mathrm{fish} / \mathrm{m}^{2}$. It is found that selecting regions above the $0.2 \mathrm{fish} / \mathrm{m}^{2}$ threshold is an effective way to segment discrete herring shoals (red data in C) without including measurements potentially affected by scintillation and contamination from background seafloor scattering (black data in $\mathrm{C}$ ). By contrast, a significant portion of OAWRS data above $0.05 \mathrm{fish} / \mathrm{m}^{2}$ falls outside the dense, discrete shoal (D). 

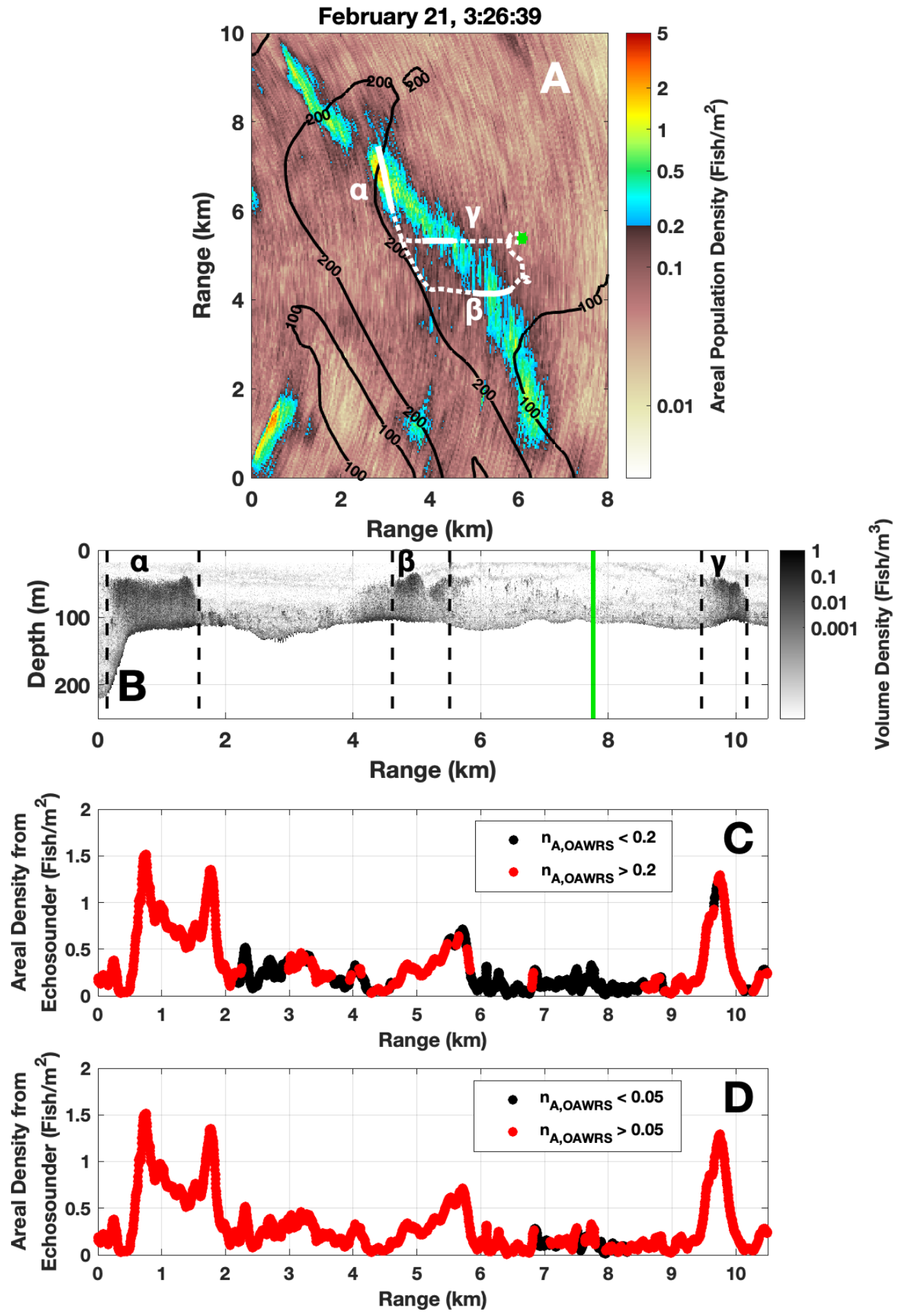


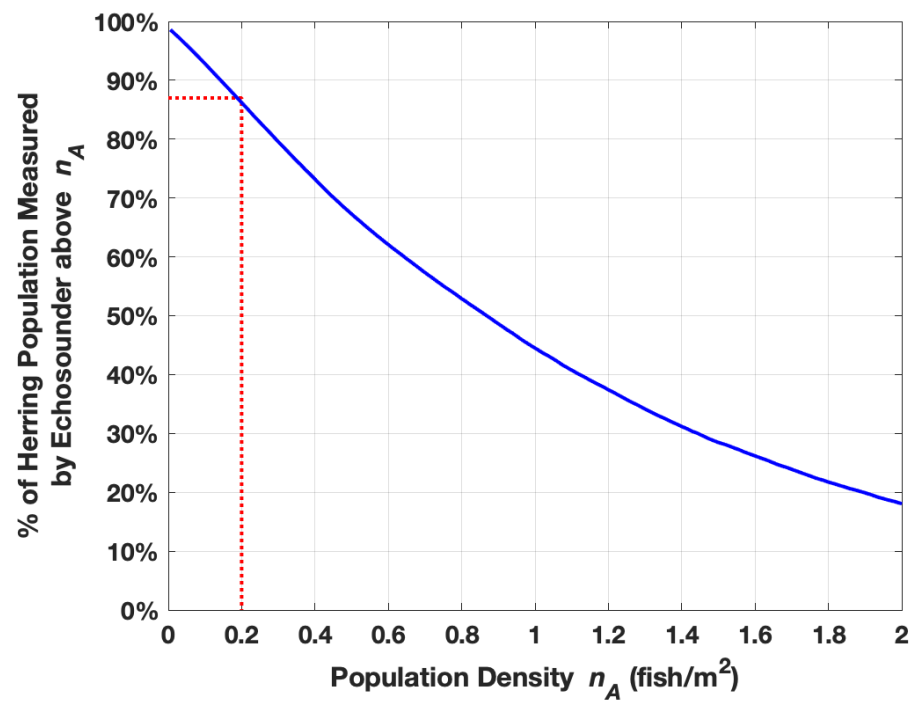

Figure L-2: The percentage of the herring population measured by echosounders between February 17, 23:30 and February 21, 21:00 above the population density threshold $n_{A}$ is shown here. $87 \%$ of the herring population is found to be above the critical population density $0.2 \mathrm{fish} / \mathrm{m}^{2}$ (red dotted line). 


\section{Appendix M}

\section{Measured wind speed variations}

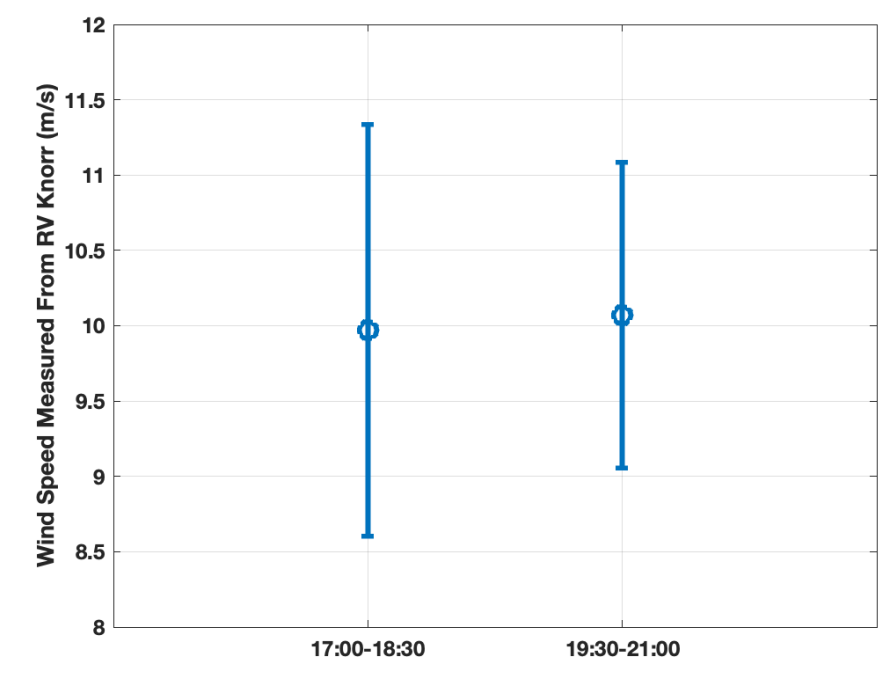

Figure M-1: Wind speed measurements recorded by the research vessel towing the OAWRS system (RV Knorr) are shown here from February 20, 2014 between 17:00-18:30 (before nautical sunset) and between 19:30-21:00 (after nautical sunset). There is no statistically significant difference between wind speed before and after sunset, indicating that reductions in ambient noise after sunset are not caused by changes in wind speed.

Wind speed measurements recorded by the research vessel towing the OAWRS system (RV Knorr) before and after nautical sunset on February 20, 2014 are shown in Figure M-1. There is no statistically significant difference between wind speed before and after sunset, which demonstrates that reductions in ambient noise after sunset are not caused by changes in wind speed. 


\section{Appendix N}

\section{Detecting and Measuring the Received Level of Ship-Radiated Tonals}

Measurements of received level beamformed in the direction of the Artus are classified as "Artus detected" or "Artus not detected" depending on whether the beamformed received level is greater or less than the noise level $N L$ corrected for the array gain $A G$ plus the detection threshold $D T$, according to:

$$
\begin{cases}R L>N L-A G+D T & \text { Artus detected } \\ R L<N L-A G+D T & \text { Artus not detected }\end{cases}
$$

The noise level corrected for the array gain $(N L-A G)$ is measured as the average received level beamformed in the direction of the Artus at frequencies between $1620 \mathrm{~Hz}$ and 1650 $\mathrm{Hz}$, where no significant tonals contaminate measurements during the time studied. These measurements of $N L-A G$ may include broadband noise from the Artus and other ships (not including tonals) or other sources of ambient noise. The detection threshold $D T=$ $1.5 \mathrm{~dB}$ is calculated as the standard deviation of the Artus tonal at frequencies between $f_{c} \pm B / 2$ (Figure 4-2), where $f_{c}=1584 \mathrm{~Hz}$ is the center frequency of the Artus tonal and $B=30 \mathrm{~Hz}$ is the $3 \mathrm{~dB}$-down bandwidth of the Artus tonal averaged over 30 seconds. Since the signal from the Artus is cylostationary rather than a pure tonal [22], standard deviation measurements are not in agreement with the theoretically-derived standard deviation of a pure tonal after saturated multipath propagation derived in [33, 34]. 


\section{Appendix $\mathrm{O}$}

\section{Fluctuations in the Speed of the Artus}

Variations in the speed of the Artus cannot fully explain observed reductions in the intensity of the received signal of the Artus (Figure 4-2), demonstrating that attenuation from fish is a significant factor leading to intensity reductions and detection losses. With the exception of the ship turns at 3:10,4:15, and 5:20, variations in the speed of the Artus are smaller than $0.5 \mathrm{~m} / \mathrm{s}$. 


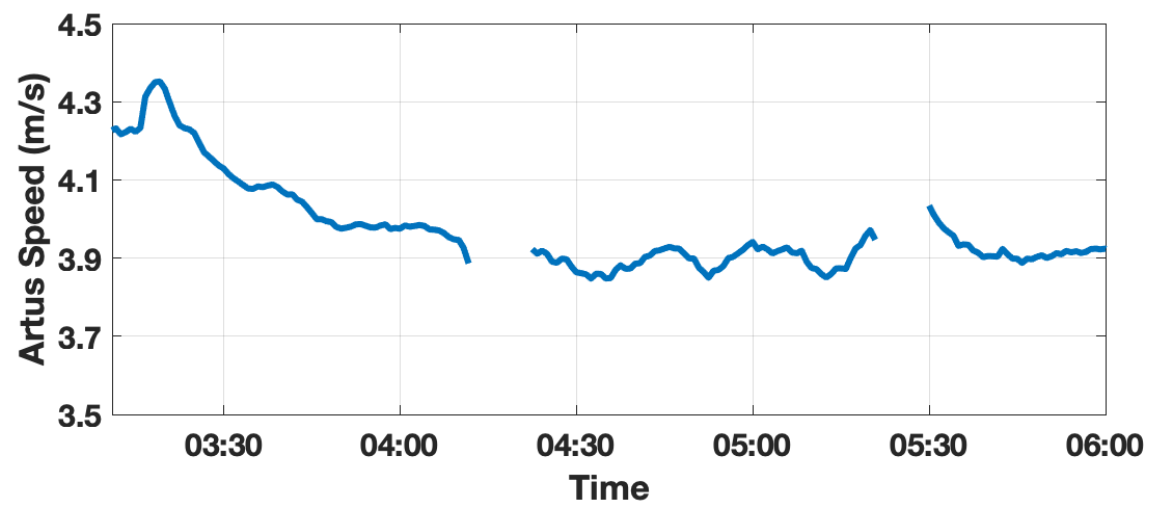

Figure O-1: The speed of the Artus between 3:10 and 6:00 on February 21, 2014 is shown here. With the exception of the ship turns at 4:09-4:25, and 5:18-5:33, variations in the speed of the Artus are smaller than $0.5 \mathrm{~m} / \mathrm{s}$. 


\section{Bibliography}

[1] M. Andrews, T. Chen, and P. Ratilal. Empirical dependence of acoustic transmission scintillation statistics on bandwidth, frequency, and range in new jersey continental shelf. J. Acoust. Soc. Am., 125:111-124, 2009.

[2] M. Andrews, Z. Gong, and P. Ratilal. Effects of multiple scattering, attenuation, and dispersion in waveguide sensing of fish. J. Acoust. Soc. Am., 130:1253-1271, 2011.

[3] A. R. Appenzeller and W. C. Leggett. Bias in hydroacoustic estimates of fish abundance due to acoustic shadowing: evidence from day-night surveys of vertically migrating fish. Can. J. Fish. Aquat. Sci., 49:2179-2189, 1992.

[4] T. Chen, P. Ratilal, and N. C. Makris. Mean and variance of the forward field propagated through three-dimensional random internal waves in a continental-shelf waveguide. J. Acoust. Soc. Am., 118:3560-3574, 2005.

[5] T. Chen, P. Ratilal, and N. C. Makris. Temporal coherence after multiple forward scattering through random three-dimensional inhomogeneities in an ocean waveguide. J. Acoust. Soc. Am., 124:2812-2822, 2008.

[6] Tianrun Chen. Mean, variance, and temporal coherence of the $3 d$ acoustic field forward propagated through random inhomogeneities in continental-shelf and deep ocean waveguides. PhD thesis, Massachusetts Institute of Technology, Cambridge, MA, 2009.

[7] P. Ching and D. Weston. Wideband studies of shallow-water acoustic attenuation due to fish. J. Sound. Vib., 18:499-510, 1971.

[8] B. Cho and N. C. Makris. Predicting the effects of random ocean dynamic processes on underwater acoustic sensing and communication. Scientific Reports, 10:4525, 2020.

[9] M. Collins. A split-step padé solution for the parabolic equation method. J. Acoust. Soc. Am., 93:1736-1742, 1993.

[10] A. Berdahl P. A. Westley S. A. Levin I. D. Couzin and T. P. Quinn. A collective navigation hypothesis for homeward migration in anadromous salmonids. Fish and Fisheries, 17:525-542, 2016.

[11] I. E. Davies. Attenuation of sound by schooled anchovies. J. Acoust. Soc. Am., 54:213217, 1973.

[12] O. Diachok. Effects of absorptivity due to fish on transmission loss in shallow water. J. Acoust. Soc. Am., 105:2107-2128, 1999. 
[13] O. Diachok, B. Liorzou, and C. Scalabrin. Estimation of the number density of fish from resonance absorptivity and echo sounder data. ICES J. Mar. Sci., 58:137-153, 2001.

[14] O. Diachok and S. Wales. Concurrent inversion of geo- and bio-acoustic parameters from transmission loss measurements in the yellow sea. J. Acoust. Soc. Am., 128:1965-1976, 2005 .

[15] D. Duane, B. Cho, A. D. Jain, O. R. Godø, and N. C. Makris. The effect of attenuation from fish shoals on long-range, wide-area acoustic sensing. Remote Sens., 11:2464, 2019.

[16] I. Dyer. Statistics of sound propagation in the ocean. J. Acoust. Soc. Am., 48:337-345, 1970.

[17] K. Foote. Correcting acoustic measurements of scatter density for extinction. J. Acoust. Soc. Am., 88:1543-1546, 1990.

[18] H. A. Garcia, C. Zhu, M. E. Schinault, A. I Kaplan, N. O. Handegard, O. R. God $\tilde{A} \ddot{y}$, H. Ahonen, N. C. Makris, D. Wang, W. Huang, and P. Ratilal. TemporalâĂŞspatial, spectral, and source level distributions of fin whale vocalizations in the norwegian sea observed with a coherent hydrophone array. ICES J. Mar. Sci., 76:268-283, 2019.

[19] Z. Gong, M. Andrews, S. Jagannathan, R. Patel, J. M. Jech, N. C. Makris, and P. Ratilal. Low-frequency target strength and abundance of shoaling atlantic herring (Clupea harengus) in the gulf of maine during the ocean acoustic waveguide remote sensing 2006 experiment. J. Acoust. Soc. Am., 127:104-123, 2010.

[20] Z. Gong, T. Chen, P. Ratilal, and N. C. Makris. Temporal coherence of the acoustic field forward propagated through a continental shelf with random internal waves. $J$. Acoust. Soc. Am., 134:3476-3485, 2013.

[21] Z. Gong, A. D. Jain, D. H. Yi, F. Wu, A. Zorn, P. Ratilal, and N. C. Makris. Ecosystem scale acoustic sensing reveals humpback whale behavior synchronous with herring spawning processes and re-evaluation finds no effect of sonar on humpback song occurence in the gulf of maine in fall 2006. Plos One, 9:1930-1936, 2014.

[22] W. Huang, D. Wang, H. Garcia, O. R. Godø, and P. Ratilal. Continental shelf-scale passive acoustic detection and characterization of diesel-electric ships using a coherent hydrophone array. Remote Sens., 9:772, 2017.

[23] W. Huang, D. Wang, and P. Ratilal. Diel and spatial dependence of humpback song and non-song vocalizations in fish spawning ground. Remote Sens., 8:712, 2016.

[24] F. Ingenito. Measurements of mode attenuation coefficients in shallow water. J. Acoust. Soc. Am., 53:858-863, 1973.

[25] F. Ingenito. Scattering from an object in a stratified medium. J. Acoust. Soc. Am., 82:2051-2059, 1987.

[26] S. Jagannathan, I. Bertsatos, D. Symonds, T. Chen, H. Nia, A. Jain, M. Andrews, Z. Gong, R. Nero, L. Ngor, M. Jech, O. R. God ̃̈̈̈, S. Lee, P. Ratilal, and N. C. Makris. Ocean acoustics waveguide remote sensing (oawrs) of marine ecosystems. Mar. Ecol. Prog. Ser., 395:137-160, 2009. 
[27] M. Johnson, P. T. Madsen, W. M. X. Zimmer, N. A. de Soto, and P. L. Tyack. Beaked whales echolocate on prey. Proc. Biol. Sci., 271:2239-2247, 2004.

[28] F. H. Jones and P. Scholes. Gas secretion and resorption in the swimbladder of the cod (Gadus morhua). J. Comp. Physiol., 10:108, 2018.

[29] V. Klemas. Fisheries applications of remote sensing: An overview. Fisheries Research, 148:124-136, 2013.

[30] W. A. Kuperman and F. Ingenito. Spatial correlation of surface generated noise in a stratified ocean. J. Acoust. Soc. Am., 67:1988-1996, 1980.

[31] R. Love. Resonant acoustic scattering by swimbladder-bearing fish. J. Acoust. Soc. Am., 64:571-580, 1978.

[32] R. Love, J. Fialkowski, and T. Jagielo. Target strength distributions of pacific sardine shoals: model results at $500 \mathrm{hz}$ to $10 \mathrm{khz}$. J. Acoust. Soc. Am., 137:539-555, 2016.

[33] N. C. Makris. A foundation for logarithmic measures of fluctuating intensity in pattern recognition. Optics Letters, 20(19):2012-2014, 1995.

[34] N. C. Makris. The effect of saturated transmission scintillation on ocean acoustic intensity measurements. J. Acoust. Soc. Am., 100:769-783, 1996.

[35] N. C. Makris, O. R. Godø, D. H. Yi, G. J. Macauley, A. D. Jain, B. Cho, Z. Gong, J. M. Jech, and P. Ratilal. Instantaneous areal population density estimation of entire atlantic cod and herring spawning groups and group size distribution relative to total spawning population. Fish and Fisheries, 20:1-13, 2018.

[36] N. C. Makris and P. Ratilal. A unified model for reverberation and submerged object scattering in a stratified ocean waveguide. J. Acoust. Soc. Am., 109:909-941, 2001.

[37] N. C. Makris, P. Ratilal, S. Jagannathan, Z. Gong, M. Andrews, I. Bertsatos, O. God Ã̈̈, R. Nero, and J. M. Jech. Critical population density triggers rapid formation of vast oceanic fish shoals. Science, 323:1734-1737, 2009.

[38] N. C. Makris, P. Ratilal, D. Symonds, S. Jagannathan, S. Lee, and R. Nero. Fish population and behavior revealed by instantaneous continental shelf-scale imaging. Science, 311:660-663, 2006.

[39] Marine Stewardship Council. International action needed on herring and blue whiting stocks . https://www.msc.org/media-centre/newsopinion/news/2020/12/04/international-action-needed-on-herring-and-blue-whitingstocks, 2020.

[40] H. Medwin and C. Clay. Acoustical Oceanography. John Wiley Sons Inc, New York, 1977.

[41] E. Ona. An expanded target-strength relationship for herring. ICES J. Mar. Sci., 60:493-499, 2003.

[42] X. F. Qiu, R. H. Zhang, W. H. Li, G. L. Jin, and B. X. Zhu. Frequency-selective attenuation of sound propagation and reverberation in the yellow sea. J. Sound. Vib., 220:331-342, 1999. 
[43] P. Ratilal and N. C. Makris. Mean and covariance of the forward field propagated through a stratified ocean waveguide with three-dimensional random inhomogeneities. J. Acoust. Soc. Am., 118:3532-3558, 2004.

[44] Lord Rayleigh. The Theory of Sound, volume 2. Dover, New York, 1896.

[45] Lord Rayleigh. On the transmission of light through an atmosphere containing small particles in suspension, and on the origin of the blue sky. Philos. Mag., 47:375-384, 1899.

[46] I. Røttingen. On the relation between echo intensity and fish density. FiskDir. Skr. Ser. HavUnders, 16:301-314, 1976.

[47] J. Simmonds and D. N. MacLennan. Fisheries Acoustics: Theory and Practice. WileyBlackwell, 2008.

[48] G. Skaret and A. Slotte. Herring submesoscale dynamics through a major spawning wave: duration, abundance fluctuation, distribution, and schooling. ICES J. Mar. Sci., 74:717-727, 2017.

[49] R. Torensen. Absorption of acoustic energy in dense herring schools studied by the attenuation in the bottom signal. Fisheries Research, 10:317-327, 1991.

[50] D. D. Tran, M. Andrews, and P. Ratilal. Probability distribution for energy of saturated broadband ocean acoustic transmission: Results from gulf of maine 2006 experiment. J. Acoust. Soc. Am., 132:3659-3672, 2012.

[51] D. D. Tran, W. Huang, A. C. Bohn, and D. Wang. Using a coherent hydrophone array for observing sperm whale range, classification, and shallow-water dive profiles. J. Acoust. Soc. Am., 135:3352-3363, 2014.

[52] United Nations. UN Report: NatureâĂŹs Dangerous Decline âĂŸUnprecedentedâĂŹ; Species Extinction Rates âĂŸAcceleratingâĂŹ. https://www.un.org/sustainabledevelopment/blog/2019/05/nature-declineunprecedented-report/, 2019.

[53] R. Urick. Principles of Underwater Sound. McGraw Hill, 1983.

[54] H. C. van de Hulst. Light Scattering by Small Particles. Dover, New York, 1957.

[55] D. Wang, H. Garcia, D. D. Tran, A. D. Jain, D. H. Yi, Z. Gong, J. M. Jech, Godø, N. C. Makris, and P. Ratilal. Vast assembly of vocal marine mammals from diverse species on fish spawning ground. Nature, 531:366-369, 2016.

[56] D. Wang, W. Huang, H. Garcia, and P. Ratilal. Vocalization source level distributions and pulse compression gains of diverse baleen whale species in the gulf of maine. Remote Sens., 8:881, 2016.

[57] D. Wang and P. Ratilal. Angular resolution enhancement provided by nonuniformlyspaced linear hydrophone arrays in ocean acoustic waveguide remote sensing. Remote Sens., 9:1036, 2017.

[58] G. M. Wenz. Acoustic ambient noise in the ocean: Spectra and sources. J. Acoust. Soc. Am., 34:1936âĂŞ-1956, 1962. 
[59] D. Weston, K. Stevens, J. Revie, and M. Pengelly. Multiple frequency studies of sound transmission fluctuations in shallow water. J. Sound. Vib., 18:487-497, 1971.

[60] D. E. Weston. Sound propagation in the presence of bladder fish. In V. M. Albers, editor, Underwater Acoustics, pages 55-88. Plenum Press, New York, 1967.

[61] D. H. Yi, Z. Gong, J. M. Jech, P. Ratilal, and N. C. Makris. Instantaneous 3d continental-shelf scale imaging of oceanic fish by multi-spectral resonance sensing reveals group behavior during spawning migration. Remote Sens., 10:108, 2018.

[62] D. H. Yi and N. C. Makris. Feasibility of acoustic remote sensing of large herring shoals and seafloor by baleen whales. Remote Sens., 8, 2016.

[63] X. Zhao and E. Ona. Estimation and compensation models for the shadowing effect in dense fish aggregations. ICES J. Mar. Sci., 60:155-163, 2002.

[64] C. Zhu, H. Garcia, A. Kaplan, M. Schinault, N. O. Handegard, O. R. Godø, W. Huang, and P. Ratilal. Detection, localization and classification of multiple mechanized ocean vessels over continental-shelf scale regions with passive ocean acoustic waveguide remote sensing. Remote Sens., 10:1699, 2018.

[65] C. Zhu, S. G. Seri, H. Mohebbi-Kalkhoran, and P. Ratilal. Long-range automatic detection, acoustic signature characterization and bearing-time estimation of multiple ships with coherent hydrophone array. Remote Sens., 12:3731, 2020. 